\title{
Imaging imagination : a multi-method investigation into the brain dynamics of visual mental imagery
}

Citation for published version (APA):

de Borst, A. W. (2011). Imaging imagination : a multi-method investigation into the brain dynamics of visual mental imagery. [Doctoral Thesis, Maastricht University]. Universiteit Maastricht. https://doi.org/10.26481/dis.20110520ab

Document status and date:

Published: 01/01/2011

DOI:

10.26481/dis.20110520ab

Document Version:

Publisher's PDF, also known as Version of record

\section{Please check the document version of this publication:}

- A submitted manuscript is the version of the article upon submission and before peer-review. There can be important differences between the submitted version and the official published version of record.

People interested in the research are advised to contact the author for the final version of the publication, or visit the DOI to the publisher's website.

- The final author version and the galley proof are versions of the publication after peer review.

- The final published version features the final layout of the paper including the volume, issue and page numbers.

Link to publication

\footnotetext{
General rights rights.

- You may freely distribute the URL identifying the publication in the public portal. please follow below link for the End User Agreement:

www.umlib.nl/taverne-license

Take down policy

If you believe that this document breaches copyright please contact us at:

repository@maastrichtuniversity.nl

providing details and we will investigate your claim.
}

Copyright and moral rights for the publications made accessible in the public portal are retained by the authors and/or other copyright owners and it is a condition of accessing publications that users recognise and abide by the legal requirements associated with these

- Users may download and print one copy of any publication from the public portal for the purpose of private study or research.

- You may not further distribute the material or use it for any profit-making activity or commercial gain

If the publication is distributed under the terms of Article $25 \mathrm{fa}$ of the Dutch Copyright Act, indicated by the "Taverne" license above, 


\title{
Imaging Imagination
}

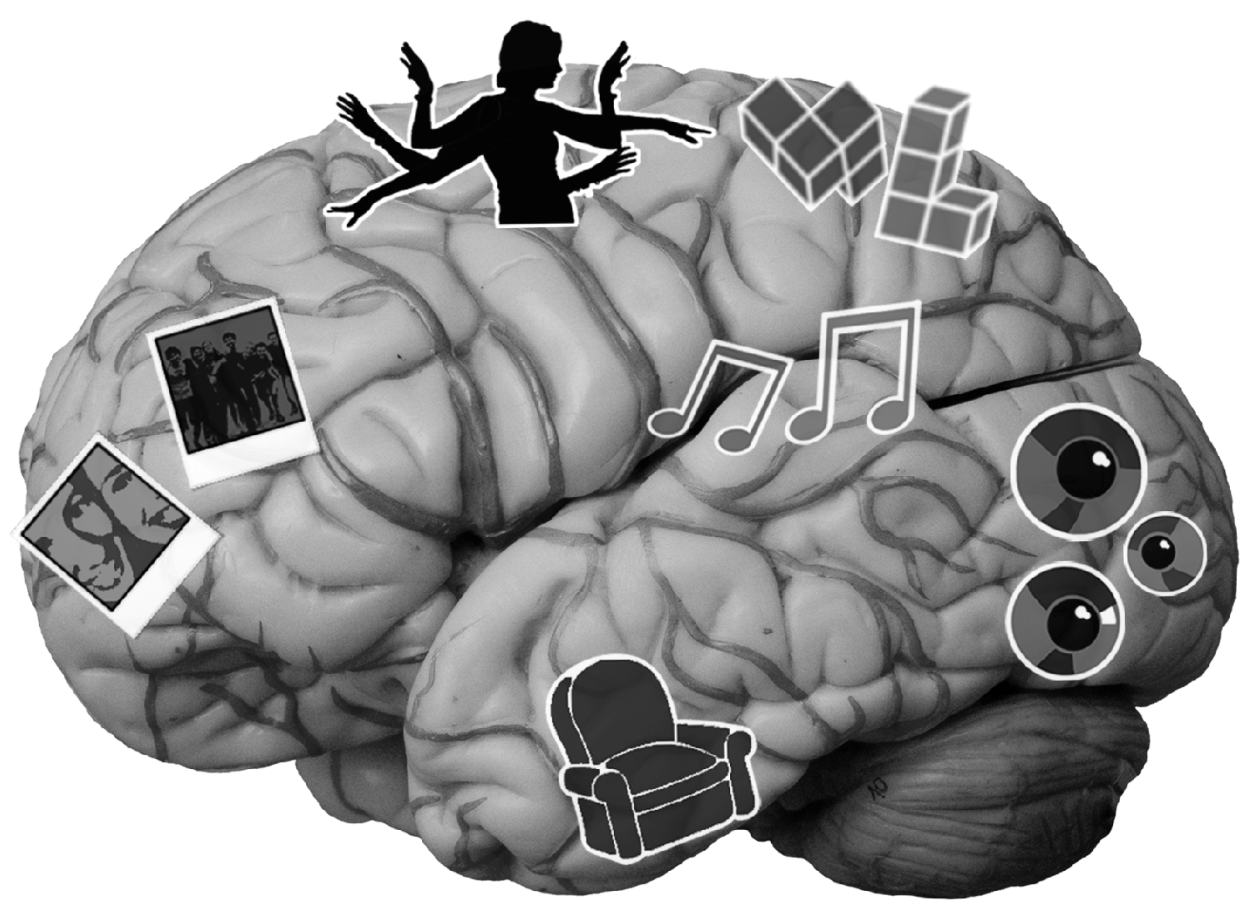

A multi-method investigation into

\author{
the brain dynamics \\ of visual mental imagery
}

Aline de Borst 
Cover design by Aline de Borst

With great help of Marin Been and Kasra Shayesteh Mehr

(C) A.W. de Borst, Maastricht 2011

All rights reserved 


\title{
Imaging imagination
}

\author{
A multi-method investigation into \\ the brain dynamics of visual mental imagery
}

\section{Dissertation}

To obtain the degree of doctor at Maastricht University, on the authority of the Rector Magnificus, prof. dr. G.P.M.F. Mols In accordance with the decision of the Board of Deans,

To be defended in public on Friday the $20^{\text {th }}$ of May 2011 at 12:00 hours

by

Aline Wilhelmina de Borst

Born June 28 1982 in Deventer 


\section{Promotor}

Prof. Dr. F. di Salle

\section{Copromotor}

Dr. E. Formisano

\section{Assessment committee}

Prof. Dr. P.H.M. de Weerd (Chairman)

Dr. V. van Ven

Prof. Dr. D.E.J. Linden (University of Wales, U.K.)

Dr. L. Muckli (University of Glasgow, U.K.) 
The path that you chose has got highs has got lows But it's never what you have in mind Milow - Coming of Age 


\section{Table of contents}

Chapter 1 General introduction

Chapter 2 Mapping the imagery network with time-resolved functional MRI during the mental representation of scenes

Chapter 3 Investigating the spectro-temporal brain dynamics of scene imagery

Chapter 4 Predicting EEG single trials during scene imagery using fMRI and Relevance Vector Machine regression

Chapter 5 Effective connectivity of the mental imagery network 
viii 


\section{Chapter 1}

General introduction 
Visual imagery - the ability to generate and manipulate visual mental representations fulfils a crucial role in everyday human functioning. We employ mental images during various higher cognitive processes, such as creative thinking, remembering events or persons, problem solving or planning (Denis et al., 2001).

Since the early 1970s visual imagery has been a topic of great interest amongst psychologists. Early scientific research focussed on the nature of mental images. Two opposing research groups argued for either a visual, perception-like representation of mental images (Kosslyn, 1980), or an abstract propositional representation (Pylyshyn, 1973). The latter, propositionalists, believe all thinking is propositional in nature and thus every (mental) image can be reduced to such a proposition, which has a non-visual, symbolic or language-like form (Farah, 2000). On the other side, the imagists proposed that there is a visual geometrical code, next to the propositional code. According to the imagists, visual mental representations follow the same laws as real objects in the external world (Kosslyn and Thompson, 2003). These theories gave rise to a lively debate and a stimulation of behavioural and psychophysical research into mental imagery. Most famously, Shepard \& Metzler (1971) showed that reaction times during the mental rotation of 3D objects linearly increased with an increasing rotational angle, suggesting that mental images follow the same laws as real objects.

Nowadays, most studies investigate mental imagery from a neuro-biological perspective, making use of modern neuroimaging techniques. These studies have strengthened the empirical evidence for common neural mechanisms in visual perception and visual mental imagery. In order to understand the similarities and differences between visual mental imagery and visual perception, a short introduction into visual perception will be given, after which the systems involved in mental imagery will be discussed. Furthermore, a short overview of the measurement techniques and methods used in the experiments will be given. Finally, the research questions that gave rise to this dissertation will be discussed.

\subsection{Visual perception}

When light falls through the eye on the retina, the rod and cone receptors transform it into electrical signals. These electrical signals are transferred via the optic nerve and optic chiasm to the Lateral Geniculate Nucleus (LGN), a nucleus of the thalamus, a structure in the centre of the brain. From there the signals travel to the occipital lobe, at the back of the brain. This is called the visual cortex (Goldstein, 1999). The visual cortex is the starting point of visual processing in the cortex. From the visual cortex the signals 
follow two different pathways, the ventral pathway to the temporal lobe and the dorsal pathway to the parietal lobe of the brain (Figure 1.1). The ventral and dorsal pathways each have been attributed with a different function. The ventral pathway is said to be involved in processing of object identity (the "what" pathway) and the dorsal stream is involved in the processing of location and spatial features (the "where" pathway) (Ungerleider and Haxby, 1994). Evidence for this dichotomy comes from decades of research into the monkey and human brain. Tasks involving spatial processing, such as judging the spatial layout of a scene, activated regions in the dorsal pathway. Damage in this part of the brain in monkeys or human patients was found to lead to difficulties in spatial judgements (Mishkin and Ungerleider, 1982; Perenin and Vighetto, 1988). In the ventral pathway many regions have been discovered that respond optimally to certain object categories. For example, seeing faces extensively activates a region in the fusiform gyrus (part of the ventral pathway), while this region responds less optimal to other object categories (Puce et al., 1995). Other regions in the ventral pathway have been identified to be optimally responsive to other object categories such as places, or tools. Whether this suggests a modular organization of ventral temporal cortex, or whether objects are represented in a continuous manner is still under debate (Ishai et al., 1999). In any case, information within these two pathways is not processed in isolation. In fact, a lot of interaction takes place through anatomical connections between the pathways (Umarova et al., 2010).

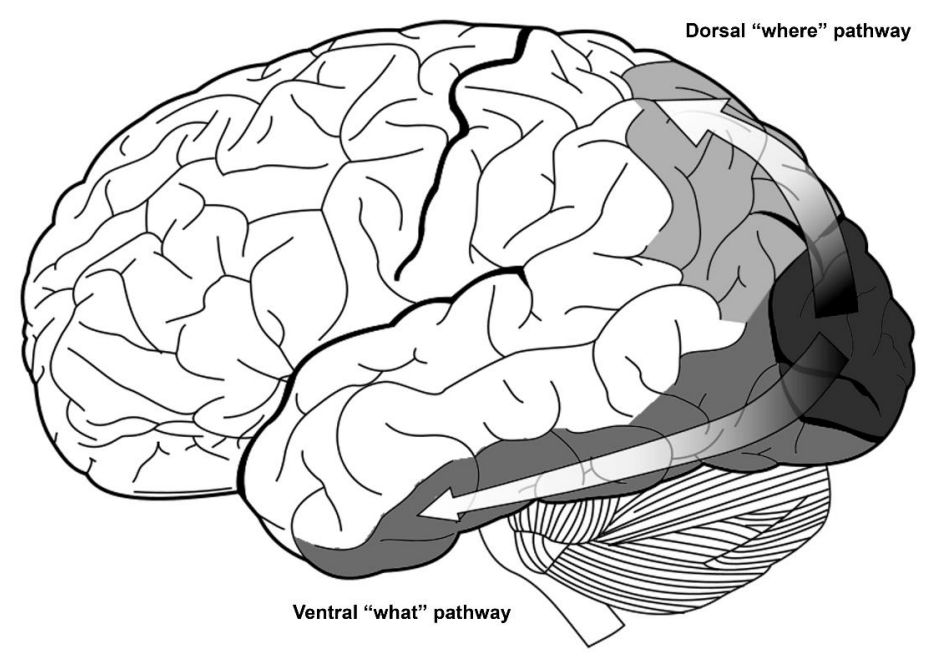

Figure 1.1 Left view of the brain, showing the ventral and dorsal pathway from the occipital lobe. 


\subsection{Visual mental imagery}

A similar organization of object and spatial information into two processing streams has been found during visual mental imagery and in fact, many parallels can be drawn between visual perception and visual imagery. Over the past decades, evidence for common mechanisms in visual perception and visual mental imagery has been provided by psychophysical, neuropsychological and, more recently, neuroimaging research. These studies indicated, e.g., that psychophysically, a visual mental representation can be manipulated in a similar fashion as a visual percept (Shepard and Metzler, 1971) and that patients with imagery deficits often have accompanying deficits in the perceptual domain (Farah, 1984; Trojano and Grossi, 1994). Moreover, neuroimaging studies in healthy individuals showed that the distributed cortical networks active during specific mental imagery tasks are also activated during corresponding perceptual processes (e.g. Kosslyn et al., 1997; Ishai et al., 2000; O'Craven and Kanwisher, 2000; Ganis et al., 2004; Mechelli et al., 2004).

Parts of the organizational structure of mental imagery - such as the division in "what" and "where" processing pathways - are very similar to that of perception, while other aspects, such as the involvement of the early visual cortex, are more disputed. Although mental imagery and perception share certain neural mechanisms, the information flow between brain regions in the visual imagery network differs from visual perception. After all, we do not experience our mental images as being exogenous, but rather as internally generated. To generate, maintain and manipulate these mental images we rely on memory processes in the frontal, parietal and temporal lobes of the brain. Below, the most important components of the visual mental imagery network will be discussed.

\subsection{1 "What" versus "where" in mental imagery}

Analogous to the distinction between the ventral "what" and the dorsal "where" processing pathways as established in visual perception, a dichotomy seems to exist between object imagery and spatial imagery. This dichotomy has been thoroughly investigated using tasks that involve mental processing of object categories or specific visual features and tasks that require imagining spatially coded information.

During object imagery, category-specific regions in the temporal lobe are activated (Ishai et al., 2000; O'Craven and Kanwisher, 2000; Ishai et al., 2002). For instance, Ishai et al. (2000) showed that the mental imagery of faces, houses and chairs 
evoked differential activation in three regions in the ventral temporal cortex, similar to the perception of these categories. O'Craven \& Kanwisher (2000) demonstrated categoryspecific activation for the imagery of houses and faces in, respectively, the parahippocampal gyrus and the fusiform gyrus. These same regions were activated during the perception of places and faces. Compared to perception, the magnitude of Blood Oxygenation Level Dependent (BOLD) responses was smaller during imagery. Ishai et al. (2000) also demonstrated that the volume of activation was more restricted during imagery, compared to perception. Perception and imagery of objects thus activate similar regions in the temporal cortex, but activity is typically more restricted in magnitude and/or volume during imagery.

Like-wise, during tasks that involve imagining spatial information, regions in the dorsal pathway were activated (see a.o. Mellet et al., 1996; Alivisatos and Petrides, 1997; Knauff et al., 2000; Mellet et al., 2000; Formisano et al., 2002; Trojano et al., 2004). Specifically the posterior parietal cortex has shown to play a prominent role in visuospatial imagery. Formisano et al. (2002) showed that comparing the angles of the hands of two imagined clocks evoked bilateral posterior parietal activity. Applying repetitive Transcranial Magnetic Stimulation (TMS) to this same region resulted in a decreased performance on the visual spatial task, hence supporting the important role of the "where" pathway in performing visual spatial imagery (Sack et al., 2002). These studies also found a latency difference between the left and right posterior parietal cortex during spatial imagery, suggesting a separate hemispheric specialization. Confirmed by the results of another TMS study (Sack et al., 2005) it was suggested that the left parietal cortex generated mental images, while the right parietal cortex was involved in the spatial comparison of the mental image.

\subsubsection{Memory and mental imagery}

Memory is an intrinsic part of mental imagery. Memory research distinguishes three important memory processes: encoding, storage and retrieval. Encoding refers to processing of information that needs to be stored. Storage is the outcome of this process, the formation of a long-term representation. Retrieval refers to the re-activation of this representation to form a conscious thought or action. Information can be retrieved from short-term (several seconds to minutes) or long-term (days, years) memory. During mental imagery focus lies on the last of these memory processes, retrieval. After retrieval from memory, images are often retained and manipulated in working memory, depending on task requirements. Working memory encompasses short-term memory, 
according to the influential working memory model of Baddeley (Baddeley and Hitch, 1974; Repovs and Baddeley, 2006).

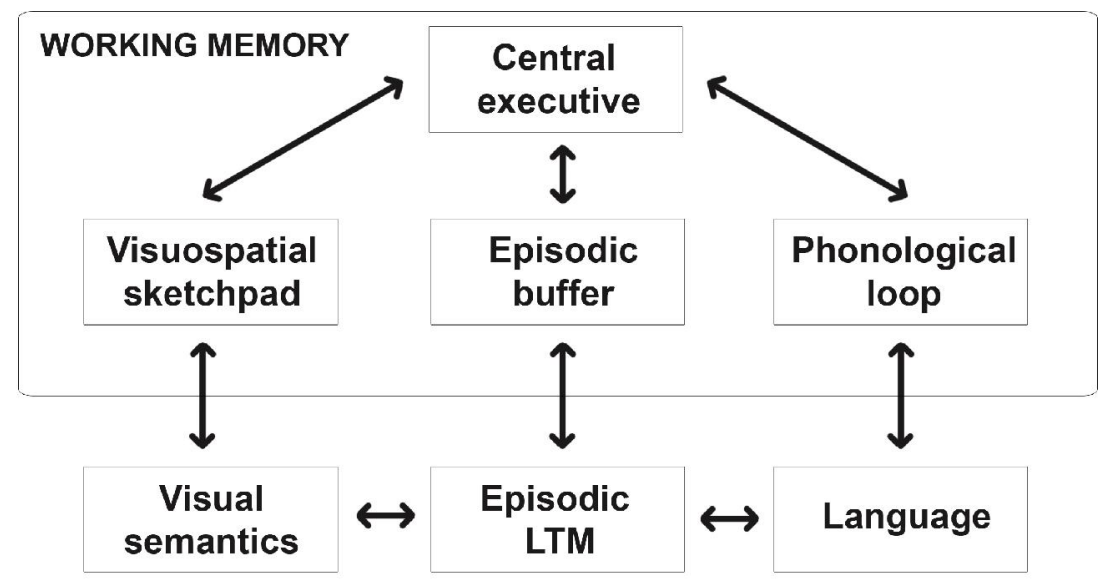

Figure 1.2 The working memory model by Baddeley \& Hitch. Adapted from Repovs \& Baddeley, 2006.

Information enters working memory through perception or retrieval from long term memory. In this multi-component model four elements are distinguished: the central executive, the visuospatial sketchpad, the phonological loop and (in the revised version of the model) the episodic buffer (see Figure 1.2) (Baddeley, 2000; Repovs and Baddeley, 2006). The central executive is proposed to perform a controlling function over the two slave-systems, the visuospatial sketchpad and the phonological loop, and is involved during manipulation of information from these subsystems. The subsystems each provide storage and maintenance for two different memory components: phonological and visuo-spatial information. The dichotomy between these two subsystems has been supported by decades of empirical research (Repovs and Baddeley, 2006). Within the visuo-spatial component another distinction has been made between visual (object) and spatial information. Visual and spatial mental imagery, especially the maintenance of this information, seems to rely on these subcomponents of the working memory model. The newly added component of Baddeley's working memory model, the episodic buffer, forms a link between the two subsystems and long-term memory, by integrating information into a scene or episode (Repovs and Baddeley, 2006).

Although this model provides a strong cognitive basis for experimental research, the neuro-biological underpinnings of the different components are hard to establish. Most likely, no one-to-one link between components and brain regions exists. These components are more likely to arise from a network of interacting brain regions. A meta- 
analysis by Cabeza and Nyberg (2000) reports two major activation sites during working memory: the prefrontal cortex and the parietal cortex (see Figure 1.3). Within the prefrontal cortex, the inferior frontal gyrus (Brodmann Area [BA] 44) seems to be most related to verbal working memory, as part of the phonological loop. Regions in the superior and middle frontal gyrus (BA 9 and 46) are found to be active in working memory tasks involving manipulation and updating of information, a role subscribed to the central executive. Brodmann area 6 is active during all types of working memory tasks, suggesting it to be relevant for non-content specific working memory operations.

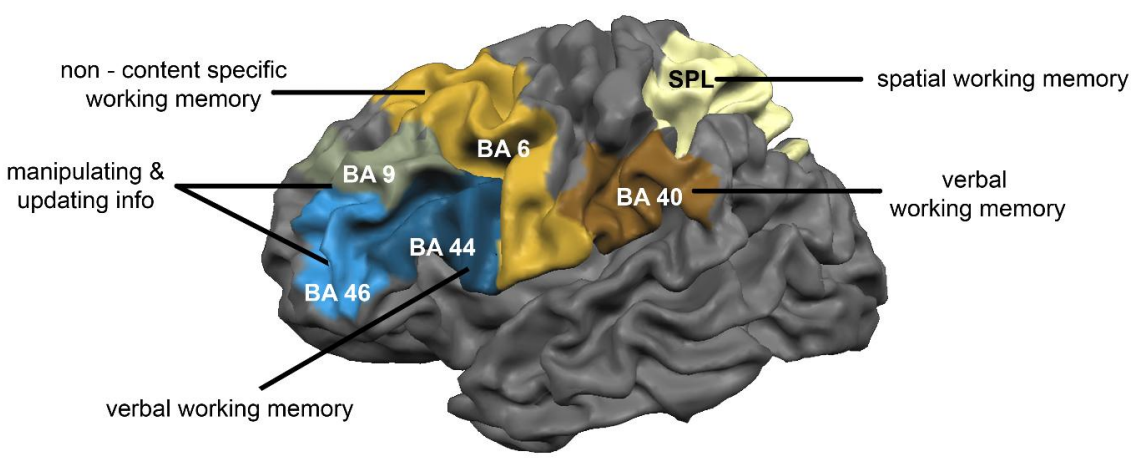

Figure 1.3 Components of the working memory system in the frontal and parietal lobules as identified by Cabeza and Nyberg (2000). BA = Brodmann Area, SPL = superior parietal lobe.

Several studies found that memory retrieval specifically, engages the right prefrontal cortex, while memory encoding involves the left prefrontal cortex (Haxby et al., 1996; Brewer et al., 1998; Cabeza and Nyberg, 2000). During working memory processes, the prefrontal cortex interacts with the parietal cortex.

The parietal cortex is strongly engaged during verbal and spatial working memory tasks. Verbal working memory engages the supramarginal gyrus (BA 40) and is suggested to reflect the phonological store (Awh et al., 1996). Spatial working memory processes, like most spatial tasks, involve the superior parietal cortex. Medially, regions such as the precuneus were engaged during memory retrieval (Shallice et al., 1994; Tulving et al., 1994; Fletcher and Henson, 2001). Several other regions activated during working memory, such as the anterior cingulate cortex and the occipital cortex are likely to reflect non-working memory specific task requirements such as effort and attention.

Electroencephalography (EEG) studies showed additional evidence for the involvement of frontal and parietal sources during working memory and memory retrieval. Left parietal and right frontal ERP effects were found during successful 
memory recollection (Rugg et al., 1998). Left parietal effects were attributed to memory retrieval, while right frontal effects were suggested to arise from the integration of information into an episodic representation (Wilding and Rugg, 1996). This interpretation is in concordance with other EEG studies investigating the spectrotemporal properties of working memory; linking (right) frontal spectral power increases with memory integration and maintenance (Rugg and Venables, 1980; Petsche et al., 1992; Gevins et al., 1997; Jensen and Tesche, 2002).

During visual mental imagery these prefrontal and parietal regions interact with other regions in the parietal cortex and regions in temporal and occipital cortex that are task specific, such as described in the previous and following sections.

\subsubsection{Early visual cortex activation during mental imagery}

The development of modern neuroimaging methods allowed for new ways to contribute to the debate between the propositionalists and the imagists. For example, one could investigate whether early visual cortex is activated during mental imagery. If the early visual cortex is indeed active during mental imagery this would provide strong evidence for a visual image code. The first studies reporting primary visual cortex activation (V1) during a mental imagery task date back to 1993 (Kosslyn et al., 1993; Le Bihan et al., 1993). Since then conflicting results have been found. Several studies found no evidence for activation of the early visual cortex during imagery (e.g. Mellet et al., 1996; Knauff et al., 2000; Mellet et al., 2000; Trojano et al., 2000; Formisano et al., 2002), while others confirmed primary visual cortex activation (e.g. Kosslyn et al., 1999; Thompson et al., 2001; Klein et al., 2004). Kosslyn and Thompson (2003) reviewed many of these studies, containing a variety of different tasks and paradigms, and extracted three essential components underlying early visual cortex activation. According to their analysis three variables explained all systematic differences in the probability of early visual cortex activation across studies: imagining with high resolution details, imagining shapes instead of spatial arrangements and the sensitivity of the measurement technique. Forming a more detailed mental representation or mentally "zooming-in" on a picture might more frequently depend on the early visual cortex, although a study by Thompson et al. (2001) showed no difference in the extent of early visual cortex activation when imagining a lower or higher resolution image. This suggests that the presence of either of these variables by itself might not be enough to find early visual cortex activation, but rather a combination of these variables enhances the probability. Because spatial imagery strongly relies on the parietal cortex and spatial properties are 
topographically stored there (Sereno et al., 2001), this might explain why spatial imagery studies less often report early visual cortex activation. Finally, more sensitive measurements techniques can show more subtle changes in activity. Functional MRI (fMRI) has a better signal to noise ratio, because it needs less averaging of the data across subjects and can easily be replicated over more subjects than positron emission tomography (PET) or single photon emission computed tomography (SPECT). Therefore, fMRI mental imagery studies are more likely to find early visual cortex activation, although the presence or absence of this activation still depends on the specific task. 


\subsection{Measurement techniques}

\subsubsection{Basic principles of functional MRI and EEG}

For the mental imagery experiments in this thesis we used two complementary measurement techniques, functional Magnetic Resonance Imaging (fMRI) and electroencephalography (EEG). Both techniques measure brain activity, but rely on different biological and physical mechanisms. Functional Magnetic Resonance Imaging (fMRI) relies on the principle that neuronal activity causes changes in glucose and oxygen consumption rates, which in turn affect cerebral blood flow, cerebral blood volume and blood oxygenation (Ogawa et al., 1990). These processes influence the measured signal decay process of hydrogen molecules in the blood. The signal decay process of water molecules is measured using radio-frequency (RF) pulses within the static magnetic field of the MRI scanner. In this way active brain regions can be visualized. Because this method relies on blood flow, the measured Blood Oxygenation Level Dependent (BOLD) signal takes several seconds to reach its maximum amplitude and another 8 to 12 seconds to return to baseline. Depending on the acquisition parameters, for example the relaxation time of the hydrogen molecules, the most common temporal resolution of the BOLD signal is 1 to 2 seconds. The spatial resolution also relies i.e. on the choice of acquisition parameters and the magnetic field strength and usually lays around 1 to $4 \mathrm{~mm}^{3}$.

Electroencephalography (EEG) measures the electrical potentials of neurons with electrodes on the skull. The electrical activity as measured on the skull is likely to come from excitatory postsynaptic potentials (EPSP) and inhibitory postsynaptic potentials (IPSP) from pyramidal neurons. When large populations of neurons fire synchronously, the EPSP's give rise to negative waveforms and the IPSP's to positive waveforms (Cooper et al., 1980). Because EEG is measured on the scalp, EEG is most sensitive to neurons closer to the scalp rather than neurons deeper in the brain. Likewise, EEG is most sensitive to neurons that are oriented perpendicular to the scalp. The temporal resolution of EEG is very high, in the millisecond range, depending on the sampling rate during acquisition. The spatial resolution of EEG depends on the ability to spatially segregate the highly correlated and distorted signals as picked up through the skull by the electrodes and the sources they originate from (the inverse problem).

To sum up, fMRI has great precision to spatially localize brain regions involved during a cognitive task, but due to the BOLD lag, has low temporal resolution. Electroencephalography on the other hand, has great temporal precision by its rather direct measurements of neuronal electrical activity, but a more spatially distorted view 
on the neuronal sources underlying that activity (Debener et al., 2006). In two experiments (Chapter 2 and Chapter 3) we exploit the nature of each technique separately to investigate specific characteristics of the mental imagery network.

\subsubsection{Combined EEG-fMRI measurements}

In addition to our separate experiments, we would like to combine the advantages of these two methods and measure with high spatial and temporal resolution simultaneously. Since information from both fields is often still considered separately, integrating spatial and spectro-temporal information of the mental imagery network is challenging territory. One point of consideration is that fMRI and EEG only partially measure the same neuronal sources. As mentioned before, EEG is most sensitive to only a subpopulation of all neurons: those perpendicular and closest to the skull. Furthermore, the EEG signal seems to reflect (a summation of) excitatory and inhibitory postsynaptic potentials that give rise to the firing or inhibition of neurons (Creutzfeldt et al., 1966; Cooper et al., 1980). Functional MRI measures neuronal activity more indirectly, a.o. via blood oxygenation and blood volume in a brain region. The spatial specificity of the signal is thus not directly retraceable to the neurons, as no one to one spatial coupling exists between the neuronal activity and the metabolic changes the BOLD signal arises from (Ugurbil et al., 2003; Harel et al., 2006). What neuronal processes exactly lead to increases in oxygen and glucose metabolism rates and thus an increase in BOLD, is not entirely clear. Studies suggested (Ackermann et al., 1984; Raichle, 1998; Lamm et al., 2001) that both excitatory and inhibitory activity could lead to an increase of BOLD signal, as both influence the regional metabolic energy consumption and blood flow. However, a relationship has also been found between inhibitory activity and a negative BOLD signal (Logothetis, 2008). Besides interpretational issues, there are also some practical problems with measuring simultaneously. One disadvantage of simultaneous stimulation is the degraded EEG signal, due to ballistocardiogram (BCG) and MR-gradient artefacts. These artefacts can be partially corrected for, recovering large segments of the signal.

Keeping in mind the bio-physical underpinnings of these two brain-imaging techniques, one has to be careful interpreting results of (post-hoc) combined EEG-fMRI experiments. However, combining fMRI and EEG measurements within the same experimental paradigm also provides many benefits. Combined EEG-fMRI research helps to study cognitive processes from different angles and ideally offers high spatial and high temporal information of the same process. While this same information could 
be acquired in separate recordings, simultaneous measurements have several other benefits: the sensory stimulation is identical, there is no repetition of information leading to learning effects, and the measurement environment and the "state of the brain", the ongoing fluctuations, are kept constant. These benefits are particularly relevant for mental imagery research, as internally generated content is difficult to control. We use a simultaneous EEG-fMRI approach in Chapter 4 in order to link the spatio-temporal fMRI pattern of Chapter 2 to the spectro-temporal EEG properties of Chapter 3. The analyses of these EEG-fMRI data can be performed in several different manners. Debener et al. (2006) suggested that one way to ensure as much consistency between the two signals as possible is to perform single trial EEG-fMRI analyses, which will be discussed in the next section.

\subsubsection{Single trial EEG-fMRI predictions}

The analyses of combined EEG-fMRI measurements are twofold. On the one hand fMRIconstrained EEG analyses can be performed, in order to improve dipole-estimation of EEG sources (Dale and Halgren, 2001). On the other hand EEG-constrained fMRI analyses can be carried out, to find correlates between fMRI data and EEG power modulations (Ritter and Villringer, 2006). The latter is most powerful in order to investigate dynamic cognitive processes, such as mental imagery. Especially single trial analyses (rather than averaged response analyses) are valuable, because they can capture trial-by-trial fluctuations of brain activity (Debener et al., 2006). By analyzing trial-bytrial coupling between simultaneously measured fMRI and EEG data, an identical attentional state of the participant, repetitions of the task and ongoing brain fluctuations, a.k.a. an identical mental state is ensured.

Most commonly, these single trial EEG-fMRI analyses are performed in a univariate manner. In these univariate analyses predictors (convolved with an hemodynamic response function $[\mathrm{HRF}]$ ) are created from single trial event-related potentials (ERPs) and/or the spectral power in certain frequency bands, which are successively used in a univariate General Linear Model (GLM) of the fMRI data (Debener et al., 2005; Eichele et al., 2005; Scheeringa et al., 2009). This approach however, does not take the multivariate nature of the fMRI data into account. Especially a complex cognitive process as mental imagery (see paragraph 1.2.2) is likely to arise from a distributed pattern of brain activity. Recently, a number of multivariate techniques have been developed to analyze EEG-constrained fMRI data multivariately. One of the latest approaches is the use of predictive modelling in EEG-fMRI data analyses. Predictive 
modelling has been previously applied to fMRI analyses, in which an algorithm is used to learn a functional relationship between the brain response patterns and certain cognitive states (perception of a stimulus, behaviour etc.). After the learning phase, the labelled cognitive states can be predicted from a new dataset (Formisano et al., 2008). Like-wise, this kind of approach can be applied to EEG-fMRI analyses, in which the averaged or single trial ERP or ERSP data can be predicted from a learned functional relationship between these EEG data and the fMRI response patterns (De Martino et al., 2010). This type of multivariate regression can be performed using Relevance Vector Machines (RVM, a machine learning technique). The results of these analyses can give insight in the relationship between multivariate spatial fMRI patterns and modulations in different frequency bands of the EEG. Since specific functions are attributed to different frequency bands and detailed temporal information can be obtained, coupling them to the relevant brain regions will give a deeper insight into the temporal dynamics and underlying functional mechanisms of the mental imagery network. 


\subsection{Research objectives}

The previous paragraphs indicate that mental imagery is a cognitive function that is addressed in many different situations and encompasses a number of sub-systems for e.g. object imagery, spatial imagery and memory, which have been mostly investigated separately. A deeper understanding of our cognitive functioning during mental imagery, however, requires knowledge on how these sub-systems exchange information and work together. This dissertation aims at gathering such knowledge by means of a novel task of natural scene imagery that - besides memory processes - involves aspects of both spatial and object imagery. Furthermore, we use a multimodal approach, gathering knowledge with two complementary measurement techniques (fMRI, EEG).

To optimize comparability and integration of the results we use the same imagery task in a series of fMRI, EEG and simultanous EEG-fMRI studies. In short, the participants learned to associate three different auditory cues with three visual scenes beforehand. During the experiment, participants heard a cue, mentally imagined the corresponding scene and, after a variable delay, judged whether a visually presented fragment of the scene was mirrored or not*.

This task has three major advantages. First, through the use of realistic rather than abstract stimuli, we allowed for more natural, close to life, mental representations. Secondly, with this task we enable activation of object and spatial mental imagery network nodes, as solving the task requires subjects to imagine the objects and their relative spatial layout. This permits us to study these different aspects of imagery simultaneously. Thirdly, without imagining the scene, participants would not be able to make a correct judgement on the target picture. The task therefore ensures the construction and maintenance of the mental image by the participants.

We thouroughly investigated this paradigm with state of the art acquisition and analysis methods that can capture topographical, temporal, relational and predictive properties of this whole-brain network. In each of the chapters we focussed on a different network characteristic, which are briefly discussed below.

*For readers' convenience, the description of the task is repeated in every chapter, mentioning the experiment-specific parameters. 
Topography and BOLD latency information of the scene imagery network

In Chapter 2, we use functional MRI to map the brain regions active during our natural scene imagery task. A BOLD latency mapping procedure is used to look at the temporal organization of the regions. The central result of this study indicates an early onset of frontal regions, with the mesial superior frontal gyrus orchestrating the remote regions in parietal and temporal cortex.

Spectro-temporal properties of the scene imagery network

In Chapter 3, we examine the temporal properties of the scene imagery network and the involved neuronal oscillations using EEG measurements and analyses techniques such as independent component analyses and time-frequency decompositions. Complementing the results of Chapter 2, the EEG data indicate that memory retrieval is supported by theta synchronization at a right frontal site and beta de-synchronization at parietal sites. Theta and beta synchronization of a midline frontal component underlie integration of information into the mental scene.

Predicting EEG single trials during scene imagery from fMRI patterns

In Chapter 4, we use simultaneous EEG-fMRI measurements to investigate the trial-bytrial link between EEG oscillations as reported in chapter 3 and BOLD signal changes as reported in Chapter 2. We use a new technique, Relevance Vector Machine regression, to predict single trial EEG modulations from fMRI patterns. The results reveal that significant predictions of ERPs or ERSPs can be made from fMRI brain patterns. The prediction of centro-frontal ERSP patterns from a network including mesial SFG confirmed the hypothesized coupling as indicated in Chapter 2 and 3. 
Effective connectivity between regions in the scene imagery network

In Chapter 5 we explore how the different nodes in the scene imagery network interact with each other by performing Granger Causality Mapping (GCM), an analysis technique to measure effective connectivity, on fMRI data. We show a specific pattern of information flow through the cortex during our scene imagery task: information flows from the input regions in the auditory cortex, via frontal regions to visual regions in calcarine sulcus, parahippocampal gyrus and parietal cortex.

The chapter includes a neuro-anatomical model of scene imagery that integrates these connectivity findings with previous results of the EEG, fMRI and EEGfMRI studies. This model describes the main nodes of the network, the possible processing flow of information through the network and the patterns of neuronal oscillations that may subserve the exchange of information. It summarizes the knowledge we have gathered with our studies on the functional roles of the regions within the visual mental imagery network and their interconnections. 
Ackermann RF, Finch DM, Babb TL, Engel J, Jr. (1984) Increased glucose metabolism during longduration recurrent inhibition of hippocampal pyramidal cells. J Neurosci 4:251-264.

Alivisatos B, Petrides M (1997) Functional activation of the human brain during mental rotation. Neuropsychologia 35:111-118.

Awh E, Awh E, Jonides J, Smith EE, Schumacher EH, Koeppe RA, Katz S (1996) Dissociation of storage and rehearsal in verbal working memory: Evidence from positron emission tomography. Psychological Science 7:25.

Baddeley A (2000) The episodic buffer: a new component of working memory? Trends Cogn Sci 4:417-423.

Baddeley AD, Hitch G (1974) Working Memory. In: The psychology of learning and motivation: Advances in research and theory (Bower GH, ed), pp 47-89. New York: Academic Press.

Brewer JB, Zhao Z, Desmond JE, Glover GH, Gabrieli JD (1998) Making memories: brain activity that predicts how well visual experience will be remembered. Science 281:1185-1187.

Cabeza R, Nyberg L (2000) Imaging cognition II: An empirical review of 275 PET and fMRI studies. J Cogn Neurosci 12:1-47.

Cooper R, Osselton JW, Shaw JC (1980) EEG technology, 3rd Edition. London: Butterworths.

Creutzfeldt OD, Watanabe S, Lux HD (1966) Relations between EEG phenomena and potentials of single cortical cells. I. Evoked responses after thalamic and erpicortical stimulation. Electroencephalogr Clin Neurophysiol 20:1-18.

Dale AM, Halgren E (2001) Spatiotemporal mapping of brain activity by integration of multiple imaging modalities. Curr Opin Neurobiol 11:202-208.

De Martino F, de Borst AW, Valente G, Goebel R, Formisano E (2010) Predicting EEG single trial responses with simultaneous fMRI and Relevance Vector Machine regression. Neuroimage.

Debener S, Ullsperger M, Siegel M, Engel AK (2006) Single-trial EEG-fMRI reveals the dynamics of cognitive function. Trends Cogn Sci 10:558-563.

Debener S, Ullsperger M, Siegel M, Fiehler K, von Cramon DY, Engel AK (2005) Trial-by-trial coupling of concurrent electroencephalogram and functional magnetic resonance imaging identifies the dynamics of performance monitoring. J Neurosci 25:11730-11737.

Denis M, Logie RH, Cornoldi C, de Vega M, Engelkamp J (2001) Imagery, language and visuospatial thinking. East Sussex: Psychology Press Ltd.

Eichele T, Specht K, Moosmann M, Jongsma ML, Quiroga RQ, Nordby H, Hugdahl K (2005) Assessing the spatiotemporal evolution of neuronal activation with single-trial eventrelated potentials and functional MRI. Proc Natl Acad Sci U S A 102:17798-17803.

Farah MJ (1984) The neurological basis of mental imagery: A componential analysis. Cognition $18: 245$.

Farah MJ (2000) The neural bases of mental imagery. In: The cognitive neurosciences, 2nd Edition (Gazzaniga MS, ed), pp 965-974. Cambridge, MA: MIT Press.

Fletcher PC, Henson RN (2001) Frontal lobes and human memory: insights from functional neuroimaging. Brain 124:849-881. 
Formisano E, De Martino F, Valente G (2008) Multivariate analysis of fMRI time series: classification and regression of brain responses using machine learning. Magn Reson Imaging 26:921934.

Formisano E, Linden DE, Di Salle F, Trojano L, Esposito F, Sack AT, Grossi D, Zanella FE, Goebel R (2002) Tracking the mind's image in the brain I: time-resolved fMRI during visuospatial mental imagery. Neuron 35:185-194.

Ganis G, Thompson WL, Kosslyn SM (2004) Brain areas underlying visual mental imagery and visual perception: An fMRI study. Cognitive Brain Research 20:226.

Gevins A, Smith ME, McEvoy L, Yu D (1997) High-resolution EEG mapping of cortical activation related to working memory: effects of task difficulty, type of processing, and practice. Cerebral Cortex 7:374-385.

Goldstein EB (1999) Sensation \& Perception, 5th Edition. Pacific Grove: Brooks/Cole Publishing Company.

Harel N, Ugurbil K, Uludag K, Yacoub E (2006) Frontiers of brain mapping using MRI. J Magn Reson Imaging 23:945-957.

Haxby JV, Ungerleider LG, Horwitz B, Maisog JM, Rapoport SI, Grady CL (1996) Face encoding and recognition in the human brain. Proc Natl Acad Sci U S A 93:922-927.

Ishai A, Ungerleider LG, Haxby JV (2000) Distributed neural systems for the generation of visual images. Neuron 28:979-990.

Ishai A, Haxby JV, Ungerleider LG (2002) Visual imagery of famous faces: effects of memory and attention revealed by fMRI. Neuroimage 17:1729-1741.

Ishai A, Ungerleider LG, Martin A, Schouten JL, Haxby JV (1999) Distributed representation of objects in the human ventral visual pathway. Proc Natl Acad Sci U S A 96:9379-9384.

Jensen O, Tesche CD (2002) Frontal theta activity in humans increases with memory load in a working memory task. European Journal of Neuroscience 15:1395-1399.

Klein I, Dubois J, Mangin JF, Kherif F, Flandin G, Poline JB, Denis M, Kosslyn SM, Le Bihan D (2004) Retinotopic organization of visual mental images as revealed by functional magnetic resonance imaging. Brain Res Cogn Brain Res 22:26-31.

Knauff M, Kassubek J, Mulack T, Greenlee MW (2000) Cortical activation evoked by visual mental imagery as measured by fMRI. Neuroreport 11:3957-3962.

Kosslyn SM (1980) Image and mind. Cambridge, MA: Harvard University Press.

Kosslyn SM, Thompson WL (2003) When is early visual cortex activated during visual mental imagery? Psychol Bull 129:723-746.

Kosslyn SM, Thompson WL, Alpert NM (1997) Neural systems shared by visual imagery and visual perception: a positron emission tomography study. Neuroimage 6:320-334.

Kosslyn SM, Kosslyn SM, Alpert NM, Thompson WL, Maljkovic V (1993) Visual mental imagery activates topographically organized visual cortex: PET investigations. Journal of Cognitive Neuroscience 5:263.

Kosslyn SM, Pascual-Leone A, Felician O, Camposano S, Keenan JP, Thompson WL, Ganis G, Sukel KE, Alpert NM (1999) The role of area 17 in visual imagery: convergent evidence from PET and rTMS. Science 284:167-170. 
Lamm C, Windischberger C, Leodolter U, Moser E, Bauer H (2001) Evidence for premotor cortex activity during dynamic visuospatial imagery from single-trial functional magnetic resonance imaging and event-related slow cortical potentials. Neuroimage 14:268-283.

Le Bihan D, Turner R, Zeffiro TA, Cuenod CA, Jezzard P, Bonnerot V (1993) Activation of human primary visual cortex during visual recall: a magnetic resonance imaging study. Proc Natl Acad Sci U S A 90:11802-11805.

Logothetis NK (2008) What we can do and what we cannot do with fMRI. Nature 453:869-878.

Mechelli A, Price CJ, Friston KJ, Ishai A (2004) Where bottom-up meets top-down: neuronal interactions during perception and imagery. Cerebral Cortex 14:1256-1265.

Mellet E, Tzourio N, Crivello F, Joliot M, Denis M, Mazoyer B (1996) Functional anatomy of spatial mental imagery generated from verbal instructions. Journal of Neuroscience 16:6504-6512.

Mellet E, Briscogne S, Tzourio-Mazoyer N, Ghaem O, Petit L, Zago L, Etard O, Berthoz A, Mazoyer B, Denis M (2000) Neural correlates of topographic mental exploration: the impact of route versus survey perspective learning. Neuroimage 12:588-600.

Mishkin M, Ungerleider LG (1982) Contribution of striate inputs to the visuospatial functions of parieto-preoccipital cortex in monkeys. Behav Brain Res 6:57-77.

O'Craven KM, Kanwisher N (2000) Mental imagery of faces and places activates corresponding stiimulus-specific brain regions. Journal of Cognitive Neuroscience 12:1013-1023.

Ogawa S, Lee TM, Kay AR, Tank DW (1990) Brain magnetic resonance imaging with contrast dependent on blood oxygenation. Proc Natl Acad Sci U S A 87:9868-9872.

Perenin MT, Vighetto A (1988) Optic ataxia: a specific disruption in visuomotor mechanisms. I. Different aspects of the deficit in reaching for objects. Brain 111 ( Pt 3):643-674.

Petsche H, Lacroix D, Lindner K, Rappelsberger P, Schmidt-Henrich E (1992) Thinking with images or thinking with language: a pilot EEG probability mapping study. International Journal of Psychophysiology 12:31-39.

Puce A, Allison T, Gore JC, McCarthy G (1995) Face-sensitive regions in human extrastriate cortex studied by functional MRI. J Neurophysiol 74:1192-1199.

Pylyshyn ZW (1973) What the mind's eye tells the mind's brain: A critique of mental imagery. Psychological Bulletin 80:1.

Raichle ME (1998) Behind the scenes of functional brain imaging: a historical and physiological perspective. Proc Natl Acad Sci U S A 95:765-772.

Repovs G, Baddeley A (2006) The multi-component model of working memory: explorations in experimental cognitive psychology. Neuroscience 139:5-21.

Ritter P, Villringer A (2006) Simultaneous EEG-fMRI. Neurosci Biobehav Rev 30:823-838.

Rugg MD, Venables PH (1980) EEG correlates of the acquisition of high- and low-imagery words. Neuroscience Letters 16:67-70.

Rugg MD, Mark RE, Walla P, Schloerscheidt AM, Birch CS, Allan K (1998) Dissociation of the neural correlates of implicit and explicit memory. Nature 392:595-598.

Sack AT, Camprodon JA, Pascual-Leone A, Goebel R (2005) The dynamics of interhemispheric compensatory processes in mental imagery. Science 308:702-704. 
Sack AT, Sperling JM, Prvulovic D, Formisano E, Goebel R, Di Salle F, Dierks T, Linden DE (2002) Tracking the mind's image in the brain II: transcranial magnetic stimulation reveals parietal asymmetry in visuospatial imagery. Neuron 35:195-204.

Scheeringa R, Petersson KM, Oostenveld R, Norris DG, Hagoort P, Bastiaansen MC (2009) Trial-bytrial coupling between EEG and BOLD identifies networks related to alpha and theta EEG power increases during working memory maintenance. Neuroimage 44:1224-1238.

Sereno MI, Pitzalis S, Martinez A (2001) Mapping of contralateral space in retinotopic coordinates by a parietal cortical area in humans. Science 294:1350-1354.

Shallice T, Shallice T, Fletcher P, Frith CD, Grasby P (1994) Brain regions associated with acquisition and retrieval of verbal episodic memory. Nature 368:633.

Shepard RN, Metzler J (1971) Mental rotation of three-dimensional objects. Science 171:701.

Thompson WL, Kosslyn SM, Sukel KE, Alpert NM (2001) Mental imagery of high- and lowresolution gratings activates area 17 . Neuroimage 14:454-464.

Trojano L, Grossi D (1994) A critical review of mental imagery defects. Brain and Cognition 24:213.

Trojano L, Trojano L, Linden DEJ, Formisano E, Goebel R, Sack AT, Di Salle F (2004) What clocks tell us about the neural correlates of spatial imagery. European Journal of Cognitive Psychology 16:653.

Trojano L, Grossi D, Linden DE, Formisano E, Hacker H, Zanella FE, Goebel R, Di Salle F (2000) Matching two imagined clocks: the functional anatomy of spatial analysis in the absence of visual stimulation. Cerebral Cortex 10:473-481.

Tulving E, Kapur S, Markowitsch HJ, Craik FI, Habib R, Houle S (1994) Neuroanatomical correlates of retrieval in episodic memory: auditory sentence recognition. Proc Natl Acad Sci U S A 91:2012-2015.

Ugurbil K, Toth L, Kim DS (2003) How accurate is magnetic resonance imaging of brain function? Trends Neurosci 26:108-114.

Umarova RM, Saur D, Schnell S, Kaller CP, Vry MS, Glauche V, Rijntjes M, Hennig J, Kiselev V, Weiller C (2010) Structural connectivity for visuospatial attention: significance of ventral pathways. Cereb Cortex 20:121-129.

Ungerleider LG, Haxby JV (1994) 'What' and 'where' in the human brain. Curr Opin Neurobiol 4:157-165.

Wilding EL, Rugg MD (1996) An event-related potential study of recognition memory with and without retrieval of source. Brain 119 ( Pt 3):889-905. 


\section{Chapter 2}

\section{Mapping the imagery network with time-resolved functional MRI during the mental representation of scenes}

\section{Abstract}

Recalling to mind a visual scene is a common mental operation. It requires both the mental representation of the objects that form the scene and their spatial arrangement. Here we unravel the topography and dynamics of brain activation during a behaviourally-controlled paradigm that requires the imagery of complex visual scenes. With functional MRI (fMRI) we localized the functional network of activated brain regions that includes the mesial superior frontal gyrus (SFG), the right middle frontal gyrus (MFG), the calcarine sulcus, bilateral parietal regions and the parahippocampal gyrus. The BOLD latency information reveals an earlier onset of frontal regions and subcortical structures compared to ventro-temporal and parietal regions. These early frontal regions work closely together though with the remote parietal regions to encode the spatial sketch of the scene and with occipital-temporal regions to encode the detailed visual representations of the objects. The relation between subjects' behavioural performances and brain activations during mental imagery demonstrate the crucial role of mesial SFG in forming and maintaining the mental image of the scene.

\section{Based on}

A.W. de Borst, A.T. Sack, B.M. Jansma, F. Esposito, F. de Martino, G. Valente, A. Roebroeck, F. di Salle, R. Goebel, and E. Formisano. Integration of "what" and "where in frontal cortex during visual imagery of scenes. (submitted). 


\subsection{Introduction}

Visual mental imagery fulfils a crucial role in everyday human functioning. During various higher cognitive processes, such as creative thinking, problem solving and planning, we employ mental representations (Denis et al., 2001). The ability to generate and manipulate visual mental representations is called visual mental imagery. Originally, visual mental imagery was investigated with behavioural methods, in both healthy subjects and patients with brain lesions (Shepard and Metzler, 1971; Kosslyn et al., 1983; Farah, 1984; Finke, 1985). Patients with visual deficits, such as prosopagnosia (e.g. inability to recognize faces) displayed similar dysfunctions in the imagery domain (Barton and Cherkasova, 2003). This indicated that visual mental imagery and perception might share common neural processes (Farah, 1984; Trojano and Grossi, 1994). This idea was further confirmed by psychophysical research. For example, Shepard and Metzler (1971) showed that reaction times during the mental rotation of 3D objects linearly increased with an increasing rotational angle, suggesting that a visual mental representation can be manipulated in a similar fashion as a visual percept (Shepard and Metzler, 1971).

With the advent of modern neuroimaging techniques, the neural substrates of visual mental imagery could be investigated non-invasively in healthy subjects. Indeed, neuroimaging studies on mental imagery over the last decades have shown that our capability to generate, evaluate and manipulate mental representations relies on certain distributed cortical networks that are also activated during corresponding perceptual processes (e.g. Kosslyn et al., 1997; Ishai et al., 2000; O'Craven and Kanwisher, 2000; Ganis et al., 2004; Mechelli et al., 2004).

A distinction has been delineated between neural mechanisms underlying tasks that involve mental processing of object categories or specific visual features and tasks that require imagining spatially coded information. Analogous to the distinction between the ventral "what" and the dorsal "where" processing streams in visual perception, a dichotomy seems to exist between object imagery and spatial imagery. In fact, several studies observed activity in the temporal category-specific areas during visual object imagery (Ishai et al., 2000; O'Craven and Kanwisher, 2000; Ishai et al., 2002). For instance, the mental imagery of houses, faces, and chairs evoked activity in segregated regions in ventro-temporal cortex. These regions are also active during the visual perception of these same categories (Ishai et al., 2000). A difference between perception and imagery in these regions can be observed in the magnitude and level of activation. Generally, the amplitude of blood oxygen level dependent (BOLD) responses is lower and the magnitude of activation is smaller during imagery than during 
perception of comparable stimuli (Ishai et al., 2000; O'Craven and Kanwisher, 2000).

In a similar vein, during visual spatial imagery many neuroimaging studies found activity along the dorsal pathway (e.g. Mellet et al., 1996; Alivisatos and Petrides, 1997; Knauff et al., 2000; Mellet et al., 2000b; Formisano et al., 2002; Trojano et al., 2004). This suggests that the fronto-parietal network, activated during perceptual visuospatial tasks, also underlies the spatial analysis of mentally imagined objects or scenes. For instance, the posterior parietal lobe, which performs an important functional role in spatial perception, has been found to fulfil a central function in spatial processing of imagined material as well (Alivisatos and Petrides, 1997; Goebel et al., 1998; Mellet et al., 2000b; Trojano et al., 2000; Formisano et al., 2002; Podzebenko et al., 2002; Sack et al., 2002; Trojano et al., 2002). More specifically, the left and right posterior parietal cortices (PPC) seem to have distinctive roles during visual mental imagery. Functional Magnetic Resonance Imaging (fMRI) and Transcranial Magnetic Stimulation (TMS) studies by Formisano et al. (2002) and Sack et al. (2002) revealed a latency difference between left (early) and right (late) PPC during a mental clock task. They furthermore showed impaired performance after repetitive TMS in right PPC during the same task, suggesting different functional roles for left and right PPC. The converging evidence seems to suggest that during spatial image construction bilateral PPC is involved, while image inspection is mostly confined to the right PPC.

Although visual perception of objects and spatial configurations and visual imagery of these same representations show extensive overlap in activation patterns, activation of early visual cortex during visual mental imagery has been a matter of lively debate. The first reports of early visual cortex activation during tasks requiring the recollection or imagery of a visual stimulus date back to 1993 (Kosslyn et al., 1983; Le Bihan et al., 1993). Since then studies found contradictory results, either reporting activation of early visual cortex (e.g. Kosslyn et al., 1999; Thompson et al., 2001; Klein et al., 2004) or the absence of any activity in primary visual cortex during visual mental imagery (e.g. Mellet et al., 1996; Knauff et al., 2000; Mellet et al., 2000b; Trojano et al., 2000; Formisano et al., 2002). A meta-analysis of Kosslyn and Thompson (2003) suggested that a combination of three variables, imagining with high-resolution details, imagining shapes instead of spatial arrangements and the sensitivity of the measurement technique leads to an increased chance of activating primary visual cortex during visual imagery.

So far, visual object and visual spatial imagery have mostly been investigated separately. Studies have been able to locate regions that are activated specifically during object-oriented or spatially defined tasks. In the current study we want to combine the imagery of objects with the imagery of spatially coded information in order to 
investigate the properties of the common imagery network of brain regions. In order to do so, we have designed a behaviourally controlled task of realistic scene imagery during which participants are required to imagine detailed objects are well as their spatial arrangements. We hypothesize to find activated regions in ventro-temporal cortex as well as parietal cortex. Furthermore, we are interested to explore whether early visual cortex will be activated by a task of combined object and spatial imagery, requiring the detailed analysis of scenes. Finally, we want to elaborate on the current literature by investigating the functional properties of this common scene imagery network by exploring the latency information of the network nodes. 


\subsection{Methods}

\subsubsection{Participants}

Ten healthy right- handed volunteers (8 female, mean age 22.2 years; range 18-26) participated in the fMRI study. All participants had normal or corrected-to-normal vision. The local ethical committee approved the study and participants gave their written informed consent before participation.

\subsubsection{Task and stimuli}

Twenty-seven digital colour photographs were used as stimuli (screen and picture resolution 1024 by 768 pixels). Three photographs (see example in Figure 2.1) depicted the interior of a room and comprised several pieces of furniture. These photographs defined the visual imagery content and were solely used during a training phase. Twelve photographs (targets) were fragments of the three original scenes (four per scene), containing a minimum of two partly visible objects. The other twelve photographs (targets) were these same images mirrored across the horizontal axis (see Figure 2.1). The default image was a grey background ( $50 \%$ black, $50 \%$ white). All photographs contained a centralized white fixation cross and were presented at a 24 by $30^{\circ}$ visual angle.

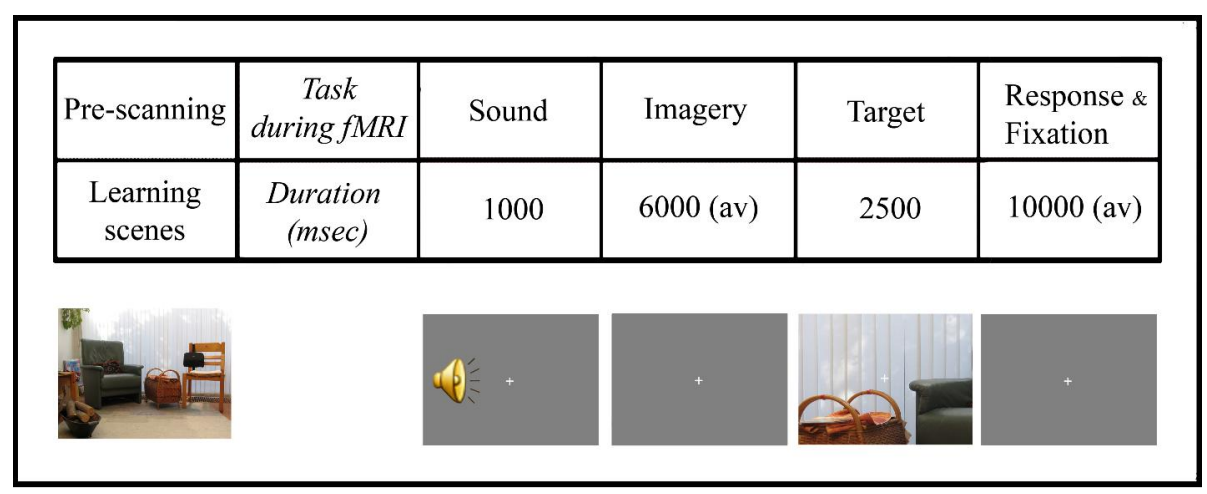

Figure 2.1 Stimulus protocol. Schematic overview of the temporal course of one trial in milliseconds $(\mathrm{ms})$. (av) = on average.

The fMRI experiment consisted of 54 (3 associations x 18 repetitions) trials. As depicted in Figure 2.1, each trial of the experiment started with an auditory cue. The three 
auditory stimuli were one second (s) sound clips of a female speaker uttering "photo one", "photo two" or "photo three". During the subsequent interval participants imagined the scene that they previously had learned to associate with the cue. After a variable delay $(5,6$, or $7 \mathrm{~s}$, average $=6 \mathrm{~s})$ a mirrored or non-mirrored fragment of the scene (50-50\% distribution) was visually presented as a target for $2.5 \mathrm{~s}$. After each target participants pressed a button with their right middle or index finger to indicate whether the fragment matched the imagined scene, or was mirrored. All trials ended with a fixation period ( 8 - $12 \mathrm{~s}$ ) during which the participants performed no task. The interval lengths were pseudo-randomized over trials and the occurrence of each period length was equally distributed over the three types of trials (scene one, two and three) and type of target picture. The trials (e.g. the order of the tones and target pictures) were pseudorandomized.

Prior to fMRI measurements, participants were trained on imagining three scenes and associating these scenes with the three auditory cues. The training session consisted of 24 randomized trials of simultaneous presentation of the auditory stimulus and the corresponding visual scene (5s), followed by a fixation period (6s) during which the participants imagined the scene. After training, participants received task instructions and were asked to vividly visualize the correct scene after each cue in the main experiment, while fixating at the fixation cross.

\subsubsection{Data acquisition}

A 3T Siemens MR (head) scanner (MAGNETOM Allegra, Siemens Medical Systems, Erlangen, Germany) was used for imaging. Functional scans were acquired with a Gradient Echo Echo Planar Imaging sequence with a Repetition Time (TR) of 1500 ms and an Echo Time (TE) of $30 \mathrm{~ms}$. For each of the two functional runs 370 volumes were acquired composed of 23 slices (matrix $=64 \times 64$, voxel dimensions $=3 \times 3 \times 4.5 \mathrm{~mm} 3$, interslice time $=65 \mathrm{~ms}$, flip angle $=90^{\circ}$ ). Between the two functional runs high resolution T1- weighted structural images of the whole brain were acquired ( 3 subjects: MPRAGE 192 slices, matrix $=256 \times 256$, voxel dimensions $=1 \times 1 \times 1 \mathrm{~mm} 3, \mathrm{TR}=2300 \mathrm{~ms}, \mathrm{TE}=3.93$ ms, flip angle $=12^{\circ} ; 7$ subjects: MDEFT 176 slices, matrix $=256 \times 256$, voxel dimensions $=$ $1 \times 1 \times 1 \mathrm{~mm} 3, \mathrm{TR}=7.92 \mathrm{~ms}, \mathrm{TE}=2.40 \mathrm{~ms}$, flip angle $=15^{\circ}$ ). 


\subsubsection{Data analyses}

Preprocessing

All functional MRI data were analysed using fMRI analysis and visualisation software BrainVoyager QX (Brain Innovation B.V., Maastricht, The Netherlands). Functional data were 3D motion corrected (trilinear interpolation), corrected for slice scan time differences and temporally filtered (high pass: 5 cycles/run cut-off). No spatial filtering was applied. The anatomical data were corrected for intensity inhomogeneity (Goebel et al., 2006) and transformed into Talairach space (Talairach and Tournoux, 1988). The functional data were then aligned with the anatomical data and transformed into the same space, to create $4 \mathrm{D}$ volume time-courses (VTCs). The anatomical data were used for surface reconstruction of both hemispheres in all subjects (description of cortex segmentation in Goebel et al., 2006).

\section{Latency mapping}

Statistical modelling of the time-courses was based on a general linear model with a design matrix implementing a deconvolution analysis. An estimate of the hemodynamic response at each TR $(\mathrm{TR}=1.5 \mathrm{~s})$ during a $19.5 \mathrm{~s}$ window after trial onset was obtained using a separate stick predictor. The group map depicting the spatio-temporal pattern of BOLD activation (Figure 2.2) was based on the concatenation of single-subject deconvolution matrices (fixed effect GLM). In this map, a location was considered to be active during mental imagery if the significance of the statistical contrast including the first five stick predictors (preceding the onset of the target) was below the voxel-wise threshold of $\mathrm{p}$ (uncorrected) $<0.0001$. Maps of BOLD response latency were obtained by colour coding significantly active voxels according to the earliest stick predictor for which the beta weights reached significance (i.e. a dark green colour indicates a significant beta weight for the stick predictor corresponding to the first TR after sound onset, a lighter green to second TR, etc.). Regions of interest (ROIS) were selected based on the group map and presence in individual subject maps.

In order to quantify the BOLD latency with sub-TR millisecond resolution, a nonlinear analytical procedure (BOLD Latency Mapping) was applied to BOLD response data in the ROIs (see Figure 2.3B). For each ROI, a time- course was obtained by spatially averaging the voxel time-courses and then temporally interpolating it by a factor of 3 . Then, a piece-wise linear (PWL) fitting (pseudo-trapezoid) procedure with four break- 
points was applied to the resulting time-courses. The estimates of the first break point were used for the onset time of each ROI response (Richter et al., 2000; Formisano et al., 2002). In the PWL fitting procedure the initial and final baseline levels were not constrained to be identical since the epochs were cut off before target onset (i.e. no return to initial baseline was forced in the PWL fit before the end of the time window of interest).

\section{Retinotopic mapping}

Retinotopic data (polar angle mapping) of one subject were acquired in a previous session (Been et al., in preparation). These functional data were aligned with the withinsession anatomical data. Then, the anatomical data from the previous session were aligned with the ACPC transformed anatomical data of the current study. Using the coregistration files of the functional-anatomical intra-session alignment (retinotopy data), the ACPC translation/rotation file between anatomical sessions, and the Talairach transformation file of the current session, the functional retinotopy data were aligned with the anatomical data of the current study and transformed into $4 \mathrm{D}$ volume timecourses. A cross correlation map with 14 lags was calculated in order to visualize the borders of the visual areas (for more information see Sereno et al., 1995; Warnking et al., 2002). These data were overlaid on a flattened representation of the cortex, cut along the calcarine sulcus in order to represent the data optimally. Finally, the mental imagery data of this subject were overlaid on the retinotopic map (see Figure 2.4).

\section{Reaction time based fMRI analyses}

For each participant, reaction times (onset time button-press minus onset time target) of the correct trials were calculated. Trials were ordered according to response latencies and a median split was applied, resulting in a group of "fast" and "slow" trials per participant. Functional MRI trials were labelled based on these two categories and compared. An estimate of the hemodynamic response at each imagery and target picture interval for the fast and slow conditions was obtained using a boxcar predictor convolved with a hemodynamic response function. A fixed effect GLM was performed with a contrast testing for a difference in BOLD signal during the imagery periods followed by fast or slow responses, thresholded using a False Discovery Rate (FDR) (Genovese et al., 2002) of 0.05 (see Figure 2.5). 


\subsection{Results}

All fMRI results are solely based on the imagery period during the trials, excluding target picture information.

\subsubsection{Behavioural results}

Participants $(\mathrm{N}=10)$ responded correctly on 92 percent $(\mathrm{SD}=10.2)$ of the non- mirrored trials and 88 percent $(\mathrm{SD}=11.3)$ of the mirrored trials with an average reaction time of $1823 \mathrm{~ms}(\mathrm{SD}=642.3)$ for non-mirrored and $2008 \mathrm{~ms}(\mathrm{SD}=808.6)$ for mirrored trials. A paired sampled $t$-test showed that responses were significantly faster $(t(9)=-2.32, p<$ $0.046)$ and more accurate $(\mathrm{t}(9)=2.34, \mathrm{p}<0.044)$ during the non- mirrored trials.

\subsubsection{Latency mapping}

Latency mapping of fMRI data (Figure 2.2 and Figure 2.3B) showed that the auditory cortex (AC) and superior temporal gyrus (STG) were activated earliest, together with parts of the thalamus (Th), putamen $(\mathrm{Pu})$, the mesial superior frontal gyrus (SFG) (extending from the supplementary motor area to the anterior cingulate cortex) and premotor (PreM) areas (dark green). Then, more extensive clusters in the SFG, pre-motor cortex, thalamus and putamen became active as well as the right medial frontal gyrus (MFG), insula (INS), the calcarine sulcus (CalS) and parts of the (mostly left) superior parietal lobe (SPL), precuneus, intraparietal sulcus and parieto-occipital sulcus (light green to yellow). Subsequently, large parts of the (right) SPL, precuneus, medial and inferior occipital cortex and the parahippocampal gyrus (PHG) became active (orange). Last, parts of the right SPL, including intraparietal sulcus, and part of the right PHG were activated (dark orange to red). For an overview of all significantly activated regions see Table 2.1.

\subsubsection{Hemodynamic responses}

The ROI time-courses (Figure 2.3A) visualize the different shapes and onsets of the hemodynamic responses in the active regions. The BOLD signal from the auditory cortex (AC) showed a steep increase with the onset of the auditory cue at the first TR. After the 


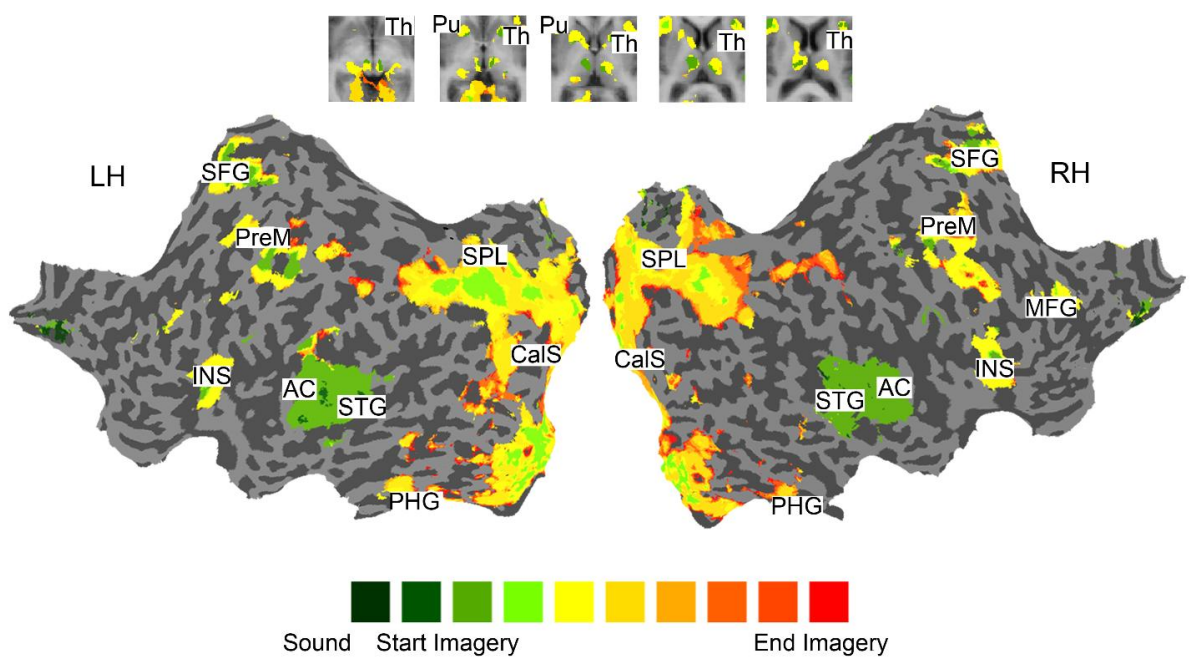

Figure 2.2 Multisubject (deconvolution) map during the mental imagery of scenes superimposed on a flattened representation of the cortical sheet. The voxels are colour coded according to their first significant beta value, representing the latency of BOLD activation during the auditory cue and mental imagery part of the trial. Top: Th = thalamus, $\mathrm{Pu}=$ putamen . Left to right: $\mathrm{INS}=$ insula, $\mathrm{SFG}=$ mesial superior frontal gyrus, PreM = premotor cortex, $\mathrm{AC}=$ auditory cortex, $\mathrm{STG}=$ superior temporal gyrus, $\mathrm{PHG}=$ parahippocampal gyrus, $\mathrm{SPL}=$ superior parietal lobule, CalS $=$ calcarine sulcus,$M F G=$ medial frontal gyrus .

signal peaked around the third TR it showed a sharp decrease. The BOLD signal of the superior temporal gyrus (STG) followed quickly after the auditory cortex response, displaying a similar shape. With the onset of the imagery period, various areas showed an increase in BOLD signal around the second TR.
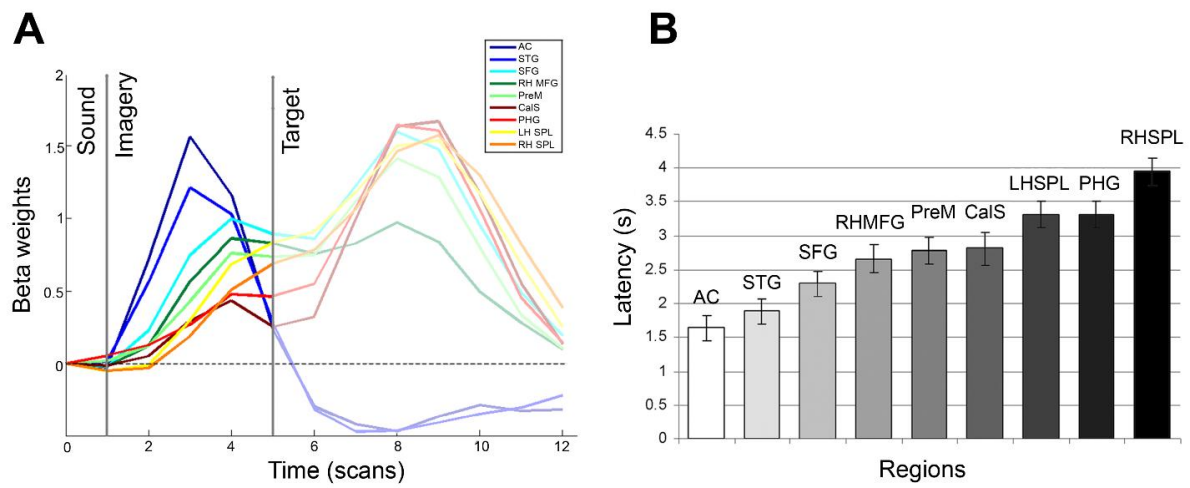

Figure 2.3 Functional MRI latency information. A. Event-related average time-courses of nine regions of interest during mental imagery (in volumes). B. Estimated latency in seconds of nine regions of interest. Abbreviations as in Figure 2.2. 
The mesial superior frontal gyrus (SFG) and the right medial frontal gyrus (RH MFG) showed the steepest increase, closely followed by the premotor cortex (PreM). Last, the parahippocampal gyrus (paraHG), the calcarine sulcus (CalS) and the left and right superior parietal lobe (SPL) showed an increase in BOLD signal. Due to similar BOLD shape, a distinguishable latency difference between left and right SPL could be observed. The onset estimates provided by the BOLD Latency Mapping (BLM) analysis of the BOLD responses confirmed the described latency pattern of activation (Figure 2.3B).

With the sole exception of the auditory regions, all regions were involved during both imagery and target processing (data not shown) as reflected by the regional BOLD signal. Similar to studies that compared imagery and perception conditions (O'Craven and Kanwisher, 2000; Ganis et al., 2004), the BOLD signal change was lower in amplitude during the imagery condition than during the perception of the target.

\subsubsection{Calcarine sulcus activity}

In 8 out of 10 subjects activity along the calcarine sulcus was observed during the mental imagery of scenes. In Figure 2.4 the calcarine sulcus activity of a single participant is presented. The brain activity during imagery (target-related activity is excluded) is overlaid in purple on a retinotopic map of the participant (acquired in an earlier session), showing V1 involvement. Time courses corresponding to patches in left and right V1 are shown on the left and right side of Figure 2.4, reflecting the BOLD signal change during imagery and after the visual target (target onset indicated by red line). The time courses show a clear increase in BOLD signal during the imagery period, with a small decrease at the end of the period, followed by a strong increase with the onset of the target. The lower maps in Figure 2.4 show an extensive imagery- related activation of V1 and higher visual areas. 


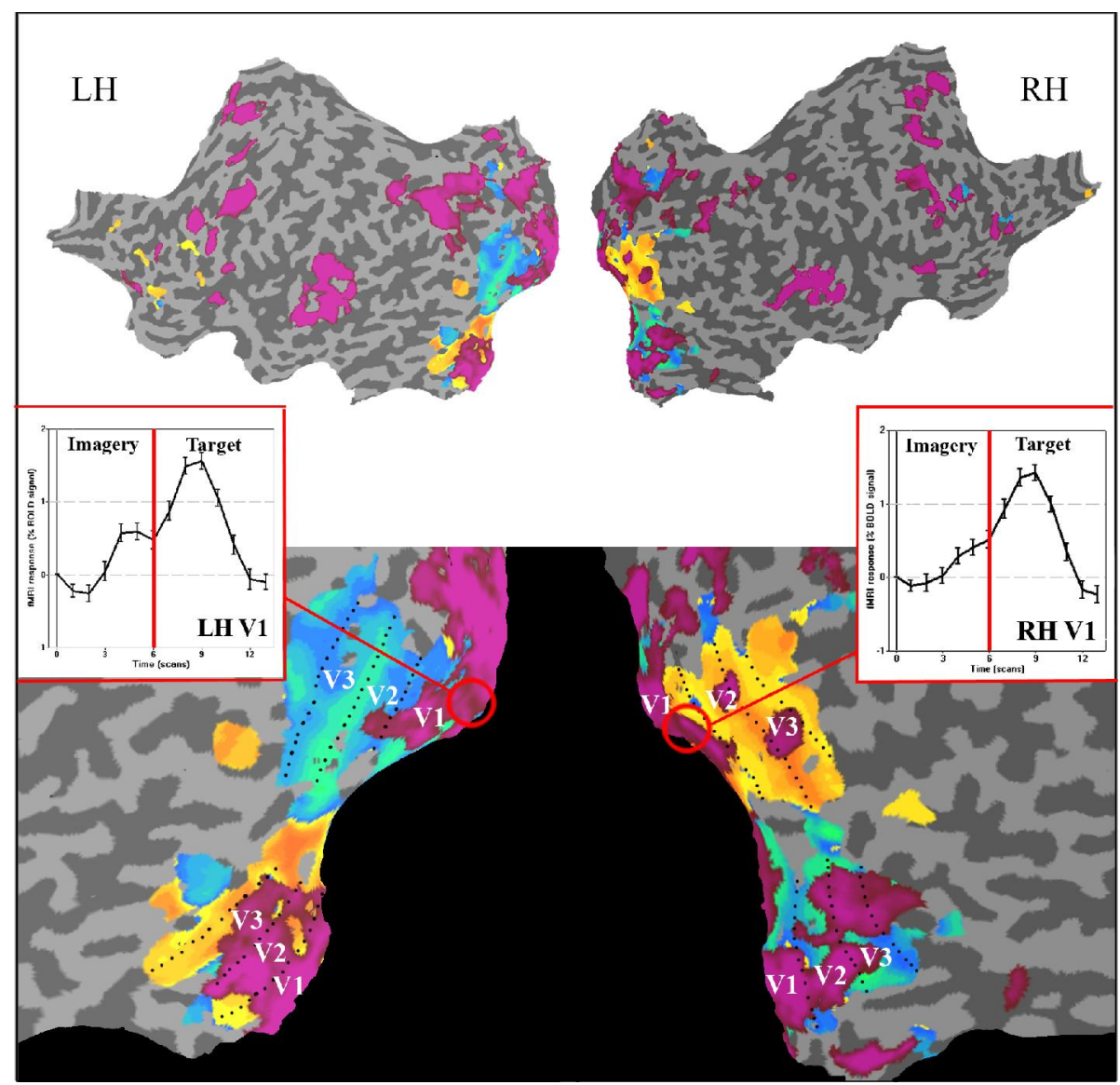

Figure 2.4 Single subject GLM data during imagery (purple) overlaid on this subjects' retinotopic map on a flattened representation of the cortical sheet. Event related average time courses in volumes of two regions of interest in primary visual cortex are presented on the left and right side of the figure.

\subsubsection{Fast versus slow reaction time trials}

In order to determine which regions, active during mental imagery, predict later performance on the task, we split the trials according to reaction times. The fMRI data median split in fast versus slow reaction time trials revealed a region in the mesial superior frontal gyrus that showed a significant difference for the tested contrast (341 voxels, $\mathrm{q}(\mathrm{FDR})<0.05)$. Figure 2.5 shows a larger BOLD signal in the mesial superior frontal gyrus for the slow trials compared to fast trials. 


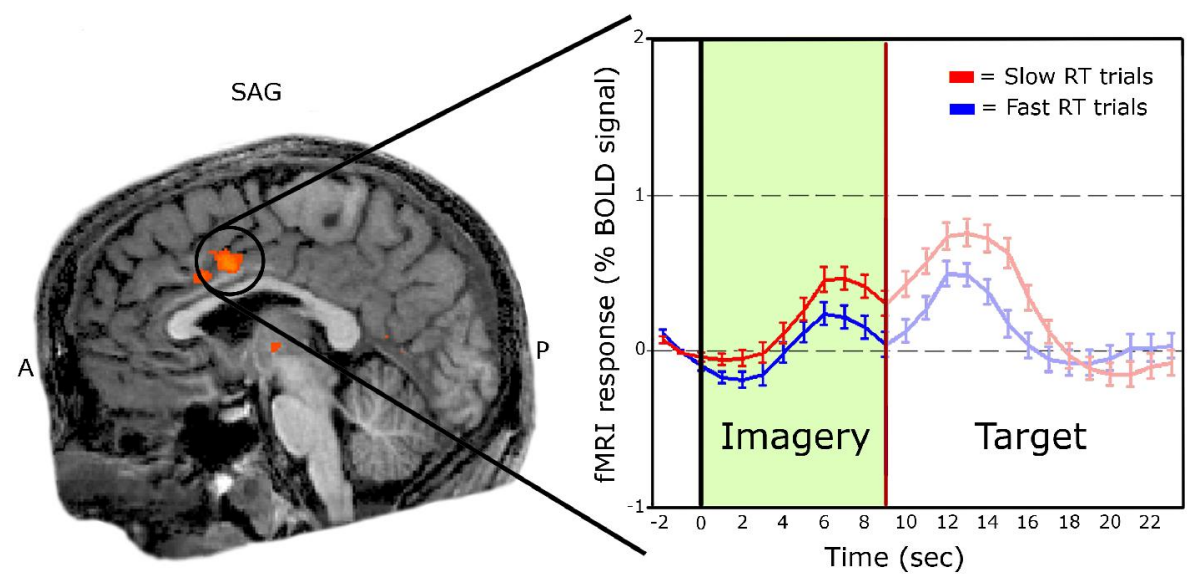

Figure 2.5 Results of multi-subject general linear model with a contrast of slow reaction time trials $>$ fast reaction time trials superimposed on T1-weighted data of a single subject, showing a region in the mesial superior frontal gyrus with enhanced response during slow reaction time trials $(\mathrm{q}(\mathrm{FDR})<0.05,341$ voxels). Right: Event-related average timecourses of this region in slow (red) and fast (blue) trials during imagery (before red line). 
Table 2.1 Significantly activated regions during scene imagery. L/R/m indicates whether the region was left or right lateralized or medially located.

\begin{tabular}{|c|c|c|c|c|c|}
\hline Region & $\mathrm{L} / \mathrm{R} / \mathrm{m}$ & Nr. voxels & $x$ & $\mathrm{y}$ & $\mathrm{z}$ \\
\hline \multicolumn{6}{|l|}{ Frontal } \\
\hline Middle frontal gyrus & $\mathrm{L}$ & 141 & -34 & 46 & 26 \\
\hline Middle frontal gyrus & $\mathrm{L}$ & 212 & -42 & 24 & 30 \\
\hline Middle frontal gyrus & $\mathrm{R}$ & 1011 & 32 & 39 & 30 \\
\hline Frontal eye fields & $\mathrm{L}$ & 577 & -41 & -2 & 32 \\
\hline Frontal eye fields & $\mathrm{R}$ & 2744 & 44 & 1 & 34 \\
\hline Premotor cortex & $\mathrm{L}$ & 4294 & -32 & -14 & 52 \\
\hline Premotor cortex & $\mathrm{R}$ & 3076 & 32 & -9 & 51 \\
\hline Superior frontal gyrus & $\mathrm{m}$ & 10020 & 1 & 4 & 46 \\
\hline Insula & $\mathrm{L}$ & 1588 & -31 & 16 & 9 \\
\hline Insula & $\mathrm{R}$ & 2523 & 34 & 18 & 8 \\
\hline \multicolumn{6}{|l|}{ Subcortical } \\
\hline Thalamus & $\mathrm{L}$ & 1338 & -10 & -17 & 8 \\
\hline Thalamus & $\mathrm{R}$ & 1306 & 9 & -14 & 9 \\
\hline Putamen & $\mathrm{L}$ & 592 & -16 & 10 & 3 \\
\hline Putamen & $\mathrm{R}$ & 1252 & 16 & 9 & 6 \\
\hline Red Nucleus & $\mathrm{L}$ & 222 & -5 & -23 & -3 \\
\hline Red Nucleus & $\mathrm{R}$ & 369 & 6 & -22 & -2 \\
\hline Pulvinar & $\mathrm{L}$ & 274 & -19 & -25 & 1 \\
\hline Pulvinar & $\mathrm{R}$ & 639 & 18 & -25 & 1 \\
\hline \multicolumn{6}{|l|}{ Temporal } \\
\hline Superior temporal gyrus & $\mathrm{L}$ & 13773 & 54 & -19 & 3 \\
\hline Superior temporal gyrus & $\mathrm{R}$ & 11863 & -52 & -21 & 7 \\
\hline Parahippocampal gyrus & $\mathrm{L}$ & 1937 & -28 & -35 & -14 \\
\hline Parahippocampal gyrus & $\mathrm{R}$ & 659 & 27 & -31 & -13 \\
\hline Inferior temporal gyrus & $\mathrm{L}$ & 553 & -46 & -52 & -11 \\
\hline \multicolumn{6}{|l|}{ Parietal } \\
\hline Superior parietal lobe & $\mathrm{L}$ & 11037 & -18 & -70 & 39 \\
\hline Superior parietal lobe & $\mathrm{R}$ & 9708 & 18 & -67 & 40 \\
\hline Intraparietal sulcus & $\mathrm{L}$ & 5237 & -35 & -47 & 38 \\
\hline Intraparietal sulcus & $\mathrm{R}$ & 9708 & 18 & -67 & 40 \\
\hline Precuneus & $\mathrm{L}$ & 1507 & -4 & -76 & 34 \\
\hline Precuneus & $\mathrm{R}$ & 1523 & 4 & -75 & 37 \\
\hline Parieto-occipital sulcus & $\mathrm{L}$ & 2017 & -17 & -65 & 17 \\
\hline Parieto-occipital sulcus & $\mathrm{R}$ & 3918 & 16 & -63 & 19 \\
\hline \multicolumn{6}{|l|}{ Occipital } \\
\hline Cuneus & $\mathrm{L}$ & 5557 & -6 & -77 & 8 \\
\hline Cuneus & $\mathrm{R}$ & 3283 & 4 & -78 & 13 \\
\hline Lingual gyrus & $\mathrm{L}$ & 8678 & -9 & -77 & -11 \\
\hline Lingual gyrus & $\mathrm{R}$ & 8435 & 10 & -78 & -9 \\
\hline Inferior occipital gyrus & $\mathrm{L}$ & 1356 & -24 & -85 & -2 \\
\hline Middle occipital gyrus & $\mathrm{L}$ & 3821 & -29 & -78 & 15 \\
\hline Calcarine sulcus & $\mathrm{m}$ & 14980 & 0 & -74 & 3 \\
\hline
\end{tabular}




\subsection{Discussion}

In this study we investigated the spatial properties of a functional network activated during scene imagery and the latency information of its nodes. We designed a task that enforced the mental analysis of objects and spatial layout of a scene and used complementary measurement and analyses techniques to capture the temporal and topographical aspects of this process.

In agreement with previous studies, the fMRI data showed task-related activity in regions of the ventral stream, the parahippocampal gyrus, and regions of the dorsal stream, e.g. the superior parietal lobe. Furthermore, we confirmed the involvement of several other regions, such as the mesial SFG, the right MFG, the premotor cortex, the calcarine sulcus and subcortical structures, stressing their fundamental role in visual mental imagery. Using BOLD latency mapping, we unravelled the temporal sequence of activation of these regions. We showed an early activation of the auditory regions and the subcortical structures, such as the thalamus and putamen. Furthermore, all frontal regions preceded the parietal and temporal regions in the onset of their activation. An additional latency difference was found between left and right superior parietal cortices.

Combining the fMRI data with the reaction time data revealed an interesting pattern of activation in the mesial SFG. When BOLD responses were high in mesial SFG during imagery this predicted slower reaction times on the task, while if BOLD responses were lower during imagery this was followed by faster reaction times.

\subsubsection{Frontal activity}

Several frontal regions were found to be active during this fMRI study. The frontal cortex is known to be involved in higher cognitive functions such as attention, memory and planning (Cabeza and Nyberg, 2000). During our mental imagery task these frontal regions most probably were engaged in memory retrieval, memory maintenance and the integration of different types of information into one visio- spatial mental picture, whilst communicating amongst each other and with regions in remote areas.

The mesial SFG seems to play a central role in these processes as can be inferred from its earlier onset during the task and its relevance in task performance. The mesial SFG includes parts of the (pre) SMA and anterior cingulate cortex, which are regions known to be involved in motor planning (Picard and Strick, 1996) and cognitive control (Critchley, 2005). As all analyses were performed on an imagery period with an average length of seven seconds, excluding the part of the trial during which the participant 
viewed the target, the mesial SFG activation is not likely to originate solely from response preparation and execution. The SFG has shown to be active in a variety of working memory tasks, and is here possibly involved in semantic processing of the auditory cues (Chee et al., 1999), spatial attention (Mitchell et al., 2000), integration of visual and spatial features (Mitchell et al., 2000; Prabhakaran et al., 2000; Munk et al., 2002), and executive function (D'Esposito et al., 1995; Talati and Hirsch, 2005). Especially the latter two functions seem to be essential as supported by the relationship that was found between the fMRI data and the reaction times. A high BOLD response in mesial SFG during imagery predicted slower reaction times on the task, while if BOLD responses were lower during imagery this was followed by faster reaction times. Studies often interpreted a higher BOLD signal in this region during conditions with a high workload and longer reaction times as a sign of more cognitive control, or moderating activity (D'Esposito et al., 1995; Linden et al., 2003; Rypma and Prabhakaran, 2009). Inhibitory interneurons could play a part in this process, by streamlining neuronal activity. One could theorize that during trials in which the participant found it difficult to imagine the scene, more interneurons were addressed to inhibit irrelevant input, leading to more oxygen consumption and a higher BOLD signal (Swadlow, 2003; Buzsaki et al., 2007; Ekstrom, 2010).

Two other frontal regions were engaged during the imagery of scenes. The right medial frontal gyrus seems to be involved in retrieval of the memorized scenes (Buckner et al., 1996; Tulving et al., 1996). As suggested by previous research, the right frontal gyrus might communicate with the parietal cortex and subcortical structures in order to retrieve the scene (Tulving et al., 1996). The premotor cortex, like the mesial SFG, is also often activated during response preparation. Due to aforementioned reasons, the premotor cortex is also likely to be involved in cognitive processing during this task, rather than solely motor preparation. As described in Mellet et al. (1996), the premotor cortex plays a role in visuospatial tasks. They suggested that the parietal and the frontal lobes work together in deciphering a spatial (mental) environment. Furthermore, the premotor cortex could be involved in internally guided shifts of attention (Rosen et al., 1999; Mitchell et al., 2000), while the subjects mentally build the scene from the different objects.

Overall, due to its early onset during task performance, the frontal regions seem to orchestrate the activity of the remote visual regions in temporal and parietal cortex by means of top-down influence and bidirectional feedback, in order to encode and retain an integrated visuo-spatial mental picture. 


\subsubsection{Parietal activity}

The parietal cortex is known to be involved in a large variety of mental imagery tasks. The largest regions of parietal activation during this task are located in the superior parietal lobe (SPL), the precuneus and the intraparietal sulcus (IPS). The SPL and IPS have been shown to be active during different types of visuospatial imagery tasks (e.g. Alivisatos and Petrides, 1997; Goebel et al., 1998; Mellet et al., 2000a; Trojano et al., 2000; Formisano et al., 2002; Podzebenko et al., 2002; Sack et al., 2002; Trojano et al., 2002; Sack et al., 2005; Phillips et al., 2009). This study required participants to imagine e.g. the spatial lay-out of a scene, therefore the activation in SPL and IPS is likely to reflect processing of the spatial properties of the mental image. A more specific proposition on the functional role of these regions can be based on the results of the latency mapping analysis. The latency mapping analysis revealed a substantial latency difference between left and right superior parietal cortices. Previous research has found temporal and functional differences between left and right posterior parietal cortex during visuospatial imagery tasks (Formisano et al., 2002; Sack et al., 2002; Sack et al., 2005). In this study, we also find an earlier activation of left SPL compared to right SPL. This latency difference might reflect divergent functional specializations, in which the left superior parietal lobe could be more involved in construction of the spatial relations, and the right superior parietal lobe in visuospatial image integration and inspection.

The precuneus is highly connected with the mesial SFG, thalamus and other parietal regions (Cavanna and Trimble, 2006). This multifaceted region seems to be involved in retrieval of spatial memory content (Daselaar et al., 2009), attentional shifts (without eye movements), in conjunction with the premotor cortex, and in visuospatial processing of the mental image (Hanakawa et al., 2003; Cavanna and Trimble, 2006).

\subsubsection{Temporal activity}

Concurrent with the validation of the parietal activation due to the spatial properties of the mental image, our results confirm the involvement of category specific areas in the inferior temporal lobe during scene imagery. Similar to the results of O'Craven \& Kanwisher (2000), we found a relatively smaller increase of BOLD amplitude in the parahippocampal gyrus during scene imagery than during the perception of the target. The parahippocampal gyrus (PHG) is here most likely involved in memory retrieval (Hayes et al., 2007; Prince et al., 2009). Like the SPL and the precuneus, the PHG is also likely to convey information to the prefrontal cortex, where information is integrated. 
This idea is supported by the finding of projections from the parahippocampal gyrus to the medial prefrontal cortex in rhesus monkeys (Bachevalier et al., 1997). Thus, the parietal and temporal regions both seem to communicate with the prefrontal cortex, rather than between them, in order to create a complete mental scene.

\subsubsection{Occipital activity}

Rather surprisingly, we also found activation of early visual cortex during imagery. The involvement of early visual cortex during mental imagery is still debated as some studies reported activation (Le Bihan et al., 1993; Kosslyn et al., 1999; Klein et al., 2004), while others found no such evidence (D'Esposito et al., 1997; Knauff et al., 2000). During previous studies early visual cortex activity was mostly elicited by object imagery tasks and not by spatial imagery tasks (Kosslyn and Thompson, 2003), therefore we did not necessarily expect the imagery of scenes to drive early visual cortex. The fact that this task did activate early visual cortex most likely occurred because the task required forming a detailed representation of the realistic mental image, including objects. Detailed image inspection is, according to Kosslyn \& Thompson's meta-analysis (2003), another possible prerequisite of early visual cortex activation during imagery. It is likely that the early visual cortex works closely together with category-specific regions in ventro-temporal cortex in order to encode the visually detailed objects and then conveys this information to mesial SFG for integrating and maintaining a scene representation.

\subsubsection{Conclusions}

This study set out to investigate the topography and latency information of the functional nodes of a full-brain network that integrates object and spatial information into the representation of a visual mental scene. We found an earlier onset of frontal regions and subcortical structures compared to ventro-temporal and parietal regions. These early frontal regions work closely together though with the remote parietal regions to encode the spatial sketch of the scene and with occipital-temporal regions to encode the detailed visual representations of the objects. Subsequently, mesial SFG is likely to integrate and maintain the incoming information from the different remote regions. The importance of this controlling role of the mesial SFG is further confirmed by its influence on task performance. 
Alivisatos B, Petrides M (1997) Functional activation of the human brain during mental rotation. Neuropsychologia 35:111-118.

Bachevalier J, Meunier M, Lu MX, Ungerleider LG (1997) Thalamic and temporal cortex input to medial prefrontal cortex in rhesus monkeys. Exp Brain Res 115:430-444.

Barton JJ, Cherkasova M (2003) Face imagery and its relation to perception and covert recognition in prosopagnosia. Neurology 61:220-225.

Been M, Jans B, De Weerd P (in preparation) fMRI reveals learning dependent and contrast specific increase in bold response.

Buckner RL, Raichle ME, Miezin FM, Petersen SE (1996) Functional anatomic studies of memory retrieval for auditory words and visual pictures. Journal of Neuroscience 16:6219-6235.

Buzsaki G, Kaila K, Raichle M (2007) Inhibition and brain work. Neuron 56:771-783.

Cabeza R, Nyberg L (2000) Imaging cognition II: An empirical review of 275 PET and fMRI studies. J Cogn Neurosci 12:1-47.

Cavanna AE, Trimble MR (2006) The precuneus: a review of its functional anatomy and behavioural correlates. Brain 129:564-583.

Chee MW, O'Craven KM, Bergida R, Rosen BR, Savoy RL (1999) Auditory and visual word processing studied with fMRI. Human Brain Mapping 7:15-28.

Critchley HD (2005) Neural mechanisms of autonomic, affective, and cognitive integration. J Comp Neurol 493:154-166.

D'Esposito M, Detre JA, Alsop DC, Shin RK, Atlas S, Grossman M (1995) The neural basis of the central executive system of working memory. Nature 378:279-281.

D'Esposito M, Detre JA, Aguirre GK, Stallcup M, Alsop DC, Tippet LJ, Farah MJ (1997) A functional MRI study of mental image generation. Neuropsychologia 35:725-730.

Daselaar SM, Prince SE, Dennis NA, Hayes SM, Kim H, Cabeza R (2009) Posterior Midline and Ventral Parietal Activity is Associated with Retrieval Success and Encoding Failure. Front Hum Neurosci 3:13.

Denis M, Logie RH, Cornoldi C, de Vega M, Engelkamp J (2001) Imagery, language and visuospatial thinking. East Sussex: Psychology Press Ltd.

Ekstrom A (2010) How and when the fMRI BOLD signal relates to underlying neural activity: the danger in dissociation. Brain Res Rev 62:233-244.

Farah MJ (1984) The neurological basis of mental imagery: A componential analysis. Cognition 18:245.

Finke RA (1985) Theories relating mental imagery to perception. Psychological Bulletin 98:236.

Formisano E, Linden DE, Di Salle F, Trojano L, Esposito F, Sack AT, Grossi D, Zanella FE, Goebel R (2002) Tracking the mind's image in the brain I: time-resolved fMRI during visuospatial mental imagery. Neuron 35:185-194.

Ganis G, Thompson WL, Kosslyn SM (2004) Brain areas underlying visual mental imagery and visual perception: An fMRI study. Cognitive Brain Research 20:226.

Genovese CR, Lazar NA, Nichols T (2002) Thresholding of statistical maps in functional neuroimaging using the false discovery rate. Neuroimage 15:870-878. 
Goebel R, Esposito F, Formisano E (2006) Analysis of functional image analysis contest (FIAC) data with brainvoyager QX: From single-subject to cortically aligned group general linear model analysis and self-organizing group independent component analysis. Human Brain Mapping 27:392-401.

Goebel R, Linden DEJ, Lanfermann H, Zanella FE, Singer W (1998) Functional imaging of mirror and inverse reading reveals separate coactivated networks for oculomotion and spatial transformations. Neuroreport: An International Journal for the Rapid Communication of Research in Neuroscience 9:713.

Hanakawa T, Immisch I, Toma K, Dimyan MA, Van Gelderen P, Hallett M (2003) Functional properties of brain areas associated with motor execution and imagery. J Neurophysiol 89:989-1002.

Hayes SM, Nadel L, Ryan L (2007) The effect of scene context on episodic object recognition: parahippocampal cortex mediates memory encoding and retrieval success. Hippocampus 17:873-889.

Ishai A, Ungerleider LG, Haxby JV (2000) Distributed neural systems for the generation of visual images. Neuron 28:979-990.

Ishai A, Haxby JV, Ungerleider LG (2002) Visual imagery of famous faces: effects of memory and attention revealed by fMRI. Neuroimage 17:1729-1741.

Klein I, Dubois J, Mangin JF, Kherif F, Flandin G, Poline JB, Denis M, Kosslyn SM, Le Bihan D (2004) Retinotopic organization of visual mental images as revealed by functional magnetic resonance imaging. Brain Res Cogn Brain Res 22:26-31.

Knauff M, Kassubek J, Mulack T, Greenlee MW (2000) Cortical activation evoked by visual mental imagery as measured by fMRI. Neuroreport 11:3957-3962.

Kosslyn SM, Thompson WL (2003) When is early visual cortex activated during visual mental imagery? Psychol Bull 129:723-746.

Kosslyn SM, Thompson WL, Alpert NM (1997) Neural systems shared by visual imagery and visual perception: a positron emission tomography study. Neuroimage 6:320-334.

Kosslyn SM, Kosslyn SM, Reiser BJ, Farah MJ, Fliegel SL (1983) Generating visual images: Units and relations. Journal of Experimental Psychology: General 112:278.

Kosslyn SM, Pascual-Leone A, Felician O, Camposano S, Keenan JP, Thompson WL, Ganis G, Sukel KE, Alpert NM (1999) The role of area 17 in visual imagery: convergent evidence from PET and rTMS. Science 284:167-170.

Le Bihan D, Turner R, Zeffiro TA, Cuenod CA, Jezzard P, Bonnerot V (1993) Activation of human primary visual cortex during visual recall: a magnetic resonance imaging study. Proc Natl Acad Sci U S A 90:11802-11805.

Linden DE, Bittner RA, Muckli L, Waltz JA, Kriegeskorte N, Goebel R, Singer W, Munk MH (2003) Cortical capacity constraints for visual working memory: dissociation of fMRI load effects in a fronto-parietal network. Neuroimage 20:1518-1530.

Mechelli A, Price CJ, Friston KJ, Ishai A (2004) Where bottom-up meets top-down: neuronal interactions during perception and imagery. Cerebral Cortex 14:1256-1265. 
Mellet E, Tzourio N, Crivello F, Joliot M, Denis M, Mazoyer B (1996) Functional anatomy of spatial mental imagery generated from verbal instructions. Journal of Neuroscience 16:6504-6512.

Mellet E, Tzourio-Mazoyer N, Bricogne S, Mazoyer B, Kosslyn SM, Denis M (2000a) Functional anatomy of high-resolution visual mental imagery. Journal of Cognitive Neuroscience 12:98-109.

Mellet E, Briscogne S, Tzourio-Mazoyer N, Ghaem O, Petit L, Zago L, Etard O, Berthoz A, Mazoyer B, Denis M (2000b) Neural correlates of topographic mental exploration: the impact of route versus survey perspective learning. Neuroimage 12:588-600.

Mitchell KJ, Johnson MK, Raye CL, D'Esposito M (2000) fMRI evidence of age-related hippocampal dysfunction in feature binding in working memory. Cognitive Brain Research 10:197-206.

Munk MH, Linden DE, Muckli L, Lanfermann H, Zanella FE, Singer W, Goebel R (2002) Distributed cortical systems in visual short-term memory revealed by event-related functional magnetic resonance imaging. Cerebral Cortex 12:866-876.

O'Craven KM, Kanwisher N (2000) Mental imagery of faces and places activates corresponding stiimulus-specific brain regions. Journal of Cognitive Neuroscience 12:1013-1023.

Phillips JS, Velanova K, Wolk DA, Wheeler ME (2009) Left posterior parietal cortex participates in both task preparation and episodic retrieval. Neuroimage 46:1209-1221.

Picard N, Strick PL (1996) Motor areas of the medial wall: a review of their location and functional activation. Cereb Cortex 6:342-353.

Podzebenko K, Egan GF, Watson JD (2002) Widespread dorsal stream activation during a parametric mental rotation task, revealed with functional magnetic resonance imaging. Neuroimage 15:547-558.

Prabhakaran V, Narayanan K, Zhao Z, Gabrieli JD (2000) Integration of diverse information in working memory within the frontal lobe. Nature Neuroscience 3:85-90.

Prince SE, Dennis NA, Cabeza R (2009) Encoding and retrieving faces and places: distinguishing process- and stimulus-specific differences in brain activity. Neuropsychologia 47:22822289.

Richter W, Somorjai R, Summers R, Jarmasz M, Menon RS, Gati JS, Georgopoulos AP, Tegeler C, Ugurbil K, Kim SG (2000) Motor area activity during mental rotation studied by timeresolved single-trial fMRI. Journal of Cognitive Neuroscience 12:310-320.

Rosen AC, Rao SM, Caffarra P, Scaglioni A, Bobholz JA, Woodley SJ, Hammeke TA, Cunningham JM, Prieto TE, Binder JR (1999) Neural basis of endogenous and exogenous spatial orienting. A functional MRI study. Journal of Cognitive Neuroscience 11:135-152.

Rypma B, Prabhakaran V (2009) When less is more and when more is more: The mediating roles of capacity and speed in brain-behavior efficiency. Intelligence 37:207-222.

Sack AT, Camprodon JA, Pascual-Leone A, Goebel R (2005) The dynamics of interhemispheric compensatory processes in mental imagery. Science 308:702-704.

Sack AT, Sperling JM, Prvulovic D, Formisano E, Goebel R, Di Salle F, Dierks T, Linden DE (2002) Tracking the mind's image in the brain II: transcranial magnetic stimulation reveals parietal asymmetry in visuospatial imagery. Neuron 35:195-204. 
Sereno MI, Dale AM, Reppas JB, Kwong KK, Belliveau JW, Brady TJ, Rosen BR, Tootell RB (1995) Borders of multiple visual areas in humans revealed by functional magnetic resonance imaging. Science 268:889-893.

Shepard RN, Metzler J (1971) Mental rotation of three-dimensional objects. Science 171:701.

Swadlow HA (2003) Fast-spike interneurons and feedforward inhibition in awake sensory neocortex. Cereb Cortex 13:25-32.

Talairach J, Tournoux P (1988) Co-planar Stereotaxic Atlas of the Human Brain: 3-Dimensional Proportional System - an Approach to Cerebral Imaging. New York: Thieme Medical Publishers.

Talati A, Hirsch J (2005) Functional specialization within the medial frontal gyrus for perceptual go/no-go decisions based on "what," "when," and "where" related information: an fMRI study. Journal of Cognitive Neuroscience 17:981-993.

Thompson WL, Kosslyn SM, Sukel KE, Alpert NM (2001) Mental imagery of high- and lowresolution gratings activates area 17 . Neuroimage 14:454-464.

Trojano L, Grossi D (1994) A critical review of mental imagery defects. Brain and Cognition 24:213.

Trojano L, Trojano L, Linden DEJ, Formisano E, Goebel R, Sack AT, Di Salle F (2004) What clocks tell us about the neural correlates of spatial imagery. European Journal of Cognitive Psychology 16:653.

Trojano L, Grossi D, Linden DE, Formisano E, Hacker H, Zanella FE, Goebel R, Di Salle F (2000) Matching two imagined clocks: the functional anatomy of spatial analysis in the absence of visual stimulation. Cerebral Cortex 10:473-481.

Trojano L, Grossi D, Linden DE, Formisano E, Goebel R, Cirillo S, Elefante R, Di Salle F (2002) Coordinate and categorical judgements in spatial imagery. An fMRI study. Neuropsychologia 40:1666-1674.

Tulving E, Markowitsch HJ, Craik FE, Habib R, Houle S (1996) Novelty and familiarity activations in PET studies of memory encoding and retrieval. Cerebral Cortex 6:71-79.

Warnking J, Dojat M, Guerin-Dugue A, Delon-Martin C, Olympieff S, Richard N, Chehikian A, Segebarth C (2002) fMRI retinotopic mapping--step by step. Neuroimage 17:1665-1683. 


\section{Chapter 3}

\section{Investigating the spectro-temporal brain dynamics of scene imagery}

\section{Abstract}

In this electroencephalography (EEG) study we investigated the spectro-temporal properties of the brain network underlying the imagery of natural scenes. We strived to uncover which frequency bands are involved during a task that required imagining objects and their spatial lay-out, the integration of these features into a scene, and its subsequent maintenance in memory. During the imagery task we found theta and beta synchronization in central and right anterior sites and beta and alpha de-synchronization in left and right posterior sites. We found no consistent oscillatory activity at ventro-temporal or occipital sites. The results of our study suggest that (spatial) memory retrieval during scene imagery is supported by synchronization of theta oscillations in right frontal regions, together with de-synchronization in bilateral parietal sites. Theta and beta synchronization in a midline frontal region seems to underlie the integration of information into one visual scene. The overall mental imagery process seems to be supported by alpha de-synchronization in posterior sites, which might reflect global processes, such as attention or the inhibition of task non-relevant regions.

\section{Based on}

A.W. de Borst, A.T. Sack, B.M. Jansma, F. Esposito, F. de Martino, G. Valente, A. Roebroeck, F. di Salle, R. Goebel, and E. Formisano. Integration of "what" and "where in frontal cortex during visual imagery of scenes. (submitted). 


\subsection{Introduction}

In this study we examine the (spectro-) temporal properties of brain activity during visual mental imagery by means of electroencephalography (EEG), a recording technique with high temporal resolution. So far, only a few EEG (or magnetoencephalography [MEG]) studies have focussed on visual mental imagery specifically (Barrett and Ehrlichman, 1982; Farah et al., 1988; Lehmann et al., 1993; Schupp et al., 1994; Marks and Isaac, 1995; Petsche et al., 1997; Cooper et al., 2003; Ganis and Schendan, 2008). On the contrary, there have been many studies using the same techniques to investigate visual working memory. As visual working memory and visual mental imagery share a great deal of mental operations (see Chapter 1), findings from both these research fields are of interest here and are shortly reviewed below.

Mental imagery and working memory are internally generated processes and their onset cannot be precisely linked to a specific stimulus. Therefore, standard EEGMEG analyses that average the data according to a certain stimulus onset, resulting in an event related potential (ERP) signal, are less suitable for this type of research. Instead, many studies have focussed on analyzing (non stimulus locked) oscillations in the frequency bands common to the human brain: delta $[<4 \mathrm{~Hz}]$, theta $[4-7 \mathrm{~Hz}]$, alpha [8 - $12 \mathrm{~Hz}]$, beta $[12-30 \mathrm{~Hz}]$ and gamma [> $30 \mathrm{~Hz}$. These oscillations in different frequency bands are suggested to relate to specific cognitive functions, depending on its location in the brain.

One of the most reported frequency bands during mental imagery is the alpha frequency. Many EEG studies reported a suppression of neuronal oscillatory responses (de-synchronization) in the alpha frequency range at posterior and frontal sites during visual mental imagery compared to a baseline task. This de-synchronization is interpreted as a reflection of (imagery-related) cognitive engagement of these regions (Barrett and Ehrlichman, 1982; Petsche et al., 1992; Marks and Isaac, 1995; Petsche et al., 1997; Gill et al., 1998; Nikolaev and Anokhin, 1998; Bhattacharya and Petsche, 2005). Oppositely, an increase in alpha oscillations (synchronization) has recently been suggested to reflect the inhibition of sensory input during a mental task, or more generally, inhibition in task- irrelevant areas during high demand information processing (Klimesch et al., 2000; Cooper et al., 2003). Both processes (alpha synchronization and de-synchronization) may occur during the same task in inhibited and engaged regions (Klimesch et al., 2000). The presence of alpha oscillations during mental imagery thus seems to reflect a general task-engagement effect, which is particularly visible during mental tasks, as all sensory input needs to be suppressed.

When performing a mental imagery task, information is usually recalled from 
memory and subsequently held and manipulated in working memory. The theta frequency band and its synchronization with the gamma frequency band seem to play a crucial role during working memory. Reported increases in theta band power (synchronization) at frontal sites most likely reflect working memory processes during mental imagery tasks (Rugg and Venables, 1980; Petsche et al., 1997; Bhattacharya and Petsche, 2005). Theta band oscillations are often linked to beta and gamma oscillations that are related to the content of the memory representation.

Comparable to the results of neuroimaging studies into visual mental imagery, as described in Chapter 2.1, some evidence for corresponding neural processes between perception and mental imagery, specifically the ventral "what" and the dorsal "where" processing streams, has been gathered by EEGMEG studies. Studies of auditory working memory revealed gamma band increases in the ventral and dorsal stream during MEG measurements of pattern and spatial auditory working memory tasks (for a review see Kaiser and Lutzenberger, 2003). Also in visual mental imagery research, EEGMEG effects localized to ventral and dorsal processing streams have been reported for object and spatial imagery, respectively. For instance, Pegna et al. (1997) have shown that during mental rotation an ERP segment, localized to the right parieto-occipital cortex, increased in duration with rotation angle. More recently, Holz et al. (2010) also reported enhanced phase-synchronisation between theta and high gamma $(50-70 \mathrm{~Hz})$ oscillations during visual spatial working memory in the parieto-occipital cortex. On the other hand, for object imagery, Tallon-Baudry et al. (1998) showed a sustained gamma increase at occipito-temporal and fronto-central electrodes during the delay of a matchto-sample task. Another effect in the ventral stream related to object imagery was found by Ganis and Schendan (2008) during an adaptation paradigm involving the imagery and perception of faces. Visual adapters decreased the amplitude of the N170 in the fusiform gyrus, while visualized adaptors enhanced its amplitude. This suggests that top-down reactivation of the fusiform gyrus by visual mental imagery, oppositely to perception, enhances processing of the following stimulus. In sum, although not many MEG/EEG studies have consistently investigated spatial or object mental imagery, the available evidence seems to suggest that mental imagery of objects and spatial configurations is reflected in gamma synchronization in the ventral and dorsal pathway and can have stimulus-specific and localized effects on the ERP.

In the current study we systematically investigate the spectro-temporal dynamics of the neuronal oscillations underlying the performance of a behaviourally-controlled visual scene imagery task. With our task we hope to uncover the oscillations patterns related to object and spatial imagery, as well as to memory maintenance. Based on our fMRI results, we expect to find multiple oscillatory sources in ventral occipital and 
dorsal parietal cortex, reflecting respectively the object and spatial components of our task. Previous EEGMEG studies indicate the alpha and the gamma bands as frequency of main interest (although gamma band oscillations have been mostly reported with MEG). Furthermore, we expect multiple frontal components, reflecting the working memory related processes (right-lateralized, in theta frequency range) and the processes of coordination and orchestration (central distribution) as suggested by previous fMRI results on the role of the frontal cortex in mental imagery (Chapter 2). 


\subsection{Methods}

\subsubsection{Participants}

Seven healthy right-handed volunteers (3 female, mean age 24.3 years; range 21-29) participated in the EEG study. All participants had normal or corrected-to-normal vision. The local ethical committee approved the studies and all participants gave their written informed consent.

\subsubsection{Stimuli and task}

Twenty-seven digital colour photographs were used as stimuli (screen and picture resolution 1024 by 768 pixels). Three photographs (see example in Figure 3.1, for colour version see Figure 2.1) depicted the interior of a room and comprised several pieces of furniture. These photographs defined the visual imagery content and were solely used during a training phase. Twelve photographs (targets) were fragments of the three original scenes (four per scene), containing a minimum of two partly visible objects. The other twelve photographs (targets) were these same images mirrored across the horizontal axis (see Figure 3.1). The default image was a grey background ( $50 \%$ black, $50 \%$ white). All photographs contained a centralized white fixation cross and were presented at 5 by $7^{\circ}$ visual angle. A smaller visual angle than during the fMRI experiment (Chapter 2) was chosen to minimize eye movement artefacts in the EEG.

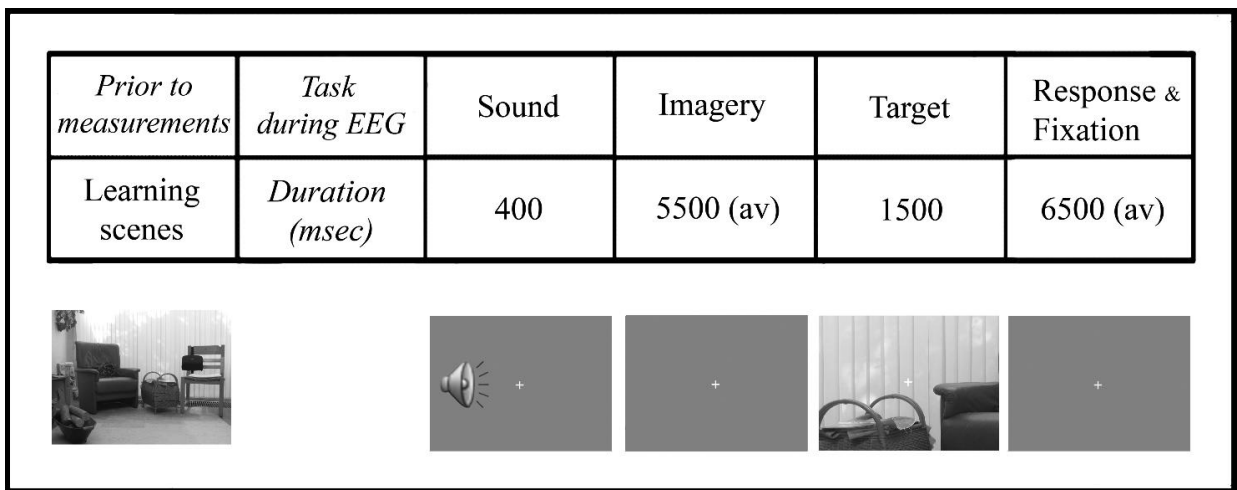

Figure 3.1 Schematic overview of the temporal course of one exemplary trial in the EEG experiment.

The EEG experiment consisted of 216 (3 associations $x 72$ repetitions) trials. As depicted 
in Figure 3.1, each trial of the experiment started with an auditory cue. In the EEG experiment tones were used instead of spoken cues (Chapter 2), because their EEG patterns are more similar. The three auditory stimuli were $400 \mathrm{~ms}$ tones at 800, 1000 and $1200 \mathrm{~Hz}$. During the subsequent interval participants imagined the scene that they previously had learned to associate with the cue. After a variable delay $(5,5.5$, or $6 \mathrm{~s}$, average $=5.5 \mathrm{~s}$ ) a mirrored or non-mirrored fragment of the scene (50-50\% distribution) was visually presented as a target for $1.5 \mathrm{~s}$. After each target participants pressed a button with their right middle or index finger to indicate whether the fragment matched the imagined scene, or was mirrored (reversed in half of the participants). All trials ended with a fixation period (4-5s) during which the participants performed no task. The variable delay and fixation period were shortened compared to the fMRI experiment (Chapter 2) because no hemodynamic delay had to be taken into account. The interval lengths were pseudo-randomized over trials and the occurrence of each period length was equally distributed over the three types of trials (scene one, two and three) and type of target picture. The trials (e.g. the order of the tones and target pictures) were pseudorandomized.

Prior to the EEG main experiment, participants were trained on imagining three scenes, associated with three auditory cues. The training session consisted of 24 randomized trials of simultaneous presentation of the auditory stimulus and the corresponding visual scene ( $5 \mathrm{~s})$, followed by a fixation period (6s) during which the participants imagined the scene. After training, participants received task instructions and were asked to vividly visualize the correct scene after each cue in the main experiment, while fixating at the fixation cross.

The training session and the main experiment were preceded by an auditory control task. During this task participants were presented with a total of 216 randomized trials of three auditory stimuli, to which they listened passively. Each trial consisted of a $400 \mathrm{~ms}$ tone and a fixation period (random interval between 6 and $7 \mathrm{~s}$ ). These same tones were used as cues in the training session and the main experiment.

\subsubsection{Data acquisition}

The EEG was recorded in a sound-attenuating and electrically shielded room from 64 electrode sites (EasyCap system, Equidistant 61-Channel Arrangement cap). One electrode was placed under the left eye to measure eye movements. The reference was located behind the left ear and the ground on the right cheek. All impedances were below $5 \mathrm{k} \Omega$. A chin rest was used to minimize head motion and to keep the head at a 
fixed distance from the PC screen to maintain the correct visual angle. The data were sampled at $500 \mathrm{~Hz}$, with a band-pass filter of $0.01-100 \mathrm{~Hz}$.

\subsubsection{Data analyses}

An open source Matlab toolbox, EEGLAB (Delorme and Makeig, 2004), was used for EEG data processing. The data were downsampled to $250 \mathrm{~Hz}$ for processing purposes and band-pass filtered between 0.5 and $100 \mathrm{~Hz}$. Three separate datasets were created for the auditory control task, the practice session and the main experiment. The data of the practice session were not used for the subsequent analyses. Ground and reference electrodes were removed from the datasets and the datasets were re-referenced to an average reference. All data were epoched from -2000 to $4000 \mathrm{~ms}$ relative to auditory stimulus onset, including a baseline $(-2000-0 \mathrm{~ms})$. For the control condition these epochs contained the auditory stimulus and a silent period. For the imagery condition these epochs contained the auditory stimulus and the mental imagery of the scenes, excluding target picture and response. Channels with a high noise level were removed (five channels on average). Eye movement artefacts were removed using an amplitude cut-off of $90 \mathrm{mV}$. Residual eye movements and muscular artefacts were manually removed. In participants with a high number of eye movements, artefact rejection was accomplished by performing an independent component analysis (ICA) and removing the eye movement related components (Jung et al., 2000).

The pre-processed epochs of all participants were then subjected to a temporal ICA analysis (extended infomax, Makeig et al., 2004). An approximately equal number of components (depending on the final number of accepted channels) were extracted for each participant, on average 58 for the auditory control task and 57 for the main experiment. The activity time courses of these components were further processed using time-frequency decompositions.

\section{Time-frequency analyses}

Time-frequency decompositions were performed on the trials of the independent components. Event related spectral perturbation (ERSP) and inter-trial coherence (ITC) plots were calculated for the components of each condition and participant separately (Makeig et al., 2004). ERSP plots represent the changes in spectral power over a time window, compared to a baseline time window, averaged over trials. Here, $-2000 \mathrm{~ms}$ till 0 
ms before auditory cue onset were used as baseline and the first $4000 \mathrm{~ms}$ of the imagery period were targeted for analyses (0-4000 ms after cue onset). Initial inspection showed no clear-cut spectral power changes in the gamma frequency [30 - 100Hz], therefore Morlet wavelets (window of 209 samples $(836 \mathrm{~ms})$ ) were used to extract spectral power changes in the frequency range [1 - $40 \mathrm{~Hz}$. A total of 4000 points (40 frequency bins and 100 temporal bins) were estimated in the selected time-frequency window. Five relevant ICs were selected based on across-subject consistency of topographic maps, spectral power plots and time- frequency content. These components were labelled: "Central", "Left Posterior", "Right Posterior", "Right Anterior", and "Central Anterior" (see Figure 3.2). Averaged event-related spectral power for each subject and component (linear scale, baseline normalized) within four time-frequency bands (frequencies: theta [4 - 7 $\mathrm{Hz}$ ], alpha [8 - $12 \mathrm{~Hz}$ ], low beta [12 - $15 \mathrm{~Hz}$ ] and high beta [15 - 30 Hz], 100 temporal bins) was used to perform statistical analysis between the auditory control task and the imagery task (paired t-test, $\mathrm{N}=7$ ).

\section{Reaction time based time-frequency analyses}

For each participant, reaction times (onset time button-press minus onset time target) of the correct trials were calculated. Trials were ordered and a median split was applied, resulting in a group of "fast" and "slow" trials per participant. EEG trials were labelled based on these two categories and corresponding epochs were selected and grouped. For each of the five components, averaged event related spectral power (over trials) (linear scale, baseline normalized) within three time-frequency bands (frequencies: theta [4 - 7 $\mathrm{Hz}$, alpha [8 - $12 \mathrm{~Hz}]$ and beta [12 - $30 \mathrm{~Hz}], 100$ temporal bins) was calculated per participant and used to perform statistical analysis between the imagery epochs that were either followed by fast or slow reaction times (paired t-test, $N=7$ ). 


\subsection{Results}

All EEG results are solely based on the imagery period during the trials, excluding target picture information.

\subsubsection{Behavioural results}

The participants $(\mathrm{N}=7)$ responded correctly on 91.1 percent $(\mathrm{SD}=4.65)$ of the nonmirrored trials and 90.6 percent $(\mathrm{SD}=4.06)$ of the mirrored trials with an average reaction time $1366 \mathrm{~ms}(\mathrm{SD}=588.2)$ for non-mirrored and $1483 \mathrm{~ms}(\mathrm{SD}=512.9)$ for mirrored trials. A paired sampled t-test showed that responses were significantly faster $(\mathrm{t}(6)=-3.349, \mathrm{p}<0.015)$ during the non-mirrored trials.

\subsubsection{Independent Component \& time-frequency analyses}

Figure 3.2 shows the average event related spectral perturbation (ERSP) plots of the five selected components in the control condition and the imagery condition. The results from the paired t-tests revealed no significant difference at any time-point within the [1$15 \mathrm{~Hz}$ ] frequency range for the central independent component (IC) ( $p>0.05$ ). This component most likely reflects auditory processing of the cue, which is identical for the control and mental imagery task. The posterior and anterior ICs all showed an extensive change in spectral power during the imagery condition compared to the control condition. Left and right posterior ICs showed a larger decrease in spectral power (desynchronisation) in the alpha and beta band during the imagery condition. They were significantly different $(\mathrm{p}<0.05)$ between conditions in the alpha [8-12 Hz] (displayed) and beta [12-30 Hz] (similar results, not displayed) frequency bands in large time windows (see Figure 3.2, Box Average, green lines). Similar to the fMRI results of Chapter 2, the spectral power during mental imagery showed an earlier significant divergence from the control condition in the left posterior IC compared to the right posterior IC. The right anterior IC and the central anterior IC showed extensive spectral power increases (synchronisation) in the theta, alpha and beta bands during imagery. 

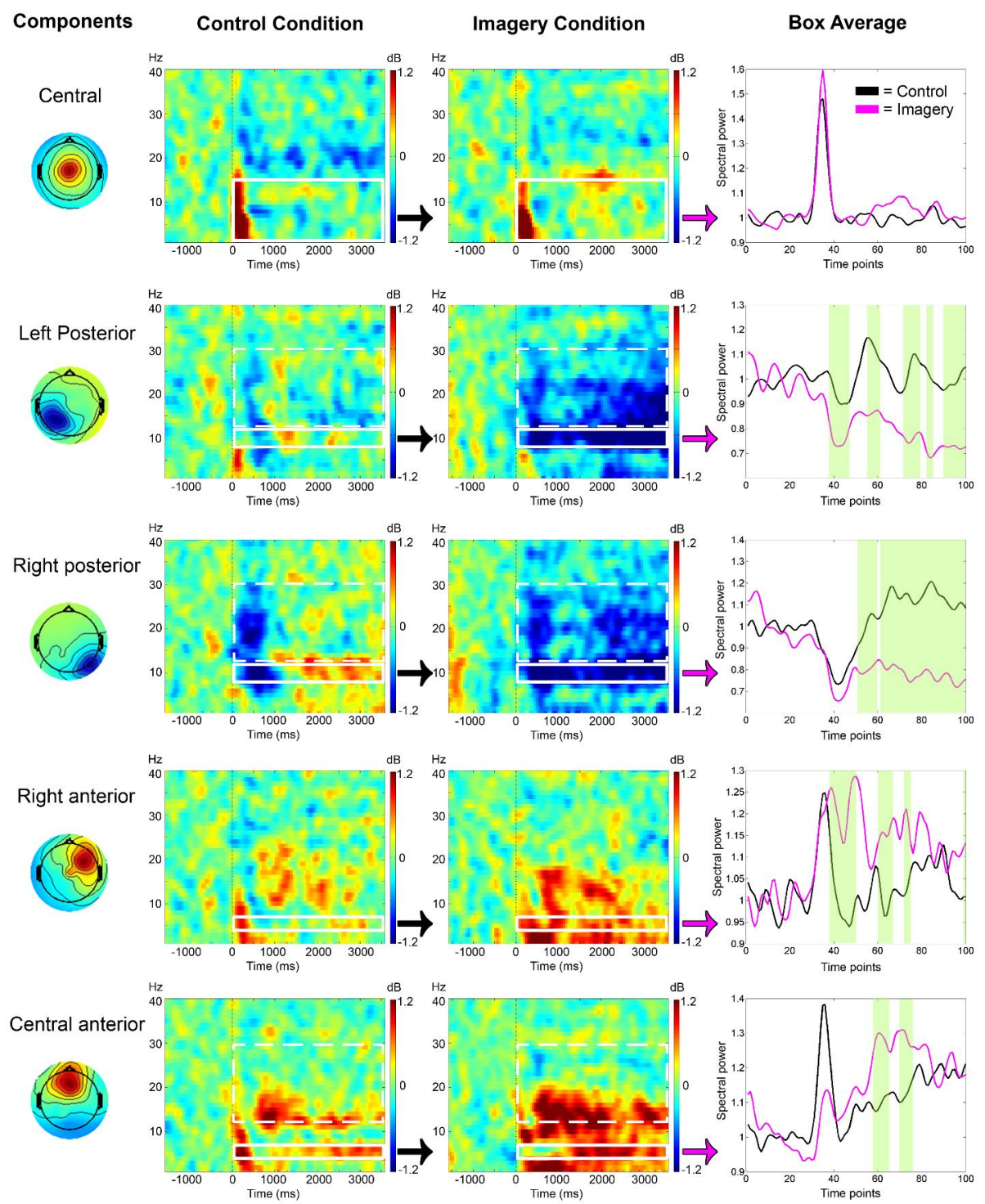

Figure 3.2 Multi-subject averaged event related spectral perturbation (ERSP) plots of five independent components (ICs) Left: Multi-subject averaged ERSP plots and topographical scalp maps in the control and imagery condition. The white continuous boxes indicate the significant time frequency windows, of which the average is displayed on the right. The interrupted white boxes show the significant time frequency windows that are not displayed in more detail. Right: Multi-subject averaged event related spectral power over frequency bands for the control (black) and imagery (purple) conditions. Significant time points $(p<0.05)$ are indicated by green lines. 
The right anterior IC showed significant differences $(\mathrm{p}<0.05)$ between conditions in the theta $[4-7 \mathrm{~Hz}]$ frequency band at several time-points (see Figure 3.2, Box Average, green lines). The central anterior IC showed significant differences $(p<0.05)$ between conditions in the theta [4-7 Hz] (displayed) and beta [12-30 Hz] (similar results, not displayed) frequency bands at several time-points (see Figure 3.2, Box Average, green lines).

\subsubsection{Fast versus slow reaction time trials}

When splitting the trials according to behavioural performance, only one of five components, the central anterior IC, showed a difference between the "fast" and the "slow" condition (Figure 3.3). Paired t-tests revealed significant differences ( $p<0.05)$ in the theta $[4-7 \mathrm{~Hz}]$, alpha $[8-12 \mathrm{~Hz}]$ and beta $[12-30 \mathrm{~Hz}]$ bands at time-points indicated in green, showing that higher spectral power during mental imagery is linked to faster response times on the behavioural task with target pictures. The other four components showed no significant difference between "fast" or "slow" reaction time trials.

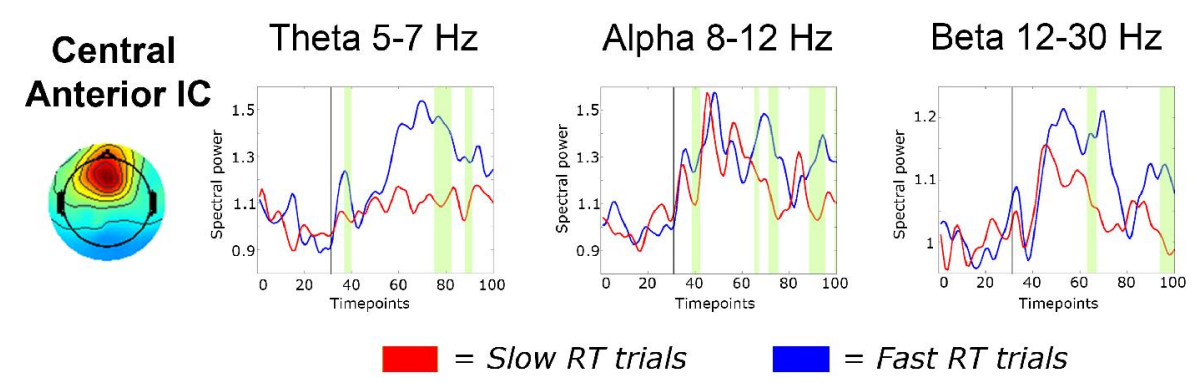

Figure 3.3 EEG results for the comparison of brain responses associated with slow behavioural responses compared to brain responses associated with fast behavioural responses. Left: Topographical map of the central anterior IC. Right: Multi-subject averaged event related spectral power plots of the central anterior IC with significant differences (green) in the theta $(4-7 \mathrm{~Hz})$, alpha $(8-12 \mathrm{~Hz})$ and beta range $(12-30 \mathrm{~Hz})$. 


\subsection{Discussion}

In this study we investigated the spectro-temporal properties of a functional network involved in the imagery of scenes. Our task enforced the spatial analysis of the scene, detailed imagery of the objects in it and maintenance of the complete scene over a period of time. Our results revealed synchronisation of theta and beta rhythms at midline and right frontal sites, contributing to retrieval and integration of visuo-spatial information and de-synchronisation of alpha and beta rhythms in posterior regions, reflecting task complexity and spatial working memory.

\subsubsection{Frontal EEG activity}

Equivalent to the results of the fMRI study in Chapter 2, we found extensive activation patterns in the frontal regions of the brain during EEG measurements. Two different independent components were localized in the anterior part of the brain, the central anterior component and the right anterior component. Both components had early onsets and large sustained power increases (synchronization) in the theta and beta bands. Synchronization of theta power in anterior regions of the brain has been previously associated with working memory. Theta synchronization at right anterior sites is suggested to reflect memory retrieval (Petsche et al., 1992; Bastiaansen et al., 2005; Deiber et al., 2007). Midline frontal theta synchronization has consistently been reported during mental effort, attention and working memory maintenance (Rugg and Venables, 1980; Gevins et al., 1997; Jensen and Tesche, 2002). Compatible with our fMRI results (Chapter 2), several studies support the localization of the midline frontal theta rhythm to anterior cingulate and medial prefrontal cortex (Gevins et al., 1997; Asada et al., 1999; Jensen and Tesche, 2002; Rypma and Prabhakaran, 2009).

As most mental imagery and working memory studies have focussed on the role of theta and alpha oscillations, very little is known about the presence of frontal beta oscillations during mental imagery. Few studies reported beta oscillations during working memory at frontal midline sites (Tallon-Baudry et al., 1998; Onton et al., 2005) or left and right frontal sites (Petsche et al., 1992). The frontal midline beta increase is suggested to relate to integration of information into a rehearsal set (Onton et al., 2005). In the present study, frontal midline beta could reflect the integration of visual and spatial subcomponents into the mental representation of the image. The crucial role of the central anterior component in working memory maintenance and integration of information is reflected by its exclusive influence on task performance. An increase in 
theta and beta oscillations in the central anterior component is accompanied by a decrease in reaction time. These results support the relevance of not only frontal midline theta oscillations, but also of frontal midline beta oscillations in the integration of visuospatial components into a mental scene and its subsequent maintenance in memory. One could conclude that frontal midline theta and beta oscillations enhance mental image construction, resulting in a faster performance on the task.

\subsubsection{Parietal EEG activity}

The posterior regions of the brain at bilateral parietal sites also showed activity during the EEG measurements. Bilateral de-synchronization of alpha and beta oscillations was found in two posterior independent components. Suppression of alpha oscillations has long been associated with an active mental state (Berger, 1930; Slater, 1960). For example, regions that show a localized alpha de-synchronization are considered to be active in information processing or attention (Pfurtscheller et al., 1996). For mental imagery and working memory tasks specifically, posterior alpha de-synchronisation is likely to support memory retrieval and to reflect task complexity (Kosslyn et al., 1997; Neuper and Pfurtscheller, 2001).

We not only found alpha de-synchronization but also a large sustained desynchronization of beta oscillations at posterior sites. Although (posterior) beta desynchronization mostly has been reported within the scope of motor execution and motor imagery, recent studies have also reported posterior beta de-synchronization in the absence of motor responses during memory tasks (Babiloni et al., 2004; Karrasch et al., 2004; Karrasch et al., 2006; Pesonen et al., 2006; Deiber et al., 2007). These studies indicate that beta oscillations with posterior distribution contribute to working memory retrieval and maintenance, most probably reflecting the retrieval and online updating of spatial representational memory. A working memory study, measuring coherence, by Babiloni et al. (2004) suggests that frontal to parietal beta coherence contributes to maintaining spatial information.

Our results showed a significance difference between control and imagery conditions earlier in the left posterior than in the right posterior independent component. This may indicate that left posterior regions were activated earlier, which further supports our previous findings on hemispheric differences between left and right parietal cortex during spatial imagery tasks (Formisano et al., 2002; Sack et al., 2002; Sack et al., 2005). 


\subsubsection{Occipito-temporal EEG activity}

During our EEG measurements we did not find any independent components that could be associated with the involvement of the occipital or ventro-temporal visual regions during mental imagery. This discrepancy between the EEG and fMRI findings (Chapter 2) may result from a lower sensitivity of EEG for these neuronal sources. This is further supported by the fact that independent components reflecting visual processing were found in the analysis of the EEG responses to the target presentation (data not shown), which evoked in fMRI - in the same visual regions - much larger BOLD responses than those observed during imagery (Figure 2.3). Finally, the medial-ventral position of the parahippocampal gyrus makes it highly difficult to measure electrical signals from this region.

\subsubsection{Conclusions}

Our results indicate that memory retrieval during scene imagery is supported by synchronization of theta oscillations in right frontal regions, together with desynchronization in bilateral parietal sites. Theta and beta synchronization in a midline frontal region seems to underlie the integration of information into one visual scene. The general mental imagery process seems to be supported by alpha de-synchronization in posterior sites, which might reflect attention or the inhibition of task non-relevant areas. 
Asada H, Fukuda Y, Tsunoda S, Yamaguchi M, Tonoike M (1999) Frontal midline theta rhythms reflect alternative activation of prefrontal cortex and anterior cingulate cortex in humans. Neurosci Lett 274:29-32.

Babiloni C, Babiloni F, Carducci F, Cincotti F, Vecchio F, Cola B, Rossi S, Miniussi C, Rossini PM (2004) Functional frontoparietal connectivity during short-term memory as revealed by high-resolution EEG coherence analysis. Behav Neurosci 118:687-697.

Barrett J, Ehrlichman H (1982) Bilateral hemispheric alpha activity during visual imagery. Neuropsychologia 20:703-708.

Bastiaansen MCM, Bastiaansen MCM, van der Linden M, Keurs Mt, Dijkstra T, Hagoort P (2005) Theta Responses are Involved in Lexical-Semantic Retrieval during Language Processing. Journal of Cognitive Neuroscience 17:530.

Berger H (1930) Ueber das Elektrenkephalogramm des Menschen. Journal fur Psychologie und Neurologie 40:160.

Bhattacharya J, Petsche H (2005) Drawing on mind's canvas: differences in cortical integration patterns between artists and non-artists. Human Brain Mapping 26:1-14.

Cooper NR, Croft RJ, Dominey SJ, Burgess AP, Gruzelier JH (2003) Paradox lost? Exploring the role of alpha oscillations during externally vs. internally directed attention and the implications for idling and inhibition hypotheses. International Journal of Psychophysiology 47:65-74.

Deiber MP, Missonnier P, Bertrand O, Gold G, Fazio-Costa L, Ibanez V, Giannakopoulos P (2007) Distinction between perceptual and attentional processing in working memory tasks: a study of phase-locked and induced oscillatory brain dynamics. J Cogn Neurosci 19:158172.

Delorme A, Makeig S (2004) EEGLAB: an open source toolbox for analysis of single-trial EEG dynamics including independent component analysis. Journal of Neuroscience Methods 134:9-21.

Farah MJ, Peronnet F, Gonon MA, Giard MH (1988) Electrophysiological evidence for a shared representational medium for visual images and visual percepts. J Exp Psychol Gen 117:248-257.

Formisano E, Linden DE, Di Salle F, Trojano L, Esposito F, Sack AT, Grossi D, Zanella FE, Goebel R (2002) Tracking the mind's image in the brain I: time-resolved fMRI during visuospatial mental imagery. Neuron 35:185-194.

Ganis G, Schendan HE (2008) Visual mental imagery and perception produce opposite adaptation effects on early brain potentials. Neuroimage 42:1714-1727.

Gevins A, Smith ME, McEvoy L, Yu D (1997) High-resolution EEG mapping of cortical activation related to working memory: effects of task difficulty, type of processing, and practice. Cerebral Cortex 7:374-385.

Gill HS, O'Boyle MW, Hathaway J (1998) Cortical distribution of EEG activity for component processes during mental rotation. Cortex 34:707-718.

Holz EM, Glennon M, Prendergast K, Sauseng P (2010) Theta-gamma phase synchronization during memory matching in visual working memory. Neuroimage. 
Jensen O, Tesche CD (2002) Frontal theta activity in humans increases with memory load in a working memory task. European Journal of Neuroscience 15:1395-1399.

Jung TP, Makeig S, Humphries C, Lee TW, McKeown MJ, Iragui V, Sejnowski TJ (2000) Removing electroencephalographic artifacts by blind source separation. Psychophysiology 37:163178.

Kaiser J, Lutzenberger W (2003) Induced gamma-band activity and human brain function. Neuroscientist 9:475-484.

Karrasch M, Laine M, Rapinoja P, Krause CM (2004) Effects of normal aging on event-related desynchronization/synchronization during a memory task in humans. Neurosci Lett 366:18-23.

Karrasch M, Laine M, Rinne JO, Rapinoja P, Sinerva E, Krause CM (2006) Brain oscillatory responses to an auditory-verbal working memory task in mild cognitive impairment and Alzheimer's disease. Int J Psychophysiol 59:168-178.

Klimesch W, Doppelmayr M, Rohm D, Pollhuber D, Stadler W (2000) Simultaneous desynchronization and synchronization of different alpha responses in the human electroencephalograph: a neglected paradox? Neuroscience Letters 284:97-100.

Kosslyn SM, Thompson WL, Alpert NM (1997) Neural systems shared by visual imagery and visual perception: a positron emission tomography study. Neuroimage 6:320-334.

Lehmann D, Henggeler B, Koukkou M, Michel CM (1993) Source localization of brain electric field frequency bands during conscious, spontaneous, visual imagery and abstract thought. Brain Res Cogn Brain Res 1:203-210.

Makeig S, Debener S, Onton J, Delorme A (2004) Mining event-related brain dynamics. Trends in Cognitive Sciences 8:204-210.

Marks DF, Isaac AR (1995) Topographical distribution of EEG activity accompanying visual and motor imagery in vivid and non-vivid imagers. British Journal of Psychology 86:271-282.

Neuper C, Pfurtscheller G (2001) Event-related dynamics of cortical rhythms: frequency-specific features and functional correlates. Int J Psychophysiol 43:41-58.

Nikolaev AR, Anokhin AP (1998) EEG frequency ranges during perception and mental rotation of two- and three-dimensional objects. Neuroscience \& Behavioral Physiology 28:670-677.

Onton J, Delorme A, Makeig S (2005) Frontal midline EEG dynamics during working memory. Neuroimage 27:341-356.

Pegna AJ, Khateb A, Spinelli L, Seeck M, Landis T, Michel CM (1997) Unraveling the cerebral dynamics of mental imagery. Hum Brain Mapp 5:410-421.

Pesonen M, Bjornberg CH, Hamalainen H, Krause CM (2006) Brain oscillatory 1-30 Hz EEG ERD/ERS responses during the different stages of an auditory memory search task. Neurosci Lett 399:45-50.

Petsche H, Kaplan S, von Stein A, Filz O (1997) The possible meaning of the upper and lower alpha frequency ranges for cognitive and creative tasks. International Journal of Psychophysiology 26:77-97. 
Petsche H, Lacroix D, Lindner K, Rappelsberger P, Schmidt-Henrich E (1992) Thinking with images or thinking with language: a pilot EEG probability mapping study. International Journal of Psychophysiology 12:31-39.

Pfurtscheller G, Stancak A, Jr., Neuper C (1996) Event-related synchronization (ERS) in the alpha band--an electrophysiological correlate of cortical idling: a review. International Journal of Psychophysiology 24:39-46.

Rugg MD, Venables PH (1980) EEG correlates of the acquisition of high- and low-imagery words. Neuroscience Letters 16:67-70.

Rypma B, Prabhakaran V (2009) When less is more and when more is more: The mediating roles of capacity and speed in brain-behavior efficiency. Intelligence 37:207-222.

Sack AT, Camprodon JA, Pascual-Leone A, Goebel R (2005) The dynamics of interhemispheric compensatory processes in mental imagery. Science 308:702-704.

Sack AT, Sperling JM, Prvulovic D, Formisano E, Goebel R, Di Salle F, Dierks T, Linden DE (2002) Tracking the mind's image in the brain II: transcranial magnetic stimulation reveals parietal asymmetry in visuospatial imagery. Neuron 35:195-204.

Schupp HT, Lutzenberger W, Birbaumer N, Miltner W, Braun C (1994) Neurophysiological differences between perception and imagery. Brain Res Cogn Brain Res 2:77-86.

Slater KH (1960) Alpha rhythms and mental imagery. Electroencephalography \& Clinical Neurophysiology 12:851.

Tallon-Baudry C, Bertrand O, Peronnet F, Pernier J (1998) Induced gamma-band activity during the delay of a visual short-term memory task in humans. J Neurosci 18:4244-4254. 
Chapter 3

60 


\section{Chapter 4}

\section{Predicting EEG single trials during scene imagery using fMRI and Relevance Vector Machine regression}

\section{Abstract}

Functional MRI (fMRI) and electroencephalography (EEG) have complementary characteristics: fMRI has high spatial resolution, but lagged temporal responses, whilst EEG has high temporal resolution, but faces problems in resolving its neuronal sources. Therefore, the combination of fMRI and EEG has great promise in measuring with high spatial and temporal resolution. In this simultaneous EEG-fMRI study we successfully predict single trial EEG data from fMRI response patterns with Relevance Vector Machine (RVM) regression during the mental imagery of scenes. The prediction of the auditory ERP (cue-related) showed a descriptive pattern of differentiation between regions in auditory cortex for early versus late ERP components. During imagery the significant prediction of ERSP patterns in alpha and beta bands of a centro-frontal component points to the hypothesized coupling between the centro-frontal EEG component and mesial SFG within a network of areas including the bilateral intraparietal sulcus and ventral visual areas.

\section{Based on}

F. De Martino, A.W. de Borst, G. Valente, R. Goebel, and E. Formisano. Predicting EEG single trial responses with simultaneous fMRI and Relevance Vector Machine regression. NeuroImage, Aug 4 (epub ahead of print). 


\subsection{Introduction}

Functional MRI (fMRI) studies on mental imagery over the last decades have given great insight in the network of brain regions involved in imagery processing. Regions in the dorsal processing stream, such as the superior parietal lobe, intraparietal sulcus and precuneus, have been shown to be active during spatial imagery tasks (Mellet et al., 1996; Alivisatos and Petrides, 1997; Knauff et al., 2000; Mellet et al., 2000; Formisano et al., 2002; Sack et al., 2002; Trojano et al., 2004; Sack et al., 2005), whilst category-specific regions in the ventral processing stream, such as the fusiform gyrus or parahippocampal gyrus, have been shown to be active during object imagery tasks (Ishai et al., 2000; O'Craven and Kanwisher, 2000; Ishai et al., 2002). Furthermore, a set of frontal regions such as the mesial superior frontal gyrus and the right medial frontal gyrus, have shown to perform supportive functions during memory retrieval and memory maintenance (Cabeza and Nyberg, 2000). Studies using spectro-temporal defined techniques such as electroencephalography (EEG) and magnetoencephalography (MEG) were able to investigate sub-processes of mental imagery in temporal detail and investigated the role of different frequency bands during imagery. Gamma band oscillations in parietal and occipito-temporal regions seem to be involved during, respectively, spatial and object imagery (Tallon-Baudry et al., 1998; Kaiser and Lutzenberger, 2003; Holz et al., 2010). In frontal regions, theta band oscillations seem to play a role during working memory (Rugg and Venables, 1980; Petsche et al., 1997; Bhattacharya and Petsche, 2005).

These two measurement techniques, fMRI and EEG, have their own specific qualities. Functional MRI can measure with high spatial resolution, but the measured Blood-Oxygenated Level Dependent (BOLD) signal is lagged due to its vascular properties. Electroencephalography on the other hand has great temporal precision, but the spatial source of the signal, as picked up by electrodes on the skull, is highly distorted. Ideally, one would like to make use of a measurement technique that has both high spatial resolution and high temporal resolution. One possible solution is to combine EEG and fMRI measurements in order to achieve this. It has to be taken into consideration though, that fMRI and EEG only partially measure the same neuronal sources. Electroencephalography is most sensitive to only a subpopulation of all neurons: those perpendicular and closest to the skull. Furthermore, the EEG signal seems to reflect (a summation of) excitatory and inhibitory postsynaptic potentials that give rise to the firing or inhibition of neurons (Creutzfeldt et al., 1966; Cooper et al., 1980; Cooper et al., 2003). Functional MRI measures neuronal activity more indirectly, via blood flow, volume and oxygenation in a brain region. The spatial specificity of the signal is thus not directly retraceable to the neurons, as no one to one spatial coupling 
exists between the metabolic changes the BOLD signal arises from and the neuronal activity (Ugurbil et al., 2003; Harel et al., 2006). What neuronal processes exactly lead to an increase in oxygen consumption and thus an increase in BOLD, is not entirely clear. Current studies suggested that both excitatory and inhibitory activity could lead to an increase of BOLD signal, as both influence the regional metabolic energy consumption and blood flow (Ackermann et al., 1984; Raichle, 1998; Lamm et al., 2001). However, a relationship has also been found between inhibitory activity and a negative BOLD signal (Logothetis, 2008). This means that the BOLD signal and the EEG signal could be positively or negatively correlated, or not correlated at all. When combing EEG and fMRI one has to take into account the different physical characteristics of the signals and by design try to optimize the amount of overlap in neuronal sources (Dale and Halgren, 2001; Debener et al., 2006).

The EEG and fMRI measurements can be collected separately or simultaneously. Due to practical limitations or lack of MRI compatible equipment, measurements are often performed separately. However, simultaneous EEG-fMRI measurements have several advantages over separate recordings. The sensory stimulation between modalities is identical, there is no repetition of information leading to learning effects, and the measurement environment and the "state of the brain" (the ongoing fluctuations) are kept constant. One disadvantage of simultaneous stimulation is the degraded EEG signal, due to ballistocardiogram (BCG) and MR-gradient artefacts. These artefacts can be (partly) corrected for, recovering large parts of the signal.

Combined EEG-fMRI measurements can either be used for fMRI-constrained EEG analyses, in order to improve dipole-estimation (Dale and Halgren, 2001), or EEGconstrained fMRI analyses, to find correlates between fMRI data and EEG power modulations (Ritter and Villringer, 2006). The latter is most powerful in order to investigate dynamic cognitive processes. The EEG-constrained fMRI analyses can be performed in several different ways. Most commonly, univariate analyses are applied. Univariate analyses involve extracting single trial event-related potentials (ERPs) and/or the spectral power in certain frequency bands by means of event-related spectral pertubation (ERSP) plots. These responses are then convolved with a hemodynamic response function, in order to create predictors, which are then used in the univariate General Linear Model (GLM) of the fMRI data (Debener et al., 2005; Eichele et al., 2005; Scheeringa et al., 2009). This approach however, does not take the multivariate nature of the data into account. Therefore, a number of multivariate techniques have been developed to analyze EEG-constrained fMRI data multivariately. One of the latest approaches is the use of predictive modelling in EEG-fMRI data analyses. In predictive modelling an algorithm is used to learn a functional relationship between the brain 
response patterns and certain cognitive states (perception of a stimulus, behaviour etc.). After the learning phase, the labelled cognitive states can be predicted from a new dataset (Formisano et al., 2008). Like-wise, this kind of approach can be applied to EEGfMRI analyses, in which the averaged or single trial ERP or ERSP data can be predicted from a learned functional relationship between these EEG data and the fMRI response patterns (De Martino et al., 2010).

In this study we want to investigate the trial-by-trial coupling between single trial EEG data and fMRI response patterns during the mental imagery of scenes by using Relevance Vector Machine (RVM) regression. We hypothesize to find relations between several different cognitive components of our task, as reflected in EEG oscillations and ERPs, and underlying brain response patterns as reflected by fMRI. By showing the ability to predict single trial ERPs and ERSP responses from fMRI response patterns, we hope to enhance understanding of the coupling between EEG rhythms and fMRI responses, specifically during mental imagery. 


\subsection{Methods}

\subsubsection{Participants}

Three healthy right-handed volunteers (3 female, mean age 25 years; range 23-26) participated in the simultaneous EEG-fMRI study. All participants had normal or corrected-to-normal vision. The local ethical committee approved the studies and all participants gave their written informed consent for participation in the study and the MRI safety regulations.

\subsubsection{Task and stimuli}

Twenty-seven digital colour photographs were used as stimuli (screen and picture resolution 1024 by 768 pixels). Three photographs (see example in Figure 4.1, for colour version see Figure 2.1) depicted the interior of a room and comprised several pieces of furniture. These photographs defined the visual imagery content and were solely used during a training phase. Twelve photographs (targets) were fragments of the three original scenes (four per scene), containing a minimum of two partly visible objects. The other twelve photographs (targets) were these same images mirrored across the horizontal axis (see Figure 4.1). The default image was a grey background (50\% black, $50 \%$ white). All photographs contained a centralized white fixation cross and were presented at 5 by $7^{\circ}$ visual angle.

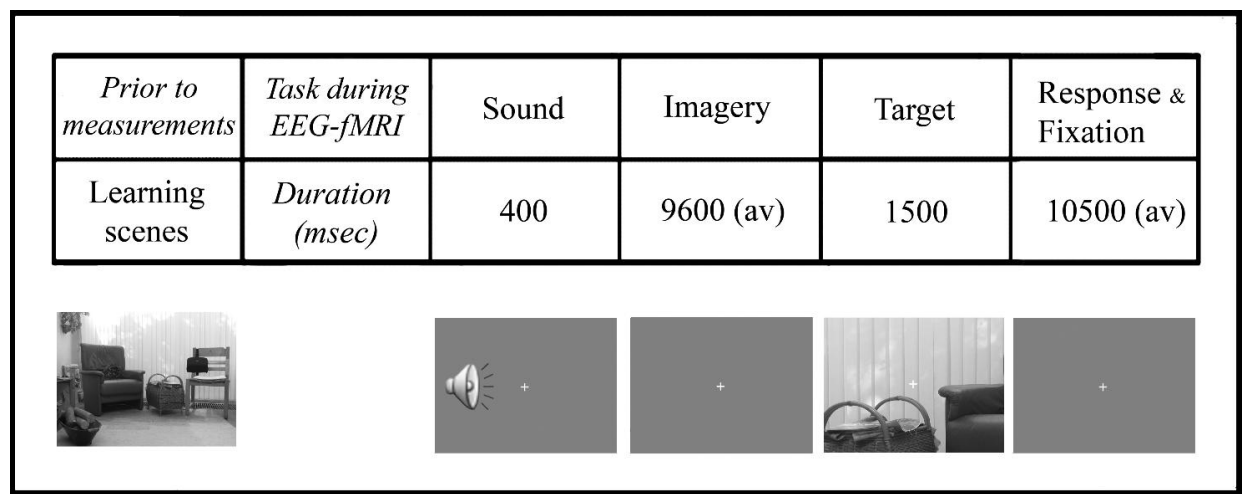

Figure 4.1 Stimulus protocol. Schematic overview of the temporal course of one trial in milliseconds $(\mathrm{ms})$. av $=$ on average. 
The simultaneous EEG-fMRI experiment consisted of 216 ( 3 associations $x 72$ repetitions) trials divided over three runs. A larger number of trials was used, compared to the fMRI experiment (Chapter 2), in order to divide the data in training and testing sets for RVM regression. As depicted in Figure 4.1, each trial of the EEG-fMRI experiment started with an auditory cue (presented in silent gaps during the acquisition, see paragraph 4.2.3). The three auditory stimuli were $400 \mathrm{~ms}$ tones of 800, 1000 and $1200 \mathrm{~Hz}$. During the subsequent interval participants imagined the scene that they previously had learned to associate with the cue. After a variable delay $(7.6,9.6$, or $11.6 \mathrm{~s}$, average $=9.6 \mathrm{~s}$ ) a mirrored or non-mirrored fragment of the scene $(50-50 \%$ distribution) was visually presented as a target for $1.5 \mathrm{~s}$. After each target participants pressed a button with their right middle or index finger to indicate whether the fragment matched the imagined scene, or was mirrored (reversed in half of the participants). All trials ended with a fixation period $(8.5,10.5$, or $12.5 \mathrm{~s}$, average $=10.5 \mathrm{~s})$ during which the participants performed no task. Timing was adapted from the orignal fMRI design (Chapter 2) to fit the silent gap paradigm and to have an adequate number of time points for subsequent analysis steps. The interval lengths were randomized over trials and the occurrence of each period length was equally distributed over the three types of trials (scene one, two and three) and type of target picture. The trials (e.g. the order of the tones and target pictures) were randomized.

Prior to EEG-fMRI measurements, participants were trained on imagining three scenes, associated with three auditory cues. The training session consisted of 24 randomized trials of simultaneous presentation of the auditory stimulus and the corresponding visual scene (4.4 s), followed by a fixation period ( $8 \mathrm{~s}$ ) during which the participant imagined the scene. After training, participants received task instructions and were asked to vividly visualize the correct scene after each cue in the main experiment, while fixating at the fixation cross.

\subsubsection{Data acquisition}

\section{Electroencephalography}

Electroencephalography was recorded using a 64-channel high-input impedance amplifier system specifically designed to operate in the MRI environment (Brainproducts, Munich, Germany). The setup further consisted of two 32-channel MR plus amplifiers powered by a rechargeable power unit. The amplifiers were placed directly behind the scanner bore inside the MR room, which allowed the use of short 
wires with a total length of about $1.2 \mathrm{~m}$ from recording electrodes to amplifier. Sintered $\mathrm{Ag} / \mathrm{AgCl}$ ring electrodes with built-in $5 \mathrm{k} \Omega$ resistors were used. Data were recorded from 62 equidistant electrodes mounted in a cap system (Easycap, Falk Minow Services, Herrsching, Germany). Continuous data were also recorded from one electrode placed below the left eye, to monitor eye blinks, and another electrode placed at the lower back for electrocardiogram (ECG) recordings. Electrode impedances were maintained below $10 \mathrm{k} \Omega$ before recordings. All 64 channel data were referenced to the vertex. The data were recorded with a pass-band of $0.016-250 \mathrm{~Hz}$ and digitized with 5000 samples $/ \mathrm{s}$ at 16bit resolution, resulting in a dynamic range of $16.38 \mathrm{mV}$. The amplified signal was transmitted via fiber-optic cables to a recording PC placed outside the MR room.

\section{Functional MRI}

A 3T Siemens MR (head) scanner (MAGNETOM Allegra, Siemens Medical Systems, Erlangen, Germany) was used for imaging. Three functional scans were acquired with a Gradient Echo Echo Planar Imaging sequence with a Repetition Time (TR) of 2000 ms and a Time of Acquisition (TA) of 1400ms. For each functional run 792 volumes were acquired comprising 22 slices (matrix $=64 \times 64, \mathrm{FOV}=224 \times 224$, voxel dimensions $=3.5 \mathrm{x}$ $3.5 \times 4 \mathrm{~mm} 3$, gap $=0.72 \mathrm{~mm}$, interslice time $=90 \mathrm{~ms})$. Afterwards, high-resolution T1weighted structural images of the whole brain were acquired (ADNI MPRAGE 192 slices, matrix $=256 \times 256$, voxel dimensions $=1 \times 1 \times 1 \mathrm{~mm} 3, \mathrm{TR}=2250 \mathrm{~ms}$ ).

\subsubsection{Data analyses}

The use of predictive models requires both data sets (i.e. the pre-processed fMRI time series and the EEG-based predictors) to be divided into training and testing sets. The training set is used to learn the coupling between the fMRI and the EEG signal at a trialby-trial level using Relevance Vector Machines (RVM). Afterwards, a map depicting the contribution of each voxel to the learned coupling is obtained. The test data are used to assess the validity of the learned coupling.

\section{Electroencephalography pre-processing}

An open source Matlab toolbox, EEGLAB (Delorme and Makeig, 2004), was used for 
EEG data processing. Functional MRI gradient and ballistocardiogram (BCG) artefacts were removed from the signal using the EEGLAB plug-in FMRIB 1.21 (Niazy et al., 2005), as developed by the Centre for the Functional MRI of the Brain (Oxford, UK). The three runs were concatenated, ground and reference channels were removed and the data were re-referenced to an average reference. For each subject temporal Independent Component Analysis (ICA) was used to extract 62 components (64 channels, minus ground and reference). The Independent Components (ICs) were visually reviewed in order to discard components reflecting residual BCG or gradient artefacts (Debener et al., 2007). Afterwards, channels with a high noise level were removed and the continuous data were epoched around the auditory cue (-1000 to $7500 \mathrm{~ms}$ ) and the visual target (-1000 to $1000 \mathrm{~ms}$ ). Epochs exhibiting clear artefacts (eye blinks or muscle artefacts) were removed prior to subsequent processing steps.

\section{Electroencephalography ICA}

After artefact removal, new ICAs were performed on the two epoched datasets to find components of interest associated with the task. The unsupervised nature of ICA does not bias the subsequent use of predictive models as no labels are used in the extraction of the components. In this study, relevant independent components (ICs) were manually selected based on the inspection of topography, event related potentials (ERP), event related spectral power (ERSP) plots, across subject consistency and correspondence to the results obtained in a separate EEG study conducted outside the MR scanner (Chapter 3).

\section{Electroencephalography ERSP analyses}

A time frequency decomposition (Morlet wavelets; window size $=1119 \mathrm{~ms}$ ) was applied on the time courses of the ICs in order to extract the power modulations in the band [2 $28 \mathrm{~Hz}$. Event related spectral power plots were calculated for all components. After selection of the cognitively relevant components, the modulation of power in different time-frequency windows (characterized by a continuous response over extended time windows) was estimated for every trial of the selected ICs. For the estimation of the single trial power modulations the mean in a baseline period [-200 to $0 \mathrm{~ms}$ ] was removed for each frequency and the time-frequency window was averaged over frequency. This estimation procedure resulted in a signal with power values for each time point within 
the selected time-frequency window, which was subsequently convolved with the hemodynamic response function. Frequency and time windows were chosen based on the average ERSPs of the selected ICs. All single trial measures were estimated using customized software implemented in Matlab (The MathWorks, Inc.).

\section{Electroencephalography ERP analyses}

Furthermore, ERPs were calculated for all ICs by averaging the component time-courses over trials. After component selection, ERPs of the stimulus-locked events, i.e. the ICs representing the auditory cue and target picture processing, were used for further analyses. For the different characteristic components of the ERP (e.g. auditory N1, visual P1, P2) peak and latency values were estimated for every trial. In order to estimate the single trial ERPs, the mean in a baseline period [-200 to $0 \mathrm{~ms}$ ] was removed from every single trial in the selected IC time course and the maximum (or minimum) value and its latency were extracted from temporal windows $(50 \mathrm{~ms})$ selected around the peaks of the average ERP response (e.g. auditory N1, visual P1). This procedure resulted in one single point (time, amplitude) per single trial as a representative of the selected peak response.

Single trial information coming from the ERP and the ERSP was subsequently convolved with a standard hemodynamic response function (HRF, Friston et al., 1998) and re-sampled to the fMRI sampling rate $(0.5 \mathrm{~Hz}$, see below) to obtain ERP-based and ERSP-based predictors. Prior to the regression procedure all predictors were $\mathrm{z}$ normalized.

\section{Functional MRI pre-processing}

All functional MRI data were analysed using fMRI analysis and visualisation software BrainVoyager QX (Brain Innovation B.V., Maastricht, The Netherlands). Functional data were 3D motion corrected (trilinear interpolation), corrected for slice scan time differences and temporally filtered (high pass: 5 cycles/run cut-off). No spatial filtering was applied. The anatomical data were corrected for intensity inhomogeneity (Goebel et al., 2006) and transformed into Talairach space (Talairach and Tournoux, 1988). The functional data were then aligned with the anatomical data and transformed into the same space, to create $4 \mathrm{D}$ volume time-courses (VTCs). The anatomical data were used for surface reconstruction of both hemispheres in all subjects (description of cortex segmentation in Goebel et al., 2006). Cortex-based alignment was performed in order to 
optimize display of group-results on the cortex.

\section{Functional MRI data analyses}

Statistical modelling of the time-courses was based on a general linear model with a design matrix implementing a deconvolution analysis. An estimate of the hemodynamic response at each TR during a $22 \mathrm{~s}$ window after trial onset was obtained using a separate stick predictor. The group map was based on the concatenation of single-subject deconvolution matrices (fixed effect GLM; $\mathrm{p}$ (Bonf corr) <0.05). A map of BOLD response latency (Figure 4.2) was obtained by contrasting the first five stick predictors (sound and imagery) with the last eight stick predictors (target) and colour coding significantly active voxels according to the earliest stick predictor for which the beta weights reached significance (i.e. a green colour indicates a significant beta weight for the stick predictor corresponding to the first TR after sound onset, a blue colour to second TR, etc.). The results of this separate analysis were only used for data inspection and visualization purposes.

\section{Relevance Vector Machine regression}

The data sets of the three functional runs of each subject were used for the multivariate regression analysis using Relevance Vector Machines (RVM). Training was performed using the data of two runs (e.g. run 1 and 2) and predictions were evaluated on the data from the left out run (i.e. leave-one-run-out strategy). Pre-selection of voxels was performed to reduce the number of irrelevant features (voxels). Voxel pre-selection was based on conventional univariate analysis (GLM with predictors accounting for the auditory cue, delay period and visual target) performed only on the training data (i.e. separately for each cross-validation step). The most significant $(\mathrm{N}=5000)$ voxels according to an F-statistic were used for the subsequent RVM training procedure. The RVM regression analyses were performed for each EEG predictor separately. To obtain group maps after training of the RVM for the different predictors of one IC, single subject maps were sampled on the cortex, cortically realigned, thresholded (to select the $40 \%$ most relevant vertices for the prediction) and binarized. A vertex in the group map is colour coded if present in at least two of the three single subject maps (Figure 4.5). Cross-correlations between predicted and actual responses were calculated for each predictor. In order to validate whether single trial modulations contributed to the 
predictions or whether good predictions were caused by a general hemodynamic response effect, i.e. high correspondence between the BOLD response and the HRF convolved EEG response (largely matching the experimental protocol), several different approaches were used. Details on the RVM regression procedure and the evaluation of single trial modulations can be found in the Supplementary Materials (paragraph 4.5). 


\subsection{Results}

\subsubsection{General fMRI results}

Figure 4.2 shows the latency mapping of BOLD activation during mental imagery of scenes. In accordance with results from a separate non-simultaneous fMRI study (de Borst et al., submitted; Chapter 2) a similar spatio-temporal pattern can be descried. An early activation of auditory cortex (AC), superior temporal gyrus (STG), right superior temporal sulcus (STS) and right insula (INS) after the presentation of the auditory cue (green and blue colour) can be observed. Afterwards, a large fronto-parietal network including superior parietal lobule (SPL) and intra parietal sulcus (IPS) together with parts of the mesial superior frontal gyrus (SFG) (extending from the supplementary motor area to the anterior cingulate cortex), pre-motor cortex (PreM) and INS were activated during the imagery period (yellow and light orange color). With the presentation of the target (included in Figure 4.2, not present in Chapter 2) visual regions (parts of the visual cortex and the parahippocampal gyrus) and motor regions (SFG, PreM and left motor cortex) became active (dark orange).

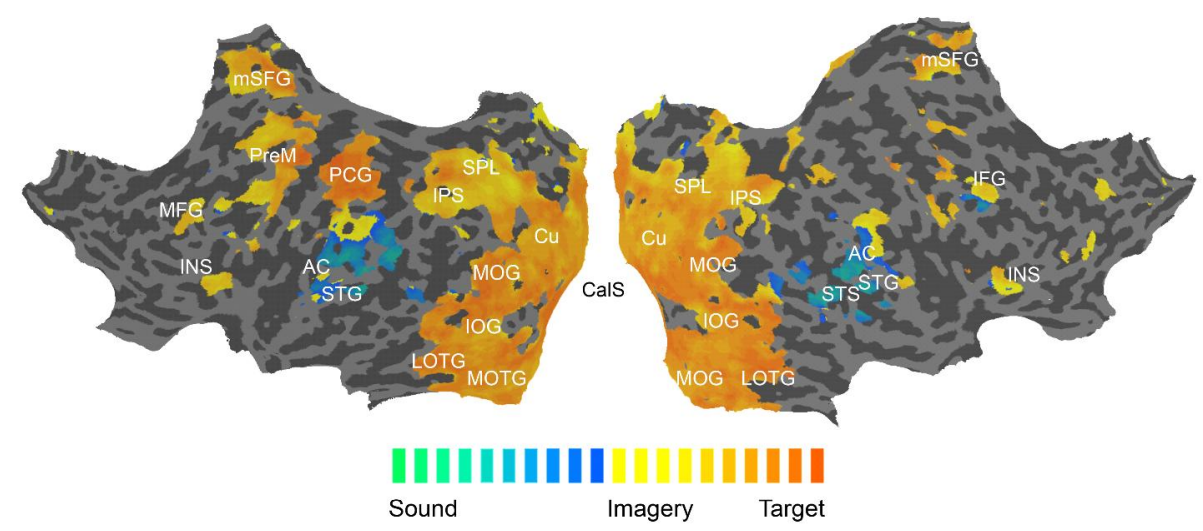

Figure 4.2 Multisubject (deconvolution) map of brain activation during the mental imagery of scenes, superimposed on a flattened representation of the cortical sheet. The voxels are colour coded according to their first significant beta value, representing the latency of BOLD activation during the auditory cue, mental imagery and target processing. Left to right: MFG = middle frontal gyrus, INS = insula, $\mathrm{mSFG}=$ mesial superior frontal gyrus, PreM = premotor cortex, AC = auditory cortex, STG = superior temporal gyrus, PCG = postcentral gyrus, LOTG = lateral occipital temporal gyrus, IPS = intraparietal sulcus, SPL = superior parietal lobule, IOG = inferior occipital gyrus, MOTG = medial occipital temporal gyrus, MOG = middle occipital gyrus, $\mathrm{Cu}=\mathrm{Cuneus}, \mathrm{CalS}=$ calcarine sulcus, IFG = inferior frontal gyrus. 


\subsubsection{General EEG results}

Event-related potentials or spectral power changes of three independent components were used for the EEG analyses and subsequent RVM regression. The selection of these components was based on the findings of a separate non-simultaneous EEG study with the same mental imagery paradigm (Chapter 3), between-subject consistency and features of the ERP, ERSP and topography plots. These components reflected the three main elements of the task: auditory cue processing, mental imagery of the scene and target processing. Because cue and target processing are both stimulus-locked EEG phenomona, ERPs were extracted after epoching to either the cue or target. Since mental imagery is not a stimulus-locked process, an ERSP was extracted for this component. Figure 4.3 shows the group topography, the ERP and the ERSP of two components extracted after epoching to the auditory cue (AI) and the ERP of one component extracted after epoching to the target (VT). Figure 4.3a shows a central component related to the processing of the auditory cue. The central distribution is common in a bilateral temporal activation. The ERP shows an early negative peak around $100 \mathrm{~ms}$ (N1), followed by a positive peak around $200 \mathrm{~ms}$ (P2) and sustained negative activity $(\mathrm{SN})$. Figure $4.3 \mathrm{~b}$ shows a central anterior component related to integration of information during mental imagery (as shown in a separate EEG study, Chapter 3).
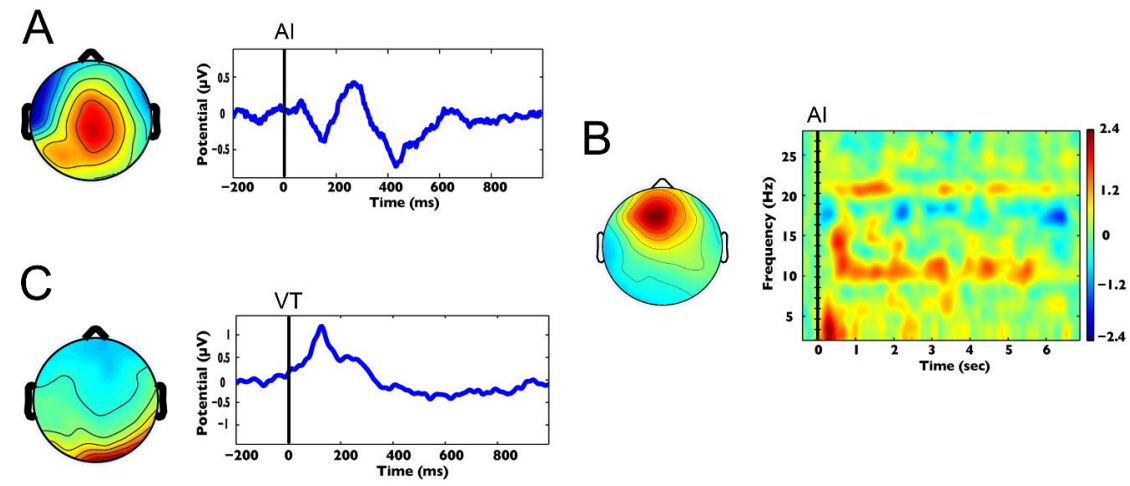

Figure 4.3 Topography, ERP and ERSP plots of three EEG independent components. A: Topography and ERP plot (epoched at Auditory Instruction [AI]) of central component. B: Topography and ERSP plot (epoched at AI) of central frontal component. C: Topography and ERP plot (epoched at Visual Target [VT]).

The ERSP data show synchronization in alpha (8-12 Hz) and beta (19-23 Hz) frequency bands. Figure $4.3 \mathrm{c}$ shows a central posterior component related to the visual processing of target information. The ERP shows an initial positive peak around $100 \mathrm{~ms}$ (P1). 
Considering consistency, physiological plausibility and functional interest, the described properties of these three ICs were used in the subsequent fMRI-based predictions.

\subsubsection{Auditory ERP predictions}

In order to understand the relation between fMRI and EEG responses following the auditory instruction, RVM regression of $\mathrm{fMRI}$ data was used to predict the prominent ERP peaks of the early auditory IC (Figure 4.4a). The predictors (after HRF convolution and resampling to the fMRI sampling frequency) obtained for the three separate peaks (N1, P2 and SN, see Figure 4.4a, right) were used for RVM training in three separate analyses. Figure $4.4 \mathrm{~b}$ shows the "group" map, resulting from the training of the RVM on the three different ERP based predictors (N1, P2 and SN). The map represents the union of the maps obtained for the three different peaks in the single subjects (see Methods RVM). The map shows regions in the auditory cortex (mostly right hemisphere), bilateral intraparietal sulcus (IPS), mesial superior frontal gyrus (SFG), inferior occipital gyrus (IOG) and medial occipito-temporal gyrus (OTG) to be relevant for predictions of the auditory components.

The predictive patterns for each of the three peaks in the auditory cortex specifically, are represented in Figure 4.4c. The colour coding in these maps is according to the best $60 \%$ (red) and $40 \%$ (orange) of the voxels, thresholded in the single subject maps. The patterns relating to the N1 and P1 include a region in the lateral aspect of the Heschl's Gyrus and a more posterior region on the planum temporale, while the pattern relating to the $\mathrm{SN}$ only shows the posterior region. Cross-correlations between predicted and actual responses were computed after applying a Fisher $r$ to $\mathrm{z}$ transform to the single subject accuracies and then transforming the mean score back to $r$ (red dot, Figure $4.4 \mathrm{~d})$. They were significant in all cases $\left(\mathrm{N} 1, \mathrm{cc}=0.51, \mathrm{p}=5.4 \cdot 10^{-32} ; \mathrm{P} 2, \mathrm{cc}=0.58, \mathrm{p}=4.1 \cdot 10^{-}\right.$ 42; $\mathrm{SN}, \mathrm{cc}=0.63, \mathrm{p}=1.9 \cdot 10^{-51}$; $\mathrm{p}$-values computed using the number of points in each predicted run $[n=450])$. See paragraph 4.4 Supplementary Materials for the evaluation of single trial modulations. 


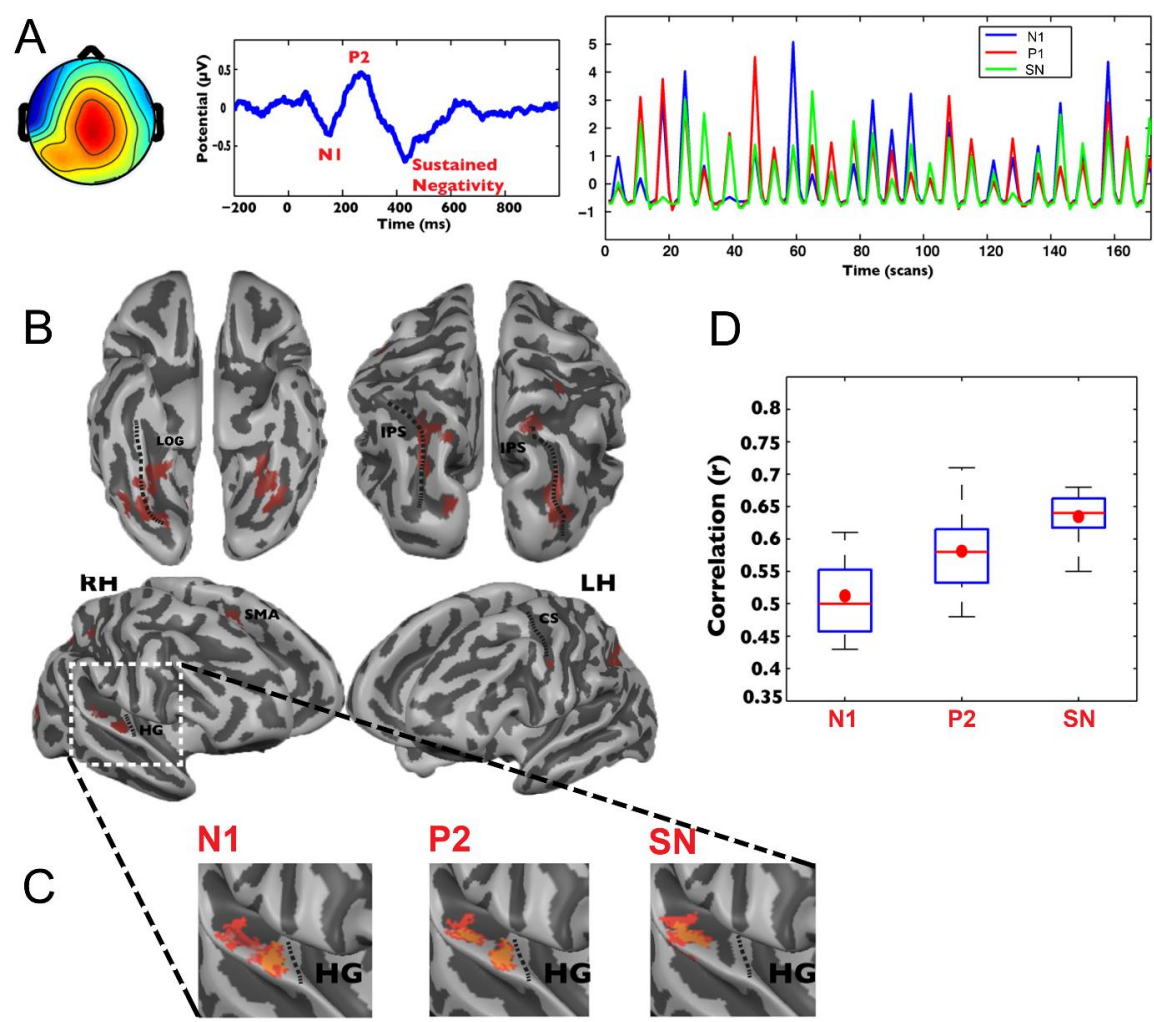

Figure 4.4 Results from the auditory ERP predictions. A. Representation of the group topography, ERP components N1, P2 and SN (SN) and the three corresponding predictors. B. Map of the results of RVM training on the three predictors over three subjects represented on a ventral, posterior and lateral view of a cortex-based aligned group representation of the cortex. C. Zoomed-in portion of Heschl's gyrus, showing predictive patterns for each of the three ERP components separately. Red colour indicates the best $60 \%$ of the predictive voxels, orange the best $40 \%$. D. Box plots of the cross correlations between actual and predicted response for each ERP component $($ red dot $=$ mean score, red line $=$ median $)$.

\subsubsection{Central anterior ERSP predictions}

Following a similar procedure as the auditory predictions, a RVM regression analysis was performed on two time-frequency (TF) windows within the alpha [9-12 Hz] and beta $[19-23 \mathrm{~Hz}]$ frequency bands of the central anterior component. The results of this analysis are shown in Figure 4.5. The topography and ERSP plot of the central anterior component, that in Chapter 3 was found to be essential for the imagery of scenes, is shown in Figure 4.5a. 

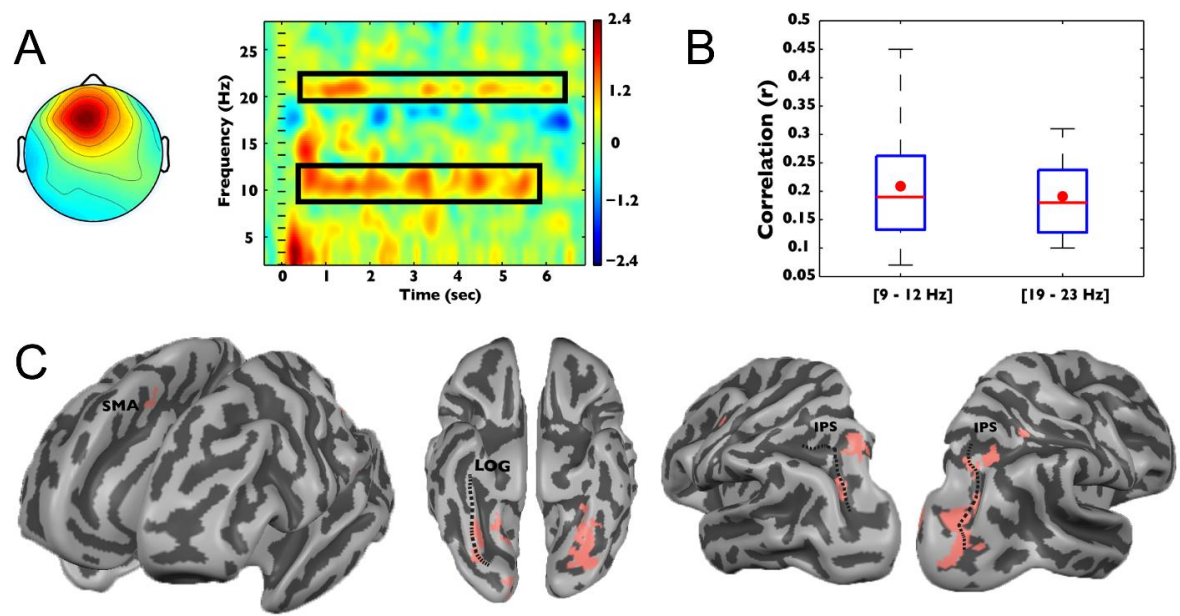

Figure 4.5 Results from the imagery ERSP predictions. A. Group topography and ERSP plot of the central anterior component. The black boxes indicate the selected time-frequency windows within the alpha and beta bands [9-12 $\mathrm{Hz} ; 19-23 \mathrm{~Hz}$. B. Box plots of the cross correlations between actual and predicted responses for the two timefrequency windows (red dot $=$ mean score, red line = median). C. Map of the results of RVM training on the two predictors over three subjects, represented on an anterior, ventral and posterior view of a cortex-based aligned group representation of the cortex.

Figure $4.5 \mathrm{~b}$ shows the group map after cortex based alignment obtained from the training of the RVM in each subject (thresholding as described in Methods - RVM). The map highlights areas in bilateral IPS, mesial SFG, IOG and medial OTG to be relevant for predictions of alpha and beta power modulations in the central anterior component. Figure $4.5 \mathrm{c}$ shows that the group accuracy values (computed as in figure $4.4 \mathrm{~d}$ ) for the prediction of the two selected time-frequency windows in the central anterior component are significant, but lower compared to the ERP results of the auditory component $\left([9-12 \mathrm{~Hz}] \mathrm{cc}=0.21, \mathrm{p}=3.7 \cdot 10^{-6} ;[19-23 \mathrm{~Hz}] \mathrm{cc}=0.19, \mathrm{p}=2.1 \cdot 10^{-5}\right)$. Significance for the predictions was computed based on the mean $\mathrm{z}$ value and the minimum time samples in each run $(n=450)$.

\subsubsection{Central posterior ERP predictions}

Predictions using RVM regression were also applied to the P1 peak of a visual ERP related to the target presentation. Here, the EEG data were epoched with respect to the visual target (-1000 till $1000 \mathrm{~ms}$ ). The IC shows activation at occipital electrodes in the topography, which is reflected in the P1 peak of the ERP (Figure 4.6a). Figure 4.6b shows 
the group map after cortex based alignment obtained from the training of the RVM in each subject on the prediction of the P1 peak (thresholding as described in Methods RVM). This map highlights regions in the calcarine sulcus (CalS) and lateral occipital gyrus (LOG). Group accuracy is shown in Figure 4.6c ( $\mathrm{cc}=0.75 ; \mathrm{p}=4.9 \cdot 10^{-86}$, computed based on the mean $\mathrm{z}$ value and the minimum points in each run [ $\mathrm{n}=450]$ ). The prediction accuracies on this ERP component are highly significant. However, these accuracies are largely determined by the dominating effect of the overall hemodynamic response to the visual stimulus (see 4.3.2 Methodological considerations and 4.4 Supplementary Materials).

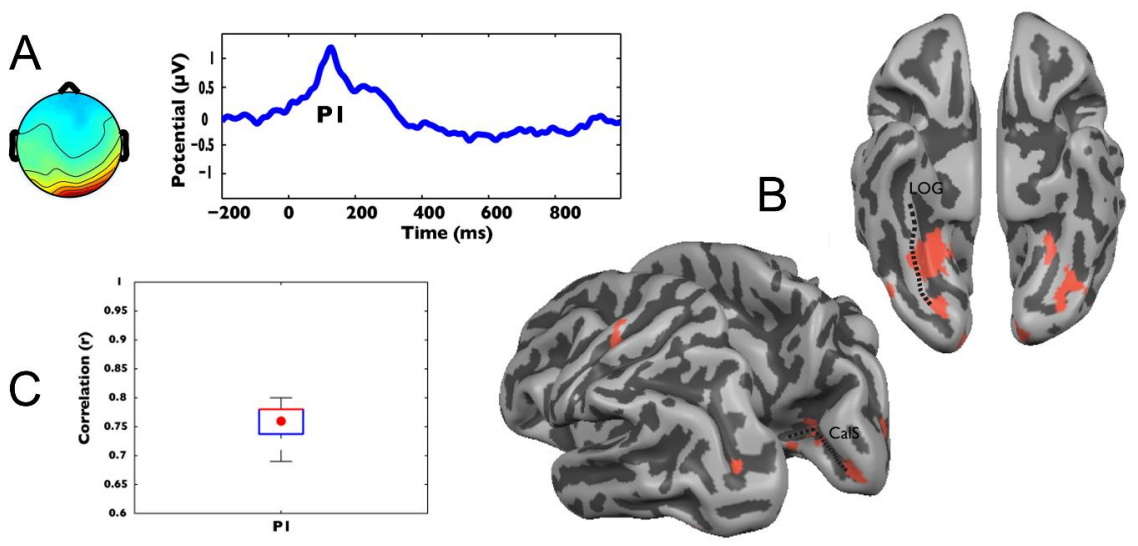

Figure 4.6 Results from the visual ERP prediction. A. Group topography and the ERP component P1. B. The map of the results of the RVM training on the P1 predictor over three subjects represented on a posterior and ventral view of a cortex-based aligned group representation of the cortex. C. Box plot of the cross correlations between actual and predicted response for the ERP component (red dot $=$ mean score, red line = median). 


\subsection{Discussion}

In this study, we have introduced the use of multivariate regression and predictive modelling as an analysis-tool of trial-by-trial coupling in simultaneous EEG-fMRI measurements. We aimed to predict event related modulations of the EEG from multivariate fMRI patterns during a complex scene imagery task. The nature of the task enabled us to apply these analysis-tools to several different cognitive processes: auditory perception (cue), visual imagery (task) and visual perception (target). Beyond a simple correlation between the two signals, we have shown that it is possible to predict both stimulus-locked (ERP) and oscillatory (ERSP) EEG activity from multivariate patterns as measured by fMRI during different aspects of the task. The results indicate a possible latency difference between two involved regions in auditory cortex: a region close to Heschl's gyrus seems to be more predictive for early auditory ERP peaks, and not for the later $\mathrm{SN}$, compared to a more posterior region. During imagery, the results confirm a suggested coupling between mesial SFG and a central anterior EEG component, within a network of several other regions, such as IPS and ventral temporal cortex.

\subsubsection{RVM predictions}

\section{Functional MRI and EEG results}

Consistent with the results of Chapter 2, analysis of fMRI data collected during the mental imagery task showed that a large network of brain regions was activated, including auditory regions (due to auditory cue), frontal regions such as mesial SFG and premotor cortex, parietal cortex and ventral temporal regions (imagery). After presenting the visual target many of these same regions were re-activated together with extensive parts of the visual cortex.

As for the simultaneously-collected EEG data, we compared the results to those of a previous study with the same paradigm, where the EEG was acquired outside the scanner (see Chapter 3). We selected one component related to the auditory processing of the cue, one component related to working memory integration during imagery, and one component related to visual processing of the target. Since auditory and visual processing was stimulus-locked, ERPs were extracted for these components and ERP peaks (auditory N1, P2 and SN; visual P1) were used in subsequent RVM analyses. The visual imagery was not stimulus-locked and therefore an ERSP plot was extracted and relevant time-frequency windows were selected for RVM regression. 


\section{Auditory ERP predictions}

Our results showed that RVM regression is able to capture the modulations of the ERP peaks associated with the processing of the auditory cue (N1, P2 and SN). Maps obtained from the RVM-training (Figure 4.4) show that a broad network comprising the auditory cortex, right superior temporal gyrus, intra-parietal sulcus and mesial superior frontal gyrus, was relevant for the prediction of the auditory ERP peaks. Within auditory cortex, the maps obtained from the prediction of the three peaks of the auditory ERPs separately, suggest a temporal difference between regions of the auditory cortex closer to the Heschl's gyrus and more posterior regions. The regions closer to Heschl's gyrus seem to be more relevant for prediction of the early auditory ERP peaks N1 and P2, and not for the SN. This seems physiologically plausible, as early perceptual processing, reflected in N1 and P2, involves primary auditory cortex in Heschl's gyrus (Formisano et al., 1998), while more semantic processing of the cue as reflected in the later sustained negativity may involve more posterior regions (Friederici et al., 1998). Considering the small number of subjects and the empirical thresholding procedure employed, however, these maps are to be considered as a qualitative description of the informative patterns and the statistical validity of the observed difference needs to be addressed in further studies with a larger number of subjects.

\section{Mental imagery ERSP predictions}

The EEG results highlighted the contribution of a centro-frontal EEG component to the mental imagery process (Figure 4.3). In a previous EEG study with the same paradigm (de Borst et al.; Chapter 3) performed outside the MR scanner, theta and beta synchronization of a similar centro-frontal component during mental imagery was shown to be predictive of later task performance. In this study we were able to predict ERSP modulations in the alpha and beta bands of the centro-frontal component. The maps obtained from the training of the RVM (Figure 4.5) point towards a hypothesized coupling between the centro-frontal EEG component and the mesial SFG (Chapter 3) within a network of areas including the bilateral intraparietal sulcus and ventral visual areas.

The prediction of such ERSP modulations led to lower (but still significant) accuracy values compared to the ERP-based predictions. This was probably due to the fact that the predictors for the imagery period were obtained from a temporal window encompassing almost the whole trial length. Also, brain activity due to mental imagery 
is less stimulus-locked than perceptual processing of sounds or images. These ERSP predictors were therefore less affected by the issue of a high degree of correlation with the experimental protocol compared to the auditory and visual predictors.

\section{Visual ERP predictions}

To further confirm that our method can produce physiologically plausible results we also predicted the ERP response associated with the presentation of the visual target (Figure 4.6). The map obtained from the training of the RVM showed relevance of early visual regions and higher order visual processing regions in ventral temporal cortex. While highly significant, these results, as well as the auditory ERP predictions, are contaminated by the overall correspondence between the convolved predictors, the fMRI signal and the experimental protocol. In order to evaluate whether specific EEG information contributes to the predictions, a number of analyses were performed (4.5 Supplementary Materials). These results will be briefly discussed below.

\subsubsection{Methodological considerations}

The RVM-based predictions led to high correlation values between predicted and actual test data. However, because of the filtering effects of the hemodynamic response, such high values are largely determined by the overall variations of the fMRI signal with the auditory and visual stimulus (the on-off type of response to the sound/image), which makes it difficult evaluating the actual contribution of EEG modulations.

In order to evaluate single-trial EEG contributions to the predictions three analyses were performed on the auditory ERP predictions (4.5 Supplementary Materials). The results of these analyses showed that the overall prediction accuracies between EEG informed and non-informed (according to stimulus protocol) predictors were similar (Table 1). This seems to indicate that the high values of correlation between predicted and actual response simply reflected a good prediction of the hemodynamic response to the sound presentation but not necessarily of the single-trial EEG fluctuations. When evaluating the contribution of EEG modulations (orthogonalized to the protocol) and the experimental protocol to the predicted time-courses with a posthoc GLM analysis, the results showed a significant contribution of the EEG information to the prediction of each peak specifically (Figure 4.7, Evaluation analysis 1). When the fMRI time-series used for training are orthogonalized to the stimulus protocol (the 
auditory response), and thus circumvent the above described problem, the prediction accuracies are much lower, but still significant for the predictions of P2 and the SN (Table 2, Evaluation analysis 2). The information in the maps (Figure 4.8, Evaluation analysis 2) is largely maintained, showing a benefit of including EEG information compared to no EEG information. In a final analysis the trial-to-trial correlations between the predicted and actual EEG responses for EEG-informed predictors based on the non-orthogonalized data and the orthogonalized data and a predictor based on only protocol information were calculated (Table 3, Evaluation analysis 3). These data showed an advantage of the EEG informed training over the non-EEG informed training, where the EEG informed training had higher correlation values. The EEG informed training based on the orthogonalized data analyzed on a trial-by-trial basis showed higher correlation values but lower significance (Table 3), compared to the whole time-course based analyses (Table 2). Overall there is much variance in significance of the trial-bytrial correlations, and over the runs/subjects only the P2 and SN correlations with the EEG informed non-orthogonalized data are significant.

Overall, these three different analyses of the auditory event-related responses indicate that considering the information of single-trial modulations in training data provides a small but significant improvement in predicting modulations of corresponding EEG responses in test data. The variability of the results at single run/subject level indicates that - although promising - this approach needs further improvement and optimization to allow an accurate trial-by-trial prediction.

\subsubsection{Conclusions}

This study showed that multi-variate regression is a valuable tool for investigating the relationship between fMRI and EEG single trial modulations. We have shown that synchronization in alpha and beta bands in an anterior frontal component can be predicted from a multi-variate brain pattern including regions in mesial SFG, IPS and ventral temporal cortex. Furthermore our results indicated a differential brain pattern for the prediction of the early auditory ERP peaks compared to the SN. Additional methodological analyses showed that, although effects are small, including single trial modulations in the RVM training does improve the prediction of EEG responses. The use of prediction based modelling approaches as RVM thus allows evaluating the real predictive power of one modality (fMRI) on the other (EEG). 


\subsection{Supplementary Materials}

In this chapter the details on RVM regression and the evaluations of the used methods are described.

\subsubsection{Detailed RVM methodology}

\section{Relevance Vector Machine regression background}

Here, the concepts of RVM that are relevant to our application on EEG-fMRI data are briefly reviewed. For a complete description of RVM see Tipping (2001) and Formisano et al. (2008). "Let us consider a data set D consisting of an fMRI time series, represented by an $N \times V$ matrix $\mathbf{X}$ ( $N$ being the number of volumes and $V$ the number of voxels), and an EEG based predictor, represented by the $N$ dimensional vector $\mathbf{p}$. Such a dataset can be described as a collection of $N$ pairs $\left(\mathbf{x}_{\mathbf{i}}, p_{i}\right)$, where $\mathbf{x}_{\mathbf{i}}$ denotes a sample vector of dimension $V$ (one volume of the fMRI time series) and $p_{i}$ the corresponding onedimensional label (the EEG trial-by-trial modulation of a specific component).

In machine learning for fMRI data analysis it is common to use a linear model due to a typically high feature (i.e. voxel) to sample (i.e. scan) ratio. A standard linear model has the following form:

$$
p=y(\mathbf{x}, \mathbf{w})+\varepsilon
$$

where $y(\mathbf{x}, \mathbf{w})$ is the deterministic input-output mapping part and $\varepsilon$ accounts for the noise in the measurements. The deterministic mapping can be modelled as (Bishop, 2006):

$$
y(\mathbf{x}, \mathbf{w})=\mathbf{w}^{T} \phi(\tilde{\mathbf{x}})
$$

where $\phi: \mathfrak{R}^{V} \rightarrow \mathfrak{R}^{M}$ is a mapping (i.e. kernel) from $V$-dimensional space of $\mathbf{x}$ into an $M$-dimensional one and $\mathbf{w}$ is an $M$-dimensional vector of parameters.

Without loss of generality, we refer to the first functional run $\left(\mathbf{X}_{1}\right)$ as the training data set and subsequent runs as test set. Suppose that both the time courses of the voxels in the fMRI data and the simultaneously recorded EEG signals have zero mean. The choice of a linear kernel results in the following model: 


$$
y\left(\mathbf{X}_{1}, \mathbf{w}\right)=\mathbf{X}_{1} \mathbf{X}_{1}^{T} \mathbf{w}=\mathbf{K} \mathbf{w}
$$

with $\mathbf{w}\left(n_{1} \times 1\right)$ being the model weights vector and $\mathbf{K}=\mathbf{X}_{1} \mathbf{X}_{1}^{T}\left(n_{1} \times n_{1}\right)$ the linear kernel constructed from the starting training dataset $\mathbf{X}_{1}\left(n_{1} \times V\right)$.

The RVM training aims at finding an estimate of the posterior distribution of the weights $\mathbf{W}$. This posterior distribution can then be used to perform predictions on a new dataset (second functional run). Denoting with $\tilde{\mathbf{w}}$ the estimated posterior mean, then the prediction on the second functional run $\mathbf{X}_{2}\left(n_{2} \times V\right)$ can be expressed as:

$$
\tilde{p}_{2}=\mathbf{X}_{2} \mathbf{X}_{1}^{T} \tilde{\mathbf{w}}
$$

It is possible to express the prediction in terms of maps:

$$
\tilde{p}_{2}=\mathbf{X}_{2} \tilde{\mathbf{M}}
$$

with

$$
\tilde{\mathbf{M}}=\mathbf{X}_{1}^{T} \tilde{\mathbf{w}}
$$

where $\tilde{\mathbf{M}}(V \times 1)$ can be interpreted as a map of relative contribution of the different voxels to the final prediction" (De Martino et al., 2010, p.1106-1107).

\section{Evaluation of single trial modulations in multivariate predictions}

Considering the presence of the hemodynamic response, a high value of crosscorrelation between the predicted and actual response may simply reflect a good prediction of the overall response but not necessarily of the single-trial EEG fluctuations. This is especially evident when both the BOLD and EEG responses are highly stimuluslocked and thus closely fit the experimental protocol. To evaluate the contribution of the actual single trial EEG modulations to our predictions we employed three different procedures. Evaluations were only performed on the auditory ERP predictions, but the results can be extended to the visual ERP predictions.

First, we modelled the time courses of the RVM-based predictions post hoc, using a GLM analysis, in terms of "overall BOLD response" and "EEG modulations". The "overall BOLD response" was modelled as a standard fMRI predictor with constant 
amplitude and duration equal to the auditory cue, while the "EEG modulations" were obtained from the EEG-based predictor, informed by both amplitude and latency of testdata and orthogonalized with respect to the overall BOLD response. To evaluate the contribution of considering EEG information during learning in explaining EEG modulations in the test data, we compared the beta coefficients for RVM-based predictions obtained with and without EEG information (see Figure 4.7). The comparison was based on a two-way ANOVA for repeated measures with Subject (s1, s2, s3) and Training (EEG-informed, no-EEG) as factors. Significance values in Figure 4.7 ( $\mathrm{p}<0.05)$ refer to the factor "Training". In all cases, the factor "Subject" and the interaction term were not significant.

Second, we orthogonalized both fMRI time series (voxel-by-voxel) and EEGbased predictors with respect to the overall BOLD response to the experimental protocol. Voxel-by-voxel orthogonalization is necessary as methods of multivariate regression are currently limited to "learning" of single predictors. Multiple predictors are thus treated separately. Subsequently, training and testing was performed on the orthogonalized time series. Prediction accuracies were computed as the correlation of the EEG modulations predicted by the fMRI data and the real modulations of each run convolved with a canonical HRF response and orthogonalized with respect to the experimental protocol (Table 2 and Figure 4.8). The mean accuracies were computed by transforming the individual $r$-values into $z$-values and re-transforming the resulting mean $\mathrm{z}$ into an $\mathrm{r}$ value. Statistical significance on the single runs and on the mean was computed using $n$ equal to the minimum number of points present in each run $(n=450)$.

Third, we extracted single trial values from our prediction time courses and evaluated the coupling of predicted and real EEG modulations on a trial-by-trial basis (Debener et al., 2005). This procedure largely reduces the contribution of the overall hemodynamic response to the obtained correlation values. Furthermore, the significance of correlations is not inflated by the intrinsic autocorrelation of time courses. The prediction accuracies were computed as the cross-correlation of predicted (estimated as the mean over 3 TRs after each stimulus onset) and real single trial EEG modulations (Table 3). The mean accuracies were computed by transforming individual r-values into $z$-values and re-transforming the resulting mean $z$ into an $r$-value. Statistical significance is computed using $n$ equal to the minimum number of trials present in each run $(n=68)$. 


\subsubsection{Results of single trial modulation evaluations}

The presence of the hemodynamic response in both fMRI data and HRF convolved EEG data, makes it difficult to grasp how much the relatively small EEG modulations contribute to the predictions. Even though a high cross correlation was found between predicted and actual EEG responses, high correlation values were also obtained when learning was based on non-EEG informed predictors (constant amplitude and latency defined by the duration of the auditory cue) (see Table 1). This indicates that high values of correlation between predicted and actual response and their significance (computed on the total number of 450 points) simply reflect a good prediction of the hemodynamic response to the sound presentation but not necessarily of the single-trial EEG fluctuations. Therefore, we performed three analyses to evaluate the single trial modulations' contribution to the prediction accuracy.

Table 4.1 Comparison between EEG-informed and uniformed learning. Accuracies are computed as the correlation between fMRI-based predictions and real EEG signals after convolution with a canonical HRF response. Mean accuracies were computed by transforming individual r-values into $\mathrm{z}$-values and re-transforming the resulting mean $\mathrm{z}$ into an $\mathrm{r}$-value. Statistical significance is computed using $\mathrm{n}$ equal to the minimum number of points present in each run $(n=450)$.

\begin{tabular}{|c|c|c|c|c|c|c|c|}
\hline & & & EEG i & & & o EEG & \\
\hline \multirow{3}{*}{ S1 } & N1 & 0.46 & 0.54 & 0.61 & 0.51 & 0.55 & 0.60 \\
\hline & P2 & 0.59 & 0.63 & 0.71 & 0.59 & 0.62 & 0.62 \\
\hline & $\mathrm{SN}$ & 0.62 & 0.64 & 0.66 & 0.60 & 0.67 & 0.70 \\
\hline \multirow{3}{*}{ S2 } & N1 & 0.43 & 0.50 & 0.52 & 0.54 & 0.55 & 0.59 \\
\hline & P2 & 0.55 & 0.58 & 0.61 & 0.54 & 0.55 & 0.53 \\
\hline & $\mathrm{SN}$ & 0.61 & 0.64 & 0.67 & 0.66 & 0.60 & 0.62 \\
\hline \multirow{3}{*}{ S3 } & N1 & 0.45 & 0.49 & 0.59 & 0.50 & 0.55 & 0.58 \\
\hline & P2 & 0.48 & 0.56 & 0.48 & 0.48 & 0.55 & 0.63 \\
\hline & $\mathrm{SN}$ & 0.55 & 0.63 & 0.68 & 0.59 & 0.60 & 0.75 \\
\hline \multirow{3}{*}{ Mean } & N1 & \multicolumn{3}{|c|}{$\begin{aligned} & 0.51 \\
= & 5.4 \cdot 10^{-32}\end{aligned}$} & \multicolumn{3}{|c|}{$\begin{array}{c}0.55 \\
p=3 \cdot 10^{-37}\end{array}$} \\
\hline & P2 & \multicolumn{3}{|c|}{$\begin{aligned} & 0.58 \\
= & 4.1 \cdot 10^{-42}\end{aligned}$} & \multicolumn{3}{|c|}{$\begin{array}{c}0.57 \\
p=5.2 \cdot 10^{-42}\end{array}$} \\
\hline & SN & \multicolumn{3}{|c|}{$\begin{aligned} & 0.63 \\
= & 1.9 \cdot 10^{-51}\end{aligned}$} & \multicolumn{3}{|c|}{$\begin{array}{c}0.65 \\
p=4.1 \cdot 10^{-56}\end{array}$} \\
\hline
\end{tabular}


The results of a first analysis we conducted to examine the contribution of single trial EEG modulations are shown in Figure 4.7. With a post hoc GLM, we compared the beta values associated to the EEG modulations obtained with the EEG-based learning predictions and non-EEG based learning predictions for the same data (two-way ANOVA for repeated measures with "Subject" and "Training" as factors). Each prediction (N1, P2, SN) is illustrated in a different panel of Figure 4.7.
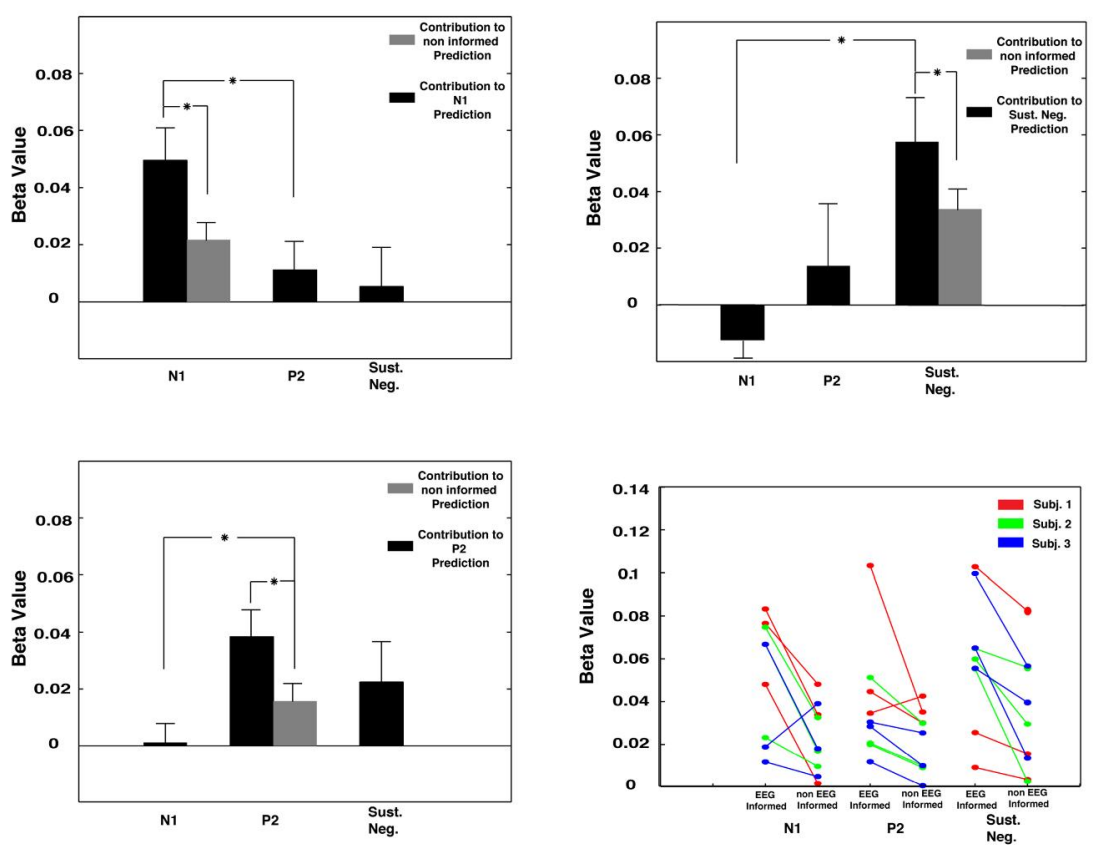

Figure 4.7 Statistical analysis performed on the prediction time course obtained using N1 (top left panel), P2 (bottom left panel) and SN (top right panel) information for training. Black bars represent the beta values associated with the orthogonalized single-trial EEG predictor in the case of EEG-informed training. Gray bars represent the beta values associated with the same predictor in the case of uninformed learning. Significant ( $p<$ 0.05 ) differences are highlighted (asterisk). The bottom right panel shows beta values associated with the relevant EEG modulations obtained from EEG-informed and non EEG-informed predictions for each individual run/subjects.

It can be observed that the beta values associated with the EEG information are significantly $(p<0.05)$ higher for the prediction obtained by the EEG informed training (black bars) compared to non-EEG-informed training (gray bars). Furthermore, the beta values are higher for the targeted EEG component (in most cases significantly at $p<0.05$, see Figure 4.7 for details). For example, when N1 information is used during training 
(top left panel), the beta value associated with the N1 variations in test data (orthogonalized with respect to the protocol) is not only significantly higher than the beta value obtained when analyzing the non-informed training prediction, but also higher than the beta's obtained for P2 (significantly at p < 0.03) and SN (although not significantly $\mathrm{p}=0.051$ ). Similar results are found for P2 (bottom left panel) and SN (top right panel) targeted predictions. The bottom right panel of figure 4.7 shows the single subject/run beta values associated to the three relevant peaks (N1, P2, SN) obtained with EEG informed and non EEG-informed predictions. These values indicate that the EEG informed training generally leads to higher beta values.

The results of a second analysis we conducted to examine the contribution of single trial EEG modulations are displayed in Table 4.2 and Figure 4.8. In this analysis the fMRI data were voxel-by-voxel orthogonalized to the experimental protocol, as were the EEG predictors. These data were then subjected to training and predicting. The results show that after the standard hemodynamic response to the stimulus was removed from the fMRI time-courses and EEG-based predictors, the correlations between predicted and actual responses in test data were much smaller, but still significant for P2 ( $c c=0.08, \mathrm{p}=0.03)$ and $\mathrm{SN}(\mathrm{cc}=0.11, \mathrm{p}=0.007)$ as can be seen in Figure 4.8 on the right side.
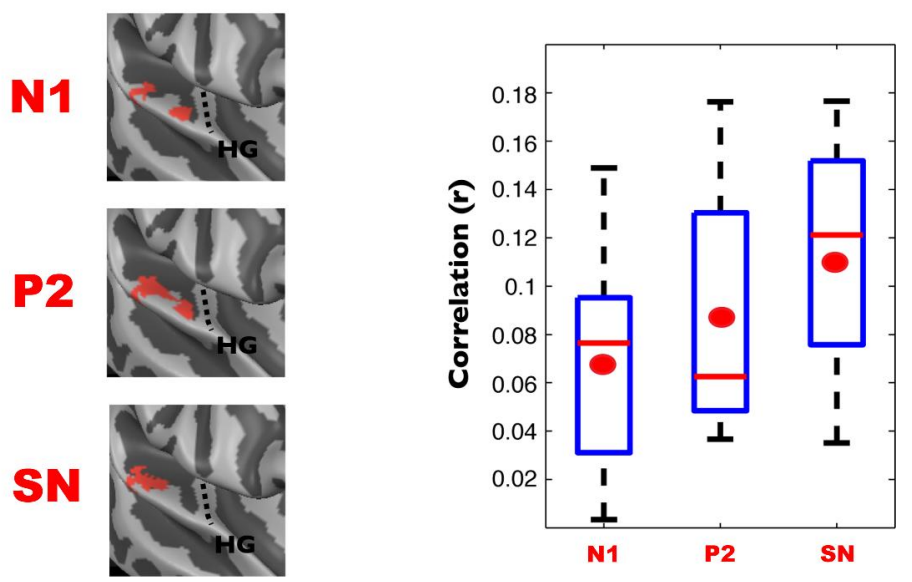

Figure 4.8 Results obtained from the prediction of the prominent ERP peaks of the early auditory IC after decorrelating the fMRI signals and predictors with respect to the experimental protocol. Left: Zoomed-in portion (right auditory cortex, group map) of the maps obtained using three different sources of ERP information during training (N1, P2 and sustained negativity). The maps are displayed after thresholding the single subjects to select the best $40 \%$ of the voxels. HG = Heschl's gyrus. Right: Box plots of the cross correlations between actual and predicted response for each ERP component (red dot = mean score, red line = median). 
The prediction accuracies at single-subject/run level are shown in Table 4.2, and show a variable pattern of significant and not significant accuracies. Generally, the SN prediction is most significant over single subjects/runs. The maps obtained from training using single trial information of the three auditory ERP peaks (N1, P2, SN; Figure 4.8) were similar to those obtained with non-orthogonalized data (Figure 4.4).

Table 4.2 Results of EEG-informed learning with fMRI time series orthogonalized with respect to the overall auditory response. Accuracies are computed as the correlation of the EEG modulations predicted by the fMRI and the real modulations of each run convolved with a canonical HRF response and orthogonalized with respect to the stimulation protocol. Mean accuracies were computed by transforming individual r-values into z-values and retransforming the resulting mean $\mathrm{z}$ into an $\mathrm{r}$-value. Statistical significance on the single runs and on the mean was computed using $n$ equal to the minimum number of points present in each run $(n=450)$.

\begin{tabular}{|c|c|c|c|c|}
\hline & & \multicolumn{3}{|c|}{ All EEG info } \\
\hline & & Run1 & Run2 & Run3 \\
\hline \multirow{3}{*}{ S1 } & N1 & $\begin{array}{c}0.10 \\
p=0.01\end{array}$ & $\begin{array}{c}0.04 \\
\mathrm{p}=0.16\end{array}$ & $\begin{array}{c}0.08 \\
p=0.04\end{array}$ \\
\hline & $\mathrm{P} 2$ & $\begin{array}{c}0.13 \\
p=0.002\end{array}$ & $\begin{array}{c}0.06 \\
p=0.09\end{array}$ & $\begin{array}{c}0.17 \\
\mathrm{p}=8.5 \cdot 10^{-5}\end{array}$ \\
\hline & SN & $\begin{array}{c}0.16 \\
\mathrm{p}=1.7 \cdot 10^{-4}\end{array}$ & $\begin{array}{c}0.09 \\
p=0.01\end{array}$ & $\begin{array}{c}0.12 \\
p=0.003\end{array}$ \\
\hline \multirow{3}{*}{ S2 } & N1 & $\begin{array}{c}0.09 \\
p=0.02\end{array}$ & $\begin{array}{c}0.01 \\
p=0.3\end{array}$ & $\begin{array}{c}0.07 \\
\mathrm{p}=0.05\end{array}$ \\
\hline & $\mathrm{P} 2$ & $\begin{array}{c}0.04 \\
p=0.16\end{array}$ & $\begin{array}{c}0.04 \\
p=0.16\end{array}$ & $\begin{array}{c}0.03 \\
p=0.2\end{array}$ \\
\hline & $\mathrm{SN}$ & $\begin{array}{c}0.07 \\
\mathrm{p}=0.05\end{array}$ & $\begin{array}{c}0.07 \\
p=0.05\end{array}$ & $\begin{array}{c}0.03 \\
p=0.2\end{array}$ \\
\hline \multirow{3}{*}{ S3 } & N1 & $\begin{array}{c}0.003 \\
p=0.4\end{array}$ & $\begin{array}{c}0.14 \\
\mathrm{p}=7.6 \cdot 10^{-4}\end{array}$ & $\begin{array}{c}0.03 \\
p=0.2\end{array}$ \\
\hline & P2 & $\begin{array}{c}0.08 \\
p=0.04\end{array}$ & $\begin{array}{c}0.12 \\
p=0.003\end{array}$ & $\begin{array}{c}0.05 \\
p=0.11\end{array}$ \\
\hline & $\mathrm{SN}$ & $\begin{array}{c}0.17 \\
\mathrm{p}=8.5 \cdot 10^{-5}\end{array}$ & $\begin{array}{c}0.13 \\
p=0.002\end{array}$ & $\begin{array}{c}0.14 \\
\mathrm{p}=7.6 \cdot 10^{-4}\end{array}$ \\
\hline \multirow{3}{*}{ Mean } & N1 & \multicolumn{3}{|c|}{$\begin{array}{c}0.06 \\
p=0.07\end{array}$} \\
\hline & $\mathrm{P} 2$ & \multicolumn{3}{|c|}{$\begin{array}{c}0.08 \\
p=0.03\end{array}$} \\
\hline & SN & \multicolumn{3}{|c|}{$\begin{array}{c}0.11 \\
p=0.007\end{array}$} \\
\hline
\end{tabular}


Finally, the predictions obtained from the EEG-informed training on both nonorthogonalized (Figure 4.4 and Table 1) and orthogonalized data (Figure 4.8 and Table 2) were evaluated in terms of trial-by-trial correlations. Estimates of single trial EEG modulations were obtained by averaging the signal values at three TRs after each stimulus onset for both the RVM-based predicted response and the real EEG modulations. The results of this analysis are reported in Table 3 ( $p$-values were computed using the minimum number of trials in each prediction $[n=68]$ ). This table also reports the correlations obtained in case of non-EEG informed training. It can be seen that - although variable across runs and subjects - correlation values obtained with EEG informed training (original and orthogonal data) are generally higher than those obtained with non-EEG informed training.

Overall, these three different analyses of the auditory event-related responses indicate that considering the information of single-trial modulations in training data provides a small but significant improvement in predicting modulations of corresponding EEG responses in test data. 
Table 4.3 Comparison between EEG informed learning with original and orthogonalized fMRI time series and non-EEG informed training. Accuracies are computed as the correlation of predicted (estimated as the mean over 3 TRs after each stimulus onset) and real single trial EEG modulations. Mean accuracies were computed by transforming individual r-values into $\mathrm{z}$-values and re-transforming the resulting mean $\mathrm{z}$ into an r-value. Statistical significance is computed using $n$ equal to the minimum number of trials present in each run $(n=68)$.

\begin{tabular}{|c|c|c|c|c|c|c|c|c|c|c|}
\hline & \multicolumn{3}{|c|}{$\begin{array}{c}\text { All EEG info } \\
\text { (non orth. data) }\end{array}$} & \multicolumn{3}{|c|}{$\begin{array}{c}\text { All EEG info } \\
\text { (orth. data) }\end{array}$} & \multicolumn{3}{|c|}{ No EEG info } \\
\hline & & Run1 & Run2 & Run3 & Run1 & Run2 & Run3 & Run1 & Run2 & Run3 \\
\hline \multirow{9}{*}{ S1 } & & 0.23 & 0.21 & 0.24 & 0.16 & 0.21 & 0.19 & & 0.14 & 0.004 \\
\hline & N1 & $\mathrm{p}=$ & $\mathrm{p}=$ & $\mathrm{p}=$ & $\mathrm{p}=$ & $\mathrm{p}=$ & $\mathrm{p}=$ & 0.02 & $\mathrm{p}=$ & $\mathrm{p}=$ \\
\hline & & 0.02 & 0.03 & 0.02 & 0.07 & 0.03 & 0.05 & & 0.1 & 0.5 \\
\hline & & 0.17 & 0.12 & 0.13 & 0.24 & 0.11 & 0.12 & 0.01 & 0.05 & 0.11 \\
\hline & $\mathrm{P} 2$ & $\mathrm{p}=$ & $\mathrm{p}=$ & $\mathrm{p}=$ & $\mathrm{p}=$ & $\mathrm{p}=$ & $\mathrm{p}=$ & $\mathrm{p}=$ & $\mathrm{p}=$ & $\mathrm{p}=$ \\
\hline & & 0.07 & 0.14 & 0.12 & 0.02 & 0.16 & 0.14 & 0.4 & 0.3 & 0.16 \\
\hline & & 0.30 & 0.11 & 0.14 & 0.22 & 0.17 & 0.09 & 0.11 & 0.09 & 0.04 \\
\hline & $\mathrm{SN}$ & $\mathrm{p}=$ & $\mathrm{p}=$ & $\mathrm{p}=$ & $\mathrm{p}=$ & $\mathrm{p}=$ & $\mathrm{p}=$ & $\mathrm{p}=$ & $\mathrm{p}=$ & $\mathrm{p}=$ \\
\hline & & 0.005 & 0.16 & 0.1 & 0.02 & 0.07 & 0.2 & 0.16 & 0.2 & 0.4 \\
\hline \multirow{9}{*}{ S2 } & & 0.19 & 0.15 & 0.12 & 0.17 & 0.16 & 0.16 & 0.01 & 0.05 & 0.006 \\
\hline & N1 & $\mathrm{p}=$ & $\mathrm{p}=$ & $\mathrm{p}=$ & $\mathrm{p}=$ & $\mathrm{p}=$ & $\mathrm{p}=$ & $\mathrm{p}=$ & $\mathrm{p}=$ & $\mathrm{p}=$ \\
\hline & & 0.05 & 0.1 & 0.14 & 0.07 & 0.07 & 0.07 & 0.4 & 0.33 & 0.5 \\
\hline & & 0.09 & 0.17 & 0.05 & 0.12 & 0.15 & 0.14 & 0.09 & 0.13 & 0.21 \\
\hline & P2 & $p=$ & $p=$ & $p=$ & $p=$ & $\mathrm{p}=$ & $p=$ & $p=$ & $\mathrm{p}=$ & $\mathrm{p}=$ \\
\hline & & 0.2 & 0.07 & 0.33 & 0.14 & 0.1 & 0.1 & 0.2 & 0.12 & 0.03 \\
\hline & & 0.17 & 0.19 & 0.33 & 0.22 & 0.12 & 0.27 & 0.01 & 0.09 & 0.09 \\
\hline & $\mathrm{SN}$ & $\mathrm{p}=$ & $\mathrm{p}=$ & $\mathrm{p}=$ & $\mathrm{p}=$ & $\mathrm{p}=$ & $\mathrm{p}=$ & $\mathrm{p}=$ & $\mathrm{p}=$ & $\mathrm{p}=$ \\
\hline & & 0.07 & 0.05 & 0.001 & 0.02 & 0.14 & 0.009 & 0.4 & 0.2 & 0.2 \\
\hline \multirow{9}{*}{ S3 } & & 0.08 & 0.13 & 0.18 & 0.14 & 0.15 & 0.19 & 0.0006 & 0.01 & 0.01 \\
\hline & N1 & $\mathrm{p}=$ & $\mathrm{p}=$ & $\mathrm{p}=$ & $\mathrm{p}=$ & $\mathrm{p}=$ & $p=$ & $\mathrm{p}=$ & $\mathrm{p}=$ & $\mathrm{p}=$ \\
\hline & & 0.24 & 0.12 & 0.06 & 0.1 & 0.1 & 0.05 & 0.5 & 0.4 & 0.4 \\
\hline & & 0.19 & 0.35 & 0.09 & 0.33 & 0.11 & 0.09 & 0.01 & 0.01 & 0.0003 \\
\hline & P2 & $\mathrm{p}=$ & $\mathrm{p}=$ & $\mathrm{p}=$ & $\mathrm{p}=$ & $\mathrm{p}=$ & $\mathrm{p}=$ & $\mathrm{p}=$ & $\mathrm{p}=$ & $\mathrm{p}=$ \\
\hline & & 0.05 & 0.001 & 0.2 & 0.001 & 0.16 & 0.2 & 0.4 & 0.4 & 0.5 \\
\hline & & 0.14 & 0.09 & 0.24 & 0.13 & 0.18 & 0.18 & 0.01 & 0.14 & 0.07 \\
\hline & $\mathrm{SN}$ & $\mathrm{p}=$ & $p=$ & $\mathrm{p}=$ & $\mathrm{p}=$ & $\mathrm{p}=$ & $\mathrm{p}=$ & $\mathrm{p}=$ & $\mathrm{p}=$ & $\mathrm{p}=$ \\
\hline & & 0.1 & 0.2 & 0.02 & 0.13 & 0.06 & 0.06 & 0.4 & 0.1 & 0.2 \\
\hline \multirow{3}{*}{ Mean } & N1 & \multicolumn{3}{|c|}{$\begin{array}{c}0.17 \\
p=0.07\end{array}$} & \multicolumn{3}{|c|}{$\begin{array}{c}0.17 \\
p=0.07\end{array}$} & \multicolumn{3}{|c|}{$\begin{array}{c}0.03 \\
p=0.3\end{array}$} \\
\hline & P2 & \multicolumn{3}{|c|}{$\begin{array}{c}0.15 \\
p=0.1\end{array}$} & \multicolumn{3}{|c|}{$\begin{array}{c}0.16 \\
p=0.07\end{array}$} & \multicolumn{3}{|c|}{$\begin{array}{c}0.07 \\
p=0.2\end{array}$} \\
\hline & SN & \multicolumn{3}{|c|}{$\begin{array}{c}0.19 \\
p=0.05\end{array}$} & \multicolumn{3}{|c|}{$\begin{array}{c}0.18 \\
p=0.06\end{array}$} & \multicolumn{3}{|c|}{$\begin{array}{c}0.07 \\
p=0.2\end{array}$} \\
\hline
\end{tabular}


Ackermann RF, Finch DM, Babb TL, Engel J, Jr. (1984) Increased glucose metabolism during longduration recurrent inhibition of hippocampal pyramidal cells. J Neurosci 4:251-264.

Alivisatos B, Petrides M (1997) Functional activation of the human brain during mental rotation. Neuropsychologia 35:111-118.

Bhattacharya J, Petsche H (2005) Drawing on mind's canvas: differences in cortical integration patterns between artists and non-artists. Human Brain Mapping 26:1-14.

Bishop CM (2006) Pattern Recognition and Machine Learning. New York: Springer.

Cabeza R, Nyberg L (2000) Imaging cognition II: An empirical review of 275 PET and fMRI studies. J Cogn Neurosci 12:1-47.

Cooper NR, Croft RJ, Dominey SJ, Burgess AP, Gruzelier JH (2003) Paradox lost? Exploring the role of alpha oscillations during externally vs. internally directed attention and the implications for idling and inhibition hypotheses. International Journal of Psychophysiology 47:65-74.

Cooper R, Osselton JW, Shaw JC (1980) EEG technology, 3rd Edition. London: Butterworths.

Creutzfeldt OD, Watanabe S, Lux HD (1966) Relations between EEG phenomena and potentials of single cortical cells. I. Evoked responses after thalamic and erpicortical stimulation. Electroencephalogr Clin Neurophysiol 20:1-18.

Dale AM, Halgren E (2001) Spatiotemporal mapping of brain activity by integration of multiple imaging modalities. Curr Opin Neurobiol 11:202-208.

De Martino F, Valente G, de Borst AW, Esposito F, Roebroeck A, Goebel R, Formisano E (2010) Multimodal imaging: an evaluation of univariate and multivariate methods for simultaneous EEG/fMRI. Magn Reson Imaging 28: 1104-1112.

Debener S, Ullsperger M, Siegel M, Engel AK (2006) Single-trial EEG-fMRI reveals the dynamics of cognitive function. Trends Cogn Sci 10:558-563.

Debener S, Ullsperger M, Siegel M, Fiehler K, von Cramon DY, Engel AK (2005) Trial-by-trial coupling of concurrent electroencephalogram and functional magnetic resonance imaging identifies the dynamics of performance monitoring. J Neurosci 25:11730-11737.

Debener S, Strobel A, Sorger B, Peters J, Kranczioch C, Engel AK, Goebel R (2007) Improved quality of auditory event-related potentials recorded simultaneously with 3-T fMRI: removal of the ballistocardiogram artefact. Neuroimage 34:587-597.

Delorme A, Makeig S (2004) EEGLAB: an open source toolbox for analysis of single-trial EEG dynamics including independent component analysis. Journal of Neuroscience Methods 134:9-21.

Eichele T, Specht K, Moosmann M, Jongsma ML, Quiroga RQ, Nordby H, Hugdahl K (2005) Assessing the spatiotemporal evolution of neuronal activation with single-trial eventrelated potentials and functional MRI. Proc Natl Acad Sci U S A 102:17798-17803.

Formisano E, De Martino F, Valente G (2008) Multivariate analysis of fMRI time series: classification and regression of brain responses using machine learning. Magn Reson Imaging 26:921934. 
Formisano E, Pepino A, Bracale M, Di Salle F, Saulino C, Marciano E (1998) Localisation and characterisation of auditory perception through Functional Magnetic Resonance Imaging. Technol Health Care 6:111-123.

Formisano E, Linden DE, Di Salle F, Trojano L, Esposito F, Sack AT, Grossi D, Zanella FE, Goebel R (2002) Tracking the mind's image in the brain I: time-resolved fMRI during visuospatial mental imagery. Neuron 35:185-194.

Friederici AD, Hahne A, von Cramon DY (1998) First-pass versus second-pass parsing processes in a Wernicke's and a Broca's aphasic: electrophysiological evidence for a double dissociation. Brain Lang 62:311-341.

Friston KJ, Fletcher P, Josephs O, Holmes A, Rugg MD, Turner R (1998) Event-related fMRI: characterizing differential responses. Neuroimage 7:30-40.

Goebel R, Esposito F, Formisano E (2006) Analysis of functional image analysis contest (FIAC) data with brainvoyager QX: From single-subject to cortically aligned group general linear model analysis and self-organizing group independent component analysis. Human Brain Mapping 27:392-401.

Harel N, Ugurbil K, Uludag K, Yacoub E (2006) Frontiers of brain mapping using MRI. J Magn Reson Imaging 23:945-957.

Holz EM, Glennon M, Prendergast K, Sauseng P (2010) Theta-gamma phase synchronization during memory matching in visual working memory. Neuroimage.

Ishai A, Ungerleider LG, Haxby JV (2000) Distributed neural systems for the generation of visual images. Neuron 28:979-990.

Ishai A, Haxby JV, Ungerleider LG (2002) Visual imagery of famous faces: effects of memory and attention revealed by fMRI. Neuroimage 17:1729-1741.

Kaiser J, Lutzenberger W (2003) Induced gamma-band activity and human brain function. Neuroscientist 9:475-484.

Knauff M, Kassubek J, Mulack T, Greenlee MW (2000) Cortical activation evoked by visual mental imagery as measured by fMRI. Neuroreport 11:3957-3962.

Lamm C, Windischberger C, Leodolter U, Moser E, Bauer H (2001) Evidence for premotor cortex activity during dynamic visuospatial imagery from single-trial functional magnetic resonance imaging and event-related slow cortical potentials. Neuroimage 14:268-283.

Logothetis NK (2008) What we can do and what we cannot do with fMRI. Nature 453:869-878.

Mellet E, Tzourio N, Crivello F, Joliot M, Denis M, Mazoyer B (1996) Functional anatomy of spatial mental imagery generated from verbal instructions. Journal of Neuroscience 16:6504-6512.

Mellet E, Briscogne S, Tzourio-Mazoyer N, Ghaem O, Petit L, Zago L, Etard O, Berthoz A, Mazoyer B, Denis M (2000) Neural correlates of topographic mental exploration: the impact of route versus survey perspective learning. Neuroimage 12:588-600.

Niazy RK, Beckmann CF, Iannetti GD, Brady JM, Smith SM (2005) Removal of FMRI environment artifacts from EEG data using optimal basis sets. Neuroimage 28:720-737.

O'Craven KM, Kanwisher N (2000) Mental imagery of faces and places activates corresponding stiimulus-specific brain regions. Journal of Cognitive Neuroscience 12:1013-1023. 
Petsche H, Kaplan S, von Stein A, Filz O (1997) The possible meaning of the upper and lower alpha frequency ranges for cognitive and creative tasks. International Journal of Psychophysiology 26:77-97.

Raichle ME (1998) Behind the scenes of functional brain imaging: a historical and physiological perspective. Proc Natl Acad Sci U S A 95:765-772.

Ritter P, Villringer A (2006) Simultaneous EEG-fMRI. Neurosci Biobehav Rev 30:823-838.

Rugg MD, Venables PH (1980) EEG correlates of the acquisition of high- and low-imagery words. Neuroscience Letters 16:67-70.

Sack AT, Camprodon JA, Pascual-Leone A, Goebel R (2005) The dynamics of interhemispheric compensatory processes in mental imagery. Science 308:702-704.

Sack AT, Sperling JM, Prvulovic D, Formisano E, Goebel R, Di Salle F, Dierks T, Linden DE (2002) Tracking the mind's image in the brain II: transcranial magnetic stimulation reveals parietal asymmetry in visuospatial imagery. Neuron 35:195-204.

Scheeringa R, Petersson KM, Oostenveld R, Norris DG, Hagoort P, Bastiaansen MC (2009) Trial-bytrial coupling between EEG and BOLD identifies networks related to alpha and theta EEG power increases during working memory maintenance. Neuroimage 44:1224-1238.

Talairach J, Tournoux P (1988) Co-planar Stereotaxic Atlas of the Human Brain: 3-Dimensional Proportional System - an Approach to Cerebral Imaging. New York: Thieme Medical Publishers.

Tallon-Baudry C, Bertrand O, Peronnet F, Pernier J (1998) Induced gamma-band activity during the delay of a visual short-term memory task in humans. J Neurosci 18:4244-4254.

Tipping ME (2001) Sparse Bayesian learning and the relevance vector machine. Journal of Machine Learning Research 1:211-244.

Trojano L, Trojano L, Linden DEJ, Formisano E, Goebel R, Sack AT, Di Salle F (2004) What clocks tell us about the neural correlates of spatial imagery. European Journal of Cognitive Psychology 16:653.

Ugurbil K, Toth L, Kim DS (2003) How accurate is magnetic resonance imaging of brain function? Trends Neurosci 26:108-114. 
Chapter 4

94 


\section{Chapter 5}

\section{Effective connectivity of the mental imagery network}

\section{Abstract}

Imagining an object or scene is accompanied by the activation of a large network of brain regions. Previous neuroimaging studies have mostly focused on the specific role of each of the nodes of this network (i.e. the brain regions), while the functional integration within this network remains largely unknown. The functional integration of a distributed brain network can be defined by measuring the effective connectivity, the influence one neuronal system exerts over another. Granger Causality Mapping (GCM) is one method that allows investigating effective connectivity in a data driven manner. In this study we use GCM to examine a full brain mental imagery network that is activated during a scene imagery task. The results reveal a central role of the mesial superior frontal gyrus, which connects to all content-specific regions in parietal, temporal and occipital cortex. Based on these results we propose a neuro-anatomical model of scene imagery in which the integration of information necessary for performing the mental imagery task is subserved by the interaction of frontal regions with remote parietal regions for retrieval and representation of the spatial content of the scene and with occipital-temporal regions for detailed visual representation of the objects. The inclusion of previous electroencephalography findings within this model suggests the synchronization of neuronal oscillation in the theta and beta bands as possible mediating neuronal mechanisms.

\section{Based on}

A.W. de Borst, A. Sack, E. Formisano and A. Roebroeck. Working Title: "Effective connectivity of the mental imagery network". 


\subsection{Introduction}

Mental imagery is a cognitive construct that we use effortlessly on a daily basis, even though its neuro-biological basis is a complex system with a multitude of nodes (i.e. brain regions) and interaction patterns. So far, neuroimaging studies have investigated the specificity of involvement of a subset of these regions, during tasks that focused on a particular aspect of mental imagery. These studies have shown that during tasks that involve spatial mental manipulations specific regions of the dorsal visual processing stream, such as the superior parietal lobe, are activated (a.o. Mellet et al., 1996; Alivisatos and Petrides, 1997; Knauff et al., 2000; Mellet et al., 2000a; Formisano et al., 2002; Trojano et al., 2004). During tasks that involve processing of imagined objects category-specific regions in ventral temporal cortex are activated (Ishai et al., 2000; O'Craven and Kanwisher, 2000; Ishai et al., 2002). In addition to this, a set of regions was identified that contributes to non-content specific mental operations such as working memory. These regions include the right middle frontal cortex, mesial superior frontal cortex and premotor cortex (for an overview see Cabeza and Nyberg, 2000; Repovs and Baddeley, 2006).

Identifying which regions are specialized in processing specific facets of mental imagery is only part of understanding its functional organization. Equally essential is the investigation of the functional integration between regions, its connectivity (Friston, 1994). The functional integration between brain regions can be studied by assessing the functional or the effective connectivity. Functional connectivity is defined as the correlation between two distant neurophysiological sites. A correlation alone however, does not explain any underlying causal relationships. Therefore, functional integration can be better understood in the context of effective connectivity. Effective connectivity concerns modelling interactions that explain activity and timing relationships between neurons or neuronal populations in a directed manner, i.e. the influence one neuronal system exerts over another (Friston, 1994). A number of different approaches or models have been suggested to investigate effective connectivity in the context of functional neuroimaging, such as Structural Equation Modelling (SEM; McIntosh et al., 1994; Buchel and Friston, 1997), Granger Causality Mapping (GCM; Roebroeck et al., 2005) or Dynamic Causal Modelling (DCM; Friston et al., 2003; Stephan et al., 2008). Each of these models has its own benefits, and its suitability depends on the research questions and the experimental design.

So far, only two studies have investigated the dynamic causal interactions between a sub-set of brain regions within the fronto-parietal mental imagery network. A study by Mechelli et al. (2004) investigated influences between prefrontal, parietal and 
several ventral temporal regions during perception and imagery of houses, faces and chairs with DCM. The results showed that category-selective activation in ventraltemporal cortex is mediated by content-specific backward projections from prefrontal cortex during imagery, rather than bottom-up projections from visual areas as during perception. Sack et al. (2008) used GCM to show that during spatial imagery, information processing is organized top-down from premotor cortex to parietal cortex at two different stages during their task.

The use of GCM allows for the investigation of effective connectivity in a datadriven manner. It uses vector autoregressive moddeling of the fMRI time-series, in which the amount that past values of region $(x)$ contribute to the prediction of the current value of voxel $(y)$ is quantified. Therefore, directed influences from and to any region in the brain can be uncovered. Since GCM exploits temporal information in the fMRI time series, it is particularly suited to study complex tasks, such as mental imagery, that are known to evoke a cascade of neural activations in different brain regions at a temporal scale of several hundreds of milliseconds. Previous studies suggest that such temporal scale can be tackled with fMRI - despite the sluggishness and variability of the hemodynamic response (Menon and Kim, 1999; Formisano and Goebel, 2003; Roebroeck et al., 2005).

The present study provides novel information on the cortical interactions during mental imagery at two different levels. First of all, our behaviourally controlled task requires the participants to imagine objects as well as their spatial lay-out. Where previous studies focussed on either the ventral "what" stream (Mechelli et al., 2004) or the dorsal "where" stream (Sack et al., 2008), we are interested in investigating network interactions between all regions that are active during visual mental imagery. Secondly, by using GCM, we can include all these active regions in the effective connectivity analysis, since GCM does not require any a-priori assumptions on the network nodes. Simirlarly, no a-priori assumptions on the directions of connectivity have to be made. Since limited research has been performed on dynamic interactions between regions within the mental imagery network, a data-driven approach is most suitable to find, perhaps unexpected, effective connections.

With the combination of our experimental design and a data-driven effective connectivity method we strive to uncover the cortical interactions of the full visual mental imagery network, bringing us closer to understanding the complexity of mental imagery as used in real-life situations. 


\subsection{Methods}

\subsubsection{Participants}

Ten healthy right- handed volunteers (8 female, mean age 22.2 years; range 18-26) participated in the fMRI study. All participants had normal or corrected-to-normal vision. The local ethical committee approved the study and participants gave their written informed consent before participation.

\subsubsection{Task and stimuli}

Twenty-seven digital colour photographs were used as stimuli (screen and picture resolution 1024 by 768 pixels). Three photographs (see example in Figure 5.1, for colour version see Figure 2.1) depicted the interior of a room and comprised several pieces of furniture. These photographs defined the visual imagery content and were solely used during a training phase. Twelve photographs (targets) were fragments of the three original scenes (four per scene), containing a minimum of two partly visible objects. The other twelve photographs (targets) were these same images mirrored across the horizontal axis (see Figure 5.1). The default image was a grey background ( $50 \%$ black, $50 \%$ white). All photographs contained a centralized white fixation cross and were presented at a 24 by $30^{\circ}$ visual angle.

\begin{tabular}{|c|c|c|c|c|c|}
\hline Pre-scanning & $\begin{array}{c}\text { Task } \\
\text { duringfMRI }\end{array}$ & Sound & Imagery & Target & $\begin{array}{l}\text { Response \& } \\
\text { Fixation }\end{array}$ \\
\hline $\begin{array}{c}\text { Learning } \\
\text { scenes }\end{array}$ & $\begin{array}{c}\text { Duration } \\
(\mathrm{msec})\end{array}$ & 1000 & $6000(\mathrm{av})$ & 2500 & $10000(\mathrm{av})$ \\
\hline
\end{tabular}

Figure 5.1 Stimulus protocol. Schematic overview of the temporal course of one trial in milliseconds (ms). (av) = on average.

The fMRI experiment consisted of 54 (3 associations x 18 repetitions) trials. As depicted 
in Figure 5.1, each trial of the experiment started with an auditory cue. The three auditory stimuli were one second (s) sound clips of a female speaker uttering "photo one", "photo two" or "photo three". During the subsequent interval participants imagined the scene that they previously had learned to associate with the cue. After a variable delay $(5,6$, or $7 \mathrm{~s}$, average $=6 \mathrm{~s})$ a mirrored or non-mirrored fragment of the scene (50-50\% distribution) was visually presented as a target. After each target participants pressed a button with their right middle or index finger to indicate whether the fragment matched the imagined scene, or was mirrored. All trials ended with a fixation period ( $8-12 \mathrm{~s}$ ) during which the participants performed no task. The interval lengths were pseudo-randomized over trials and the occurrence of each period length was equally distributed over the three types of trials (scene one, two and three) and type of target picture. The trials (e.g. the order of the tones and target pictures) were pseudorandomized.

Prior to fMRI measurements, participants were trained on imagining three scenes, associated with three auditory cues. The training session consisted of 24 randomized trials of simultaneous presentation of the auditory stimulus and the corresponding visual scene (5 s), followed by a fixation period (6 s) during which the participants imagined the scene. After training, participants received task instructions and were asked to vividly visualize the correct scene after each cue in the main experiment, while fixating at the fixation cross.

\subsubsection{Data acquisition}

A 3T Siemens MR (head) scanner (MAGNETOM Allegra, Siemens Medical Systems, Erlangen, Germany) was used for imaging. Functional scans were acquired with a Gradient Echo Echo Planar Imaging sequence with a Repetition Time (TR) of 1500 ms and an Echo Time (TE) of $30 \mathrm{~ms}$. For each of the two functional runs 370 volumes were acquired composed of 23 slices (matrix $=64 \times 64$, voxel dimensions $=3 \times 3 \times 4.5 \mathrm{~mm} 3$, interslice time $=65 \mathrm{~ms}$, flip angle $=90^{\circ}$ ). Between the two functional runs high resolution T1- weighted structural images of the whole brain were acquired (3 subjects: MPRAGE 192 slices, matrix $=256 \times 256$, voxel dimensions $=1 \times 1 \times 1 \mathrm{~mm} 3, \mathrm{TR}=2300 \mathrm{~ms}, \mathrm{TE}=3.93$ ms, flip angle $=12^{\circ} ; 7$ subjects: MDEFT 176 slices, matrix $=256 \times 256$, voxel dimensions $=$ $1 \times 1 \times 1 \mathrm{~mm} 3, \mathrm{TR}=7.92 \mathrm{~ms}, \mathrm{TE}=2.40 \mathrm{~ms}$, flip angle $=15^{\circ}$ ). 


\subsubsection{Data analyses}

\section{Preprocessing}

All functional MRI data were analysed using fMRI analysis and visualisation software BrainVoyager QX (Brain Innovation B.V., Maastricht, The Netherlands). Functional data were 3D motion corrected (trilinear interpolation), corrected for slice scan time differences and temporally filtered (high pass: 5 cycles/run cut-off). The data were spatially smoothed with a $6 \mathrm{~mm}$ Gaussian filter. The anatomical data were corrected for intensity inhomogeneity (Goebel et al., 2006) and transformed into Talairach space (Talairach and Tournoux, 1988). The functional data were then aligned with the anatomical data and transformed into the same space, to create $4 \mathrm{D}$ volume time-courses (VTCs). The anatomical data were used for surface reconstruction of both hemispheres in all subjects (description of cortex segmentation in Goebel et al., 2006).

\section{Latency mapping}

Statistical modelling of the time-courses was based on a general linear model with a design matrix implementing a deconvolution analysis. An estimate of the hemodynamic response at each TR $(\mathrm{TR}=1.5 \mathrm{~s})$ during a $19.5 \mathrm{~s}$ window after trial onset was obtained using a separate stick predictor. The group map depicting the spatio-temporal pattern of BOLD activation (Figure 5.2) was based on the concatenation of single-subject deconvolution matrices (fixed effect GLM). In this map, a location was considered to be active during mental imagery if the significance of the statistical contrast including the first five stick predictors (preceding the onset of the target) was below the voxel-wise threshold of $\mathrm{p}$ (uncorrected) < 0.0001 . Maps of BOLD response latency were obtained by colour coding significantly active voxels according to the earliest stick predictor for which the beta weights reached significance (i.e. a dark green colour indicates a significant beta weight for the stick predictor corresponding to the first TR after sound onset, a lighter green to second TR, etc.).

All regions that were significantly activated $(R>0.36)$ in the single subject deconvolution maps were considered as Regions of Interest (ROIs) (cluster threshold 50 voxels). Only ROIs that were activated in all of the subjects were used for the subsequent Granger Causality Mapping (GCM) analyses. 


\section{Granger Causality Mapping}

Granger causality is an analysis technique that measures effective connectivity between brain regions using vector autoregressive (VAR) models. It rests on the principle that when we can predict the current value of a time-series $x$ better with the past values of time-series $y$ and $x$ than we can with the past of $x$ alone, we say that $y$ granger causes $x$ (Roebroeck et al., 2005). By defining $x$, the seed time-series, as a cluster of voxels (region of interest, ROI) and the time-series y as all grey matter voxels, GCM can be used as a data-driven measure to explore any causal relationships between a ROI and any other grey matter voxel. The influence measure Fx.y consist of three components; Fx $\rightarrow y$ (linear influence from $x$ to $y$ ), Fy $\rightarrow x$ (linear influence from $y$ to $x$ ) and Fx.y (the instantaneous correlation between $x$ and $y$ ). In order to give a most unbiased view of the directed interactions a difference map (dGCM) can be calculated as the subtraction of $\mathrm{Fy} \rightarrow \mathrm{x}$ from $\mathrm{Fx} \rightarrow \mathrm{y}$. In such a map the influence from reference region $\mathrm{x}$ to other regions is reflected in positive values and the influence from other regions to reference region $\mathrm{x}$ is indicated in negative values. All maps can be calculated per individual, but also random effects (RFX) Granger Causality maps can be calculated on a group of subjects. For the mathematical details of GCM see Roebroeck et al. (2005).

The following GCM procedure was employed on the pre-processed fMRI data of this study. Since the mental imagery task started with an auditory cue, the first cortical region to be activated was the primary auditory cortex. This region was chosen as a first seed region for the individual GCMs. After inspection of this map a new set of regions (regions on which the auditory cortex exerts influence) was chosen. These maps were again inspected in the same manner, and new regions were selected etc. This iterative process gave rise to a data-driven selection of regions. Of this set of regions their activation during the task was checked with the results of the GLM analysis (Figure 5.2), to exclude task-irrelevant regions. Only those voxels that were significantly activated during the task were selected as a ROI. For each region the following verification was done: if the GCM map of seed region x shows influence to region y, then the GCM map of seed region y should show influence from region $\mathrm{x}$. Only if this criterion was met a region was included in the results. Furthermore, we only included a directed influence between two regions if it was consistent across all subjects. Three subjects had to be excluded from the analyses due to a reduced number of data-points (1 subject) or a particularly constrained network of brain regions active during the task (2 subjects). Using these three criteria we came to a map of directed influence between 9 regions. A random effects (RFX) GCM group analysis was then performed on 7 subjects. To display 
Chapter 5

the network nodes in a clear manner, Matlab function Biograph (The MatWorks Inc.) was used to create a graph based on the network connections (Figure 5.4). 


\subsection{Results}

All fMRI results are solely based on the imagery period during the trials, excluding target picture information.

\subsubsection{Behavioural results}

Participants $(\mathrm{N}=10)$ responded correctly on 92 percent $(\mathrm{SD}=10.2)$ of the non- mirrored trials and 88 percent $(S D=11.3)$ of the mirrored trials with an average reaction time of $1823 \mathrm{~ms}(\mathrm{SD}=642.3)$ for non-mirrored and $2008 \mathrm{~ms}(\mathrm{SD}=808.6)$ for mirrored trials. A paired sampled $t$-test showed that responses were significantly faster $(\mathrm{t}(9)=-2.32, \mathrm{p}<$ $0.046)$ and more accurate $(\mathrm{t}(9)=2.34, \mathrm{p}<0.044)$ during the non- mirrored trials.

\subsubsection{General fMRI results}

Latency mapping of fMRI data (Figure 2.2) showed an early activation of auditory cortex (AC) and superior temporal gyrus (STG), together with parts of the thalamus (Th), putamen $(\mathrm{Pu})$, the mesial superior frontal gyrus (SFG) (extending from the supplementary motor area to the anterior cingulate cortex) and pre-motor (PreM) areas (dark green). Then, more extensive clusters in the SFG, pre-motor cortex, thalamus and putamen became active as well as the right medial frontal gyrus (MFG), insula (INS), the calcarine sulcus (CalS) and parts of the (mostly left) superior parietal lobe (SPL), precuneus, intraparietal sulcus and parietooccipital sulcus (light green to yellow). Subsequently, large parts of the (right) SPL, precuneus, medial and inferior occipital cortex and the parahippocampal gyrus (PHG) became active (orange). Last, parts of the right SPL, including intraparietal sulcus, and part of the right PHG were activated (dark orange to red). For an overview of all significantly activated regions see Chapter 2, Table 2.1. 


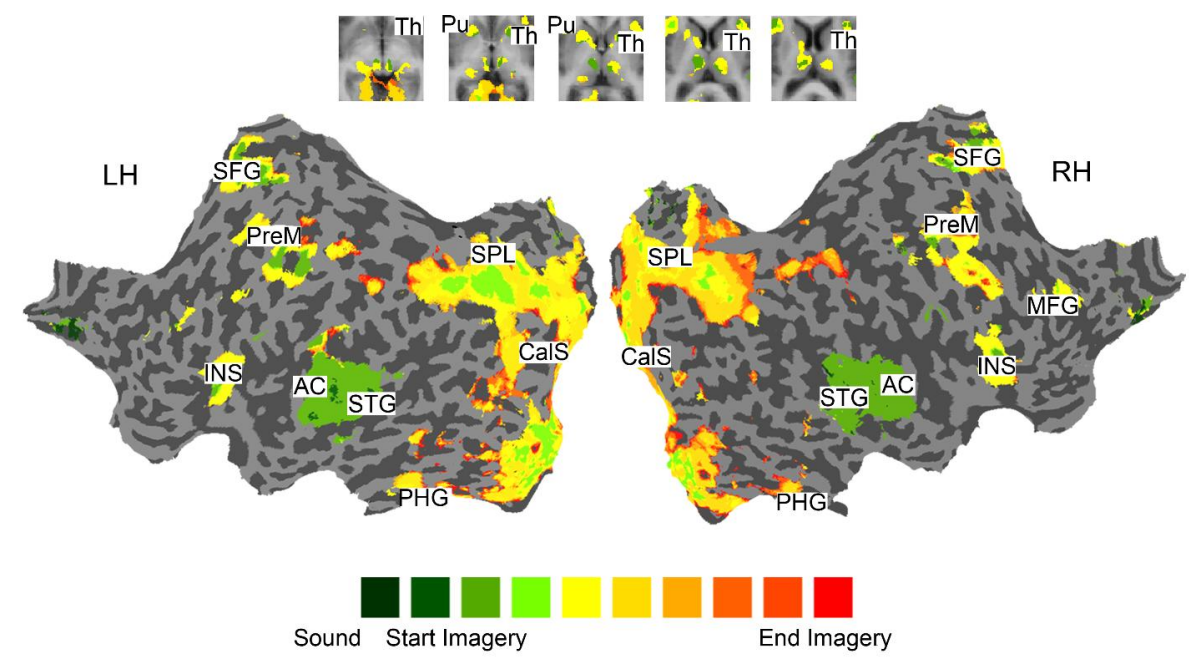

Figure 5.2 Multisubject (deconvolution) map during the mental imagery of scenes superimposed on a flattened representation of the cortical sheet. The voxels are colour coded according to their first significant beta value, representing the latency of BOLD activation during the auditory cue and mental imagery part of the trial. Top: Th $=$ thalamus, $\mathrm{Pu}=$ putamen. Left to right: $\mathrm{INS}=$ insula, $\mathrm{SFG}=$ mesial superior frontal gyrus, PreM $=$ premotor cortex, AC = auditory cortex, STG = superior temporal gyrus, PHG = parahippocampal gyrus, SPL = superior parietal lobule, CalS $=$ calcarine sulcus,$M F G=$ medial frontal gyrus .

\subsubsection{Granger Causality Mapping results}

To broaden our insight into the functional integration of the scene imagery network, a measure of effective connectivity, GCM analysis, was performed on the fMRI data. The results of the RFX group GCM are displayed in Figure 5.3 and 5.4. Only directed connections (dGCM), no instantaneous correlations, are displayed. This network shows left auditory cortex as an input region from which the incoming auditory cue is processed. From there, with the start of the mental imagery, influence is exerted to the mesial SFG, premotor cortex and calcarine sulcus. The mesial SFG can be seen as a central hub, with many connections to other nodes in the network. The mesial SFG exerts influence on the premotor cortex and calcarine sulcus, as well as the parahippocampal gyus, right middle frontal cortex and left and right parietal cortex. The right middle frontal gyrus also influences a number of regions: the calcarine sulcus, left parietal cortex and right parietal cortex. The calcarine sulcus only receives input from frontal regions and exerts influence over the left parietal cortex. The left and right parietal cortices receive input from frontal regions and exert influence on each other. Furthermore, the right parietal cortex projects back to the right auditory cortex. 


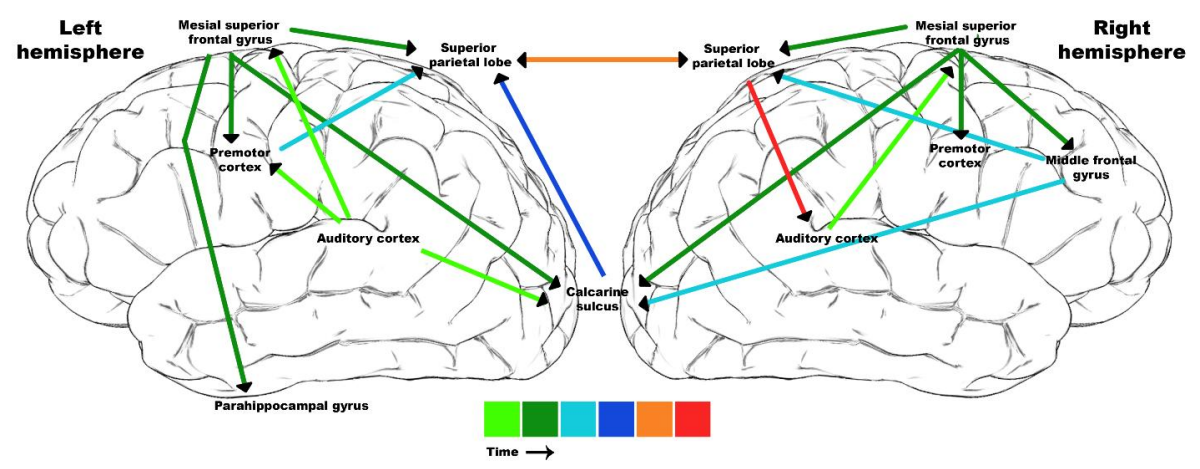

Figure 5.3 Results from a random effects Granger Causality Mapping group analysis. Directed influences from 9 regions of interest are displayed on representations of the cortex. The colour-coding indicates the timing during the task.

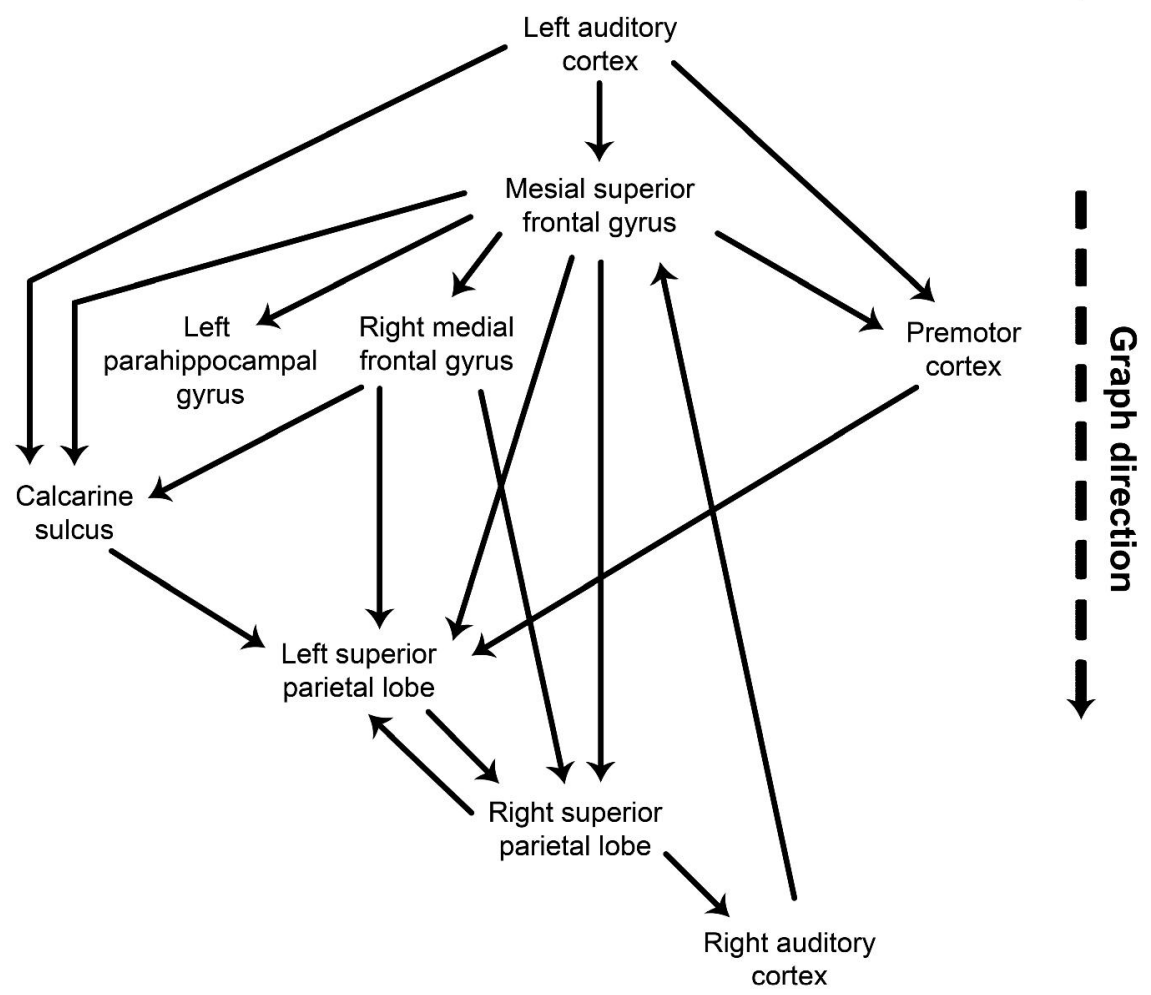

Figure 5.4 Results from a random effects Granger Causality Mapping group analysis. Directed influences from 9 regions of interest are displayed, organized according to their connections. 


\subsection{General discussion}

In this study we investigated the functional integration of a scene imagery network, by analyzing the effective connectivity of its regions with GCM. The results highlight the pattern of information flow through the cortex, which lies at the basis of the cascade of processing steps required to perform our task. This pattern indicates that information flows from the initial input in the auditory cortex, via frontal regions to regions in calcarine sulcus, parahippocampal gyrus and parietal cortex.

Together with knowledge gathered from our previous studies of the same mental imagery task (Chapters 2, 3 and 4), these GCM results allow defining a detailed neuro-anatomical model of scene imagery (see Figure 5.5). This model outlines the main nodes of the network, the possible processing flow of information through the network and the patterns of neuronal oscillations that may subserve the exchange of information. The main stages of the model are further discussed below.

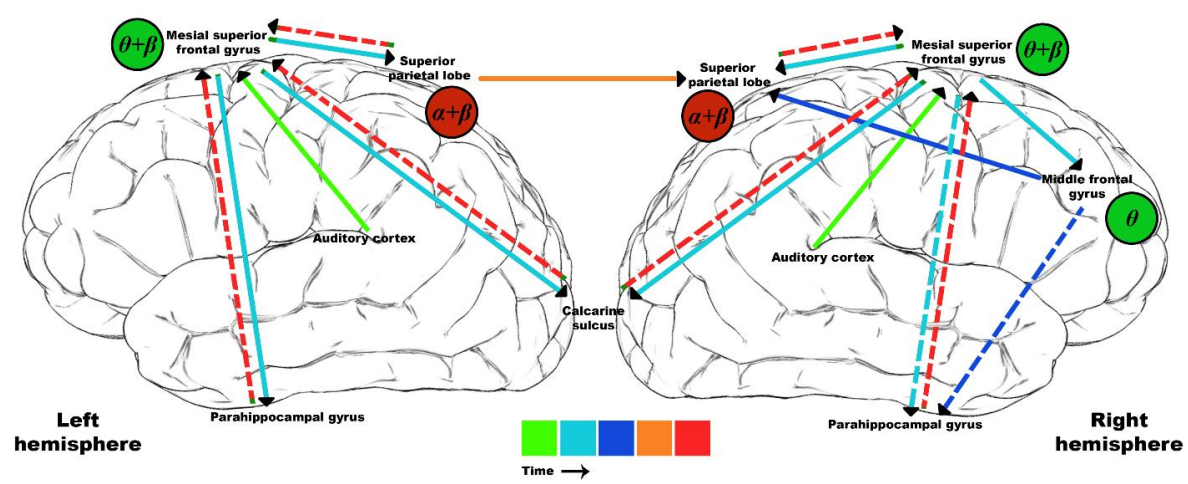

Figure 5.5 Neuro-anatomical model of scene imagery. Colours indicate timing during the task. Continuous line = confirmed by GCM analysis, dotted line = indicated by latency analysis. Alpha and beta de-synchronization is indicated in red circles, whilst theta and beta synchronization is indicated in green circles.

\subsubsection{Cue processing}

In the initial stage of the task, the auditory cue arrives in the cortex in bilateral early auditory cortex. From there more extensive processing of the cue is performed in superior temporal gyrus and sulcus (Figure 5.2). From auditory cortex information is send to the frontal cortex (mesial SFG and premotor cortex), where the process of mental 
imagery is initiated (Figure 5.3, 5.4 and 5.5). The GCM results furthermore indicate that auditory cortex and calcarine culcus are also directly connected. Although this result is not obvious, direct anatomical connections between early auditory cortex and early visual cortex have been reported (Falchier et al., 2002). This makes it not unlikely that in a task like ours, that requires detailed visualization of a scene, primary visual cortex is engaged early in mental image construction.

\subsubsection{The frontal relay station}

The results from the GCM analysis and our previous studies seem to indicate that one of the frontal regions, the mesial SFG, plays a central role as relay station during this task. It has many connections, both within frontal cortex, as well as to all remote regions, such as the calcarine sulcus, parahippocampal gyrus and bilateral superior parietal cortex. Previous research has indicated that the mesial SFG is involved in spatial and object feature integration and executive functioning (D'Esposito et al., 1995; Mitchell et al., 2000; Prabhakaran et al., 2000; Munk et al., 2002; Talati and Hirsch, 2005). This idea seemed to be confirmed by the EEG data of Chapter 3 which revealed a component with remarkable correspondence in location and behavioural relevance; the central anterior component. In fact, the midline frontal theta rhythm has been localized to anterior cingulate and medial prefrontal cortex in several studies (Gevins et al., 1997; Asada et al., 1999; Jensen and Tesche, 2002). This enables us to propose that the observed beta synchronization in mesial SFG reflects integration of information into a rehearsal set (Onton et al., 2005) and theta synchronization in mesial SFG is related to mental effort, attention and working memory maintenance and recall (Rugg and Venables, 1980; Gevins et al., 1997; Jensen and Tesche, 2002).

This integrative and essential role of the mesial SFG is further supported by the results of the prediction of reaction times in both the fMRI (Chapter 2) and EEG (Chapter 3) studies. Both datasets revealed the activity in this central anterior region to be predictive of task performance (see Figure 2.5 and 3.3), while other regions were not. Interestingly, mesial SFG in the fMRI data and the midline anterior IC in the EEG data showed opposite patterns in relation to task performance. A higher BOLD signal during mental imagery predicted slower reaction times, while stronger synchronization in the theta, alpha and beta bands during mental imagery predicted faster reaction times. One could theorize that during trials in which the participant found it difficult to imagine the scene, more interneurons were addressed to inhibit irrelevant input (Swadlow, 2003), 
leading to more oxygen consumption and higher BOLD signal, but less neuronal activity as measured by EEG (Buzsaki et al., 2007; Ekstrom, 2010).

As the effective connectivity results indicate, by connecting to both the ventral stream and the dorsal stream as well as early visual cortex (as indicated by the blue arrows in Figure 5.5), mesial SFG reaches all regions that are important for memory retrieval and construction of the image. Supportive roles seem to be played by the other frontal structures, the right middle frontal cortex and the premotor cortex. The right middle frontal cortex is reported in memory retrieval (Buckner et al., 1996; Tulving et al., 1996), a functional role which is supported by the EEG results of Chapter 3. These EEG results showed theta synchronization in a right lateralized anterior component, which is suggested to reflect memory retrieval and attention in the EEG working memory literature (Petsche et al., 1992; Bastiaansen et al., 2005; Deiber et al., 2007). This region is likely to also be connected to dorsal and ventral stream regions for memory retrieval (dark blue arrows Figure 5.4).

The reason why the mesial SFG is the central hub during our task, compared to the premotor cortex in Sack et al. (2008), is probably because our task involves both object and spatial processing. Since the premotor cortex is spatially oriented, it would be sufficient to rely on its connections to the parietal cortex during a dominantly spatial imagery task, whereas a task that requires equal activation of ventral and dorsal regions needs the mesial SFG, because it is also connected to lateral ventral cortex and early visual regions.

\subsubsection{Building the mental image}

Since the visual scene imagery task requires the imagination of objects and their spatial relations, both ventral (parahippocampal gyrus) and dorsal (superior parietal cortex, intra-parietal sulcus) stream are activated during this task. Surprisingly, these streams do not seem to exchange information between them, but rather are directed by the frontal regions. Actually, the only resolved input to the left parahippocampal gyrus comes from the relay station, the mesial SFG. The parahippocampal gyrus is responsible for retrieval of object content (Hayes et al., 2007; Prince et al., 2009).

The left superior parietal cortex does also receive input from the calcarine sulcus. Since the calcarine sulcus is directly mediated by the left auditory cortex, it is likely to be involved in the early stages of image build-up or visual (spatial) attention, and subsequently aiding the left superior parietal cortex. The left and right superior parietal cortexes are also interconnected. The results from the fMRI (Figure 2.3B) and 
EEG (Figure 3.2) studies indicated a latency difference between left (earlier) and right (later) parietal cortex. Speculatively, the connectivity pattern reveals some hints on the proposed functional distinction (Formisano et al., 2002; Sack et al., 2002; Sack et al., 2005) between left and right superior parietal cortex. Previous studies suggested that left superior parietal cortex is involved in construction of spatial relations, while the right performs visuospatial image inspection. Since the left parietal cortex receives input from the premotor cortex (deciphering a spatial environment) and the calcarine sulcus (possibly visual attention), a role in image construction is entirely feasible. Whereas to the role of the right parietal cortex we can only guess, as the GCM analysis is limited to the mental imagery part of the task and does not take the comparison of the target and the imagined scene into account. It is however possible that the participants inspect the spatial layout of the image while they are constructing it, but the GCM data (i.e. the connections from frontal cortex to right parietal cortex and its connections back to auditory cortex and left parietal cortex) do not give a decisive answer on this.

The remote regions in parietal, temporal and occipital cortex are likely to be involved in retrieval of content-specific information, guided by the mesial SFG which is orchestrating the whole process. These regions are therefore likely to project back to mesial SFG (see Figure 5.5) in order to update the visual mental scene. Feedback connections are often difficult to visualize, as the subtraction approach of the dGCM usually only shows the dominating direction of exchange.

\subsubsection{Issues for consideration}

When interpreting the Granger Causality data, another issue needs to be taken into account. Since Granger Causality relies on temporal precedence of one region before another, it is possible that hemodynamic differences between two regions cause an incorrect assignment of Granger Causality between these regions. Therefore, it is best to compare Granger Causality between two experimental conditions. Because our study consist of one condition versus baseline, it was not possible to perform such an analysis. However, the differences we found in connectivity between the left superior parietal cortex and the right superior parietal cortex indicate that our connectivity pattern is not purely generated by hemodynamic differences, as two largely homologue anatomical regions are not likely to differ in their neurovascular properties. Furthermore, some of the latency differences are confirmed by an EEG study with the same paradigm (Chapter 3). Moreover, a recent study by Schippers et al. (2011) took known hemodynamic differences between brain regions into account and revealed that a significant 
differential Granger causality is unlikely to be found without an actual underlying flow of information (Schippers et al., 2011). A more definite solution, and a goal for future research, is to perform GCM on these EEG data and compare connectivity networks. This is a feasible option, as most regions in the current network can be visualized with EEG.

\subsubsection{Conclusions}

In this study we showed it is possible to resolve the functional integration of a visual scene imagery network in a data-driven manner. We demonstrated direct connections from the input region (auditory cortex) to other regions in the network and revealed a central role of the mesial SFG. The mesial SFG exerted influence on modality-specific regions, such as the parahippocampal gyrus and the parietal cortex. Surprisingly, the calcarine sulcus also seemed to play an early role in image construction, as indicated by its direct input from auditory cortex and mesial SFG and its connection to left parietal cortex. Our results let us to propose a neuro-anatomical model of scene imagery in which by means of theta and beta synchronization the frontal regions seem to retrieve and integrate information, while orchestrating remote parietal regions for retrieval and representation of the spatial content of the scene and occipital-temporal regions for detailed visual representation of the objects. 
Alivisatos B, Petrides M (1997) Functional activation of the human brain during mental rotation. Neuropsychologia 35:111-118.

Asada H, Fukuda Y, Tsunoda S, Yamaguchi M, Tonoike M (1999) Frontal midline theta rhythms reflect alternative activation of prefrontal cortex and anterior cingulate cortex in humans. Neurosci Lett 274:29-32.

Bastiaansen MCM, Bastiaansen MCM, van der Linden M, Keurs Mt, Dijkstra T, Hagoort P (2005) Theta Responses are Involved in Lexical-Semantic Retrieval during Language Processing. Journal of Cognitive Neuroscience 17:530.

Buchel C, Friston KJ (1997) Modulation of connectivity in visual pathways by attention: cortical interactions evaluated with structural equation modelling and fMRI. Cereb Cortex 7:768778.

Buckner RL, Raichle ME, Miezin FM, Petersen SE (1996) Functional anatomic studies of memory retrieval for auditory words and visual pictures. Journal of Neuroscience 16:6219-6235.

Buzsaki G, Kaila K, Raichle M (2007) Inhibition and brain work. Neuron 56:771-783.

Cabeza R, Nyberg L (2000) Imaging cognition II: An empirical review of 275 PET and fMRI studies. J Cogn Neurosci 12:1-47.

D'Esposito M, Detre JA, Alsop DC, Shin RK, Atlas S, Grossman M (1995) The neural basis of the central executive system of working memory. Nature 378:279-281.

Deiber MP, Missonnier P, Bertrand O, Gold G, Fazio-Costa L, Ibanez V, Giannakopoulos P (2007) Distinction between perceptual and attentional processing in working memory tasks: a study of phase-locked and induced oscillatory brain dynamics. J Cogn Neurosci 19:158172.

Ekstrom A (2010) How and when the fMRI BOLD signal relates to underlying neural activity: the danger in dissociation. Brain Res Rev 62:233-244.

Falchier A, Clavagnier S, Barone P, Kennedy H (2002) Anatomical evidence of multimodal integration in primate striate cortex. J Neurosci 22:5749-5759.

Formisano E, Goebel R (2003) Tracking cognitive processes with functional MRI mental chronometry. Curr Opin Neurobiol 13:174-181.

Formisano E, Linden DE, Di Salle F, Trojano L, Esposito F, Sack AT, Grossi D, Zanella FE, Goebel R (2002) Tracking the mind's image in the brain I: time-resolved fMRI during visuospatial mental imagery. Neuron 35:185-194.

Friston KJ (1994) Functional and effective connectivity in neuroimaging: A synthesis. Human Brain Mapping 2:56.

Friston KJ, Harrison L, Penny W (2003) Dynamic causal modelling. Neuroimage 19:1273-1302.

Gevins A, Smith ME, McEvoy L, Yu D (1997) High-resolution EEG mapping of cortical activation related to working memory: effects of task difficulty, type of processing, and practice. Cerebral Cortex 7:374-385.

Goebel R, Esposito F, Formisano E (2006) Analysis of functional image analysis contest (FIAC) data with brainvoyager QX: From single-subject to cortically aligned group general linear model analysis and self-organizing group independent component analysis. Human Brain Mapping 27:392-401. 
Hayes SM, Nadel L, Ryan L (2007) The effect of scene context on episodic object recognition: parahippocampal cortex mediates memory encoding and retrieval success. Hippocampus 17:873-889.

Ishai A, Ungerleider LG, Haxby JV (2000) Distributed neural systems for the generation of visual images. Neuron 28:979-990.

Ishai A, Haxby JV, Ungerleider LG (2002) Visual imagery of famous faces: effects of memory and attention revealed by fMRI. Neuroimage 17:1729-1741.

Jensen O, Tesche CD (2002) Frontal theta activity in humans increases with memory load in a working memory task. European Journal of Neuroscience 15:1395-1399.

Knauff M, Kassubek J, Mulack T, Greenlee MW (2000) Cortical activation evoked by visual mental imagery as measured by fMRI. Neuroreport 11:3957-3962.

McIntosh AR, McIntosh AR, Gonzalez-Lima F (1994) Structural equation modeling and its application to network analysis in functional brain imaging. Human Brain Mapping 2:2.

Mechelli A, Price CJ, Friston KJ, Ishai A (2004) Where bottom-up meets top-down: neuronal interactions during perception and imagery. Cerebral Cortex 14:1256-1265.

Mellet E, Tzourio N, Crivello F, Joliot M, Denis M, Mazoyer B (1996) Functional anatomy of spatial mental imagery generated from verbal instructions. Journal of Neuroscience 16:6504-6512.

Mellet E, Tzourio-Mazoyer N, Bricogne S, Mazoyer B, Kosslyn SM, Denis M (2000) Functional anatomy of high-resolution visual mental imagery. Journal of Cognitive Neuroscience 12:98-109.

Menon RS, Kim SG (1999) Spatial and temporal limits in cognitive neuroimaging with fMRI. Trends Cogn Sci 3:207-216.

Mitchell KJ, Johnson MK, Raye CL, D'Esposito M (2000) fMRI evidence of age-related hippocampal dysfunction in feature binding in working memory. Cognitive Brain Research 10:197-206.

Munk MH, Linden DE, Muckli L, Lanfermann H, Zanella FE, Singer W, Goebel R (2002) Distributed cortical systems in visual short-term memory revealed by event-related functional magnetic resonance imaging. Cerebral Cortex 12:866-876.

O'Craven KM, Kanwisher N (2000) Mental imagery of faces and places activates corresponding stiimulus-specific brain regions. Journal of Cognitive Neuroscience 12:1013-1023.

Onton J, Delorme A, Makeig S (2005) Frontal midline EEG dynamics during working memory. Neuroimage 27:341-356.

Petsche H, Lacroix D, Lindner K, Rappelsberger P, Schmidt-Henrich E (1992) Thinking with images or thinking with language: a pilot EEG probability mapping study. International Journal of Psychophysiology 12:31-39.

Prabhakaran V, Narayanan K, Zhao Z, Gabrieli JD (2000) Integration of diverse information in working memory within the frontal lobe. Nature Neuroscience 3:85-90.

Prince SE, Dennis NA, Cabeza R (2009) Encoding and retrieving faces and places: distinguishing process- and stimulus-specific differences in brain activity. Neuropsychologia 47:22822289.

Repovs G, Baddeley A (2006) The multi-component model of working memory: explorations in experimental cognitive psychology. Neuroscience 139:5-21. 
Roebroeck A, Formisano E, Goebel R (2005) Mapping directed influence over the brain using Granger causality and fMRI. Neuroimage 25:230-242.

Rugg MD, Venables PH (1980) EEG correlates of the acquisition of high- and low-imagery words. Neuroscience Letters 16:67-70.

Sack AT, Camprodon JA, Pascual-Leone A, Goebel R (2005) The dynamics of interhemispheric compensatory processes in mental imagery. Science 308:702-704.

Sack AT, Jacobs C, De Martino F, Staeren N, Goebel R, Formisano E (2008) Dynamic premotor-toparietal interactions during spatial imagery. J Neurosci 28:8417-8429.

Sack AT, Sperling JM, Prvulovic D, Formisano E, Goebel R, Di Salle F, Dierks T, Linden DE (2002) Tracking the mind's image in the brain II: transcranial magnetic stimulation reveals parietal asymmetry in visuospatial imagery. Neuron 35:195-204.

Schippers MB, Renken R, Keysers C (2011) The effect of intra- and inter-subject variability of hemodynamic responses on group level Granger causality analyses. Neuroimage (epub ahead of print).

Stephan KE, Kasper L, Harrison LM, Daunizeau J, den Ouden HE, Breakspear M, Friston KJ (2008) Nonlinear dynamic causal models for fMRI. Neuroimage 42:649-662.

Swadlow HA (2003) Fast-spike interneurons and feedforward inhibition in awake sensory neocortex. Cereb Cortex 13:25-32.

Talairach J, Tournoux P (1988) Co-planar Stereotaxic Atlas of the Human Brain: 3-Dimensional Proportional System - an Approach to Cerebral Imaging. New York: Thieme Medical Publishers.

Talati A, Hirsch J (2005) Functional specialization within the medial frontal gyrus for perceptual go/no-go decisions based on "what," "when," and "where" related information: an fMRI study. Journal of Cognitive Neuroscience 17:981-993.

Trojano L, Trojano L, Linden DEJ, Formisano E, Goebel R, Sack AT, Di Salle F (2004) What clocks tell us about the neural correlates of spatial imagery. European Journal of Cognitive Psychology 16:653.

Tulving E, Markowitsch HJ, Craik FE, Habib R, Houle S (1996) Novelty and familiarity activations in PET studies of memory encoding and retrieval. Cerebral Cortex 6:71-79. 
Chapter 5

114 


\section{English summary}

In daily life we regularly and often unconsciously make use of mental imagery, for instance when we remember things, or think about a new idea or plan. This means that we can form a mental image very quickly and that we can also adapt it. Even though mental imagery seems very self-evident, a complex network of brain regions underlies this cognitive function.

Analogous to visual perception, visually imagined information is processed in two pathways: the ventral "what" and the dorsal "where" pathway. Modern brain imaging research discovered, using positron emission tomography (PET) and functional magnetic resonance imaging (fMRI), that the ventral pathway is active during the mental imagery of objects in a similar manner as during perception. This means that the imagination of objects within certain categories elicits brain activity in specific parts of the ventral temporal cortex. On the other hand, it has been demonstrated that during the imagination of spatial information the dorsal pathway in the parietal cortex is activated. Imagined information is thus processed in a similar manner in two pathways in the brain as perceived information. However, perception and mental imagery are evidently not the same. After all, we do not confuse reality with our thoughts. When we use mental imagery, several regions in the frontal cortex of our brain are also activated. These regions help in recalling memories and executing mental operations.

All regions in this distributed mental imagery network work closely together and communicate with each other. Both on the local neuronal level, as on the level of brain regions, communication takes place through oscillations. Synchronized oscillations of a large population of neurons can be measured on the skull using electroencephalography (EEG). During mental imagery, oscillations in the theta, alpha and gamma frequency bands dominate. Theta synchronization takes place in the frontal parts of the brain during working memory operations. Theta synchronization is often accompanied by gamma oscillations in the dorsal or ventral pathway during the imagination of locations or objects, respectively. Mental imagery is supported by alpha (de-)synchronization. Synchronization in the alpha frequency is often considered to reflect inhibition in task-irrelevant regions, while de-synchronization of alpha reflects cognitive involvement. 
In this dissertation we have studied the neural basis of mental imagery with a series of fMRI, EEG and combined EEG-fMRI studies. By using progressive methods we have managed to add thus far missing information to the mental imagery literature. Instead of focusing on one of the subsystems of mental imagery such as object or spatial imagination, or working memory, we have investigated these systems within one task. By using this task systematically in studies with various measurement techniques, we were able to compare and validate information on the topography, timing and relationships between regions in the mental imagery network. With each chapter we resolved another piece of the puzzle, by investigating the different attributes of the network, and merged these pieces into a neuro-anatomical model of mental imagery in the final chapter.

In Chapter 2 we have used fMRI to investigate what brain regions are active at which point during a task that requires both object and spatial imagination. We found a large functional network with active regions in a.o. frontal cortex, the "what" and "where" pathways and the early visual cortex. It turned out that within this network the frontal regions are active early during the task. One of these frontal regions, which is located at the midline of the brain (the mesial superior frontal gyrus) is of considerable significance, since its activity correlates with task performance. This region seems to arrange the integration of information from the parietal regions, where, as the results indicated, the spatial design of the location is represented, and from the temporal regions where the details of the objects are depicted. Subsequently, we have investigated which brain oscillations underlie the imagery of objects and their lay-out in an EEG study with the same task in Chapter 3. We found that the recollection of (spatial) information from memory is supported by theta oscillations in a right frontal region, together with beta oscillations in bilateral parietal regions. Theta and beta oscillations, in a mid-frontal region, seem to lie at the foundation of integrating information (objects and space) into one mental scene. The total imagery process seems to be supported by alpha de-synchronization in occipito-parietal regions, which reflect attention or inhibition of non-relevant areas. To connect the results of these two chapters, we have performed a simultaneous EEG-fMRI study, as described in Chapter 4. In this study we applied a new technique, Relevance Vector Machine regression, to predict EEG patterns from fMRI data. The results of the prediction of oscillations in the alpha and beta bands in a midfrontal component during the mental imagery task indicated a coupling with the mesial superior frontal gyrus within a network of regions such as the intraparietal sulcus and the ventral visual regions. From this we can conclude that theta and beta oscillations in the mid-frontal component, as reported in chapter 3, most likely underlie the integrative role of the mesial superior gyrus, as found in chapter 2 . We have further investigated the 
communication between regions in the mental imagery network with a connectivity analysis in Chapter 5. Through means of a data driven analysis technique, Granger Causality Mapping, we found that information is sent from the auditory regions to the frontal regions and the early visual cortex. One of the frontal regions, the mesial superior frontal gyrus, has many connections to other frontal regions, the early visual cortex and the "what" and "where" regions, which confirms its central and integrative role, as proposed in previous chapters. The parietal "where" regions receive information late in the hierarchy and, surprisingly, we have found no direct communication with the "what" regions in the temporal cortex. Instead, the mental scene seems to be formed via the frontal cortex.

Integrating the knowledge gathered from the various studies, we propose a neuro-anatomical model of visual mental imagery: the cue is processed in (depending on the modality) the auditory cortex. Subsequently, the frontal cortex is exerted to start the mental imagery process. By means of theta and beta synchronization the stored images are recollected and communication with distant regions in visual, temporal and parietal cortex is initiated. In visual and temporal cortex the (details of the) objects are recollected and represented, while in parietal cortex through beta desynchronization the spatial layout of the locations is processed. Simultaneously, information is updated and the visual scene is formed with help of the mesial superior frontal gyrus.

We can conclude that the studies in this dissertation contributed to a new model of the brain dynamics that underlie mental imagery, in which topographical, spectro-temporal and connective properties are integrated. 
118 


\section{Nederlandse samenvatting}

In het dagelijks leven maken we heel regelmatig en vaak onbewust gebruik van ons voorstellingsvermogen, bijvoorbeeld wanneer we ons dingen herinneren, of nadenken over een nieuw idee of plan. Dit betekent dat we heel snel een mentaal beeld kunnen vormen en deze ook kunnen aanpassen. Ook al lijkt ons voorstellingsvermogen heel vanzelfsprekend, er ligt een complex netwerk van hersengebieden ten grondslag aan deze cognitieve functie.

In overeenstemming met de visuele waarneming, wordt visueel ingebeelde informatie verwerkt in twee paden: het ventrale "wat" en het dorsale "waar" pad. Modern hersenonderzoek dat gebruik maakt van positronemissietomografie (PET) en functionele kernspintomografie (functional magnetic resonance imaging; fMRI) heeft ontdekt dat tijdens de mentale inbeelding van objecten het ventrale pad actief is op een vergelijkbare manier als tijdens de waarneming. De inbeelding van objecten binnen bepaalde categorieën veroorzaakt hersenactiviteit in specifieke delen van de temporale cortex. Aan de andere kant is aangetoond dat tijdens het voorstellen van ruimtelijke informatie het dorsale pad in de pariëtaal cortex geactiveerd wordt. Ingebeelde informatie wordt dus op een vergelijkbare manier in twee hersenpaden verwerkt als waargenomen informatie. Natuurlijk zijn waarneming en inbeelding niet hetzelfde, we verwarren de werkelijkheid immers niet met onze gedachten. Wanneer we ons dingen voorstellen zijn ook een aantal gebieden in de frontale cortex van ons brein actief. Deze gebieden helpen bij het ophalen van herinneringen en het uitvoeren van mentale bewerkingen.

Al deze gebieden in dit gedistribueerde netwerk werken met elkaar samen en communiceren met elkaar. Zowel op lokaal neuronaal niveau, als op het niveau van hersengebieden vindt communicatie plaats door middel van oscillaties. Gesynchroniseerde oscillaties van een grote populatie neuronen kunnen gemeten worden op de schedel met behulp van elektro-encefalografie (EEG). Bij het gebruik van het voorstellingsvermogen overheersen oscillaties in de theta, alfa en gamma frequentie banden. Theta synchronisatie in frontale delen van de hersenen vindt plaats tijdens het gebruik van werkgeheugen. Theta synchronisatie gaat vaak samen met gamma synchronisatie in het dorsale of ventrale pad tijdens het voorstellen van locaties of objecten. Het mentaal voorstellingsvermogen wordt ondersteund door alfa 
(de)synchronisatie. Synchronisatie in de alfa frequentie wordt vaak gezien als de inhibitie van taak irrelevante gebieden, terwijl de desynchronisatie van alfa cognitieve betrokkenheid reflecteert.

In dit proefschrift hebben we door middel van een serie van fMRI, EEG en gecombineerde EEG-fMRI studies de neurale basis van het voorstellingsvermogen onderzocht. Door het gebruik van vooruitstrevende methodes, zijn we erin geslaagd om tot nu toe ontbrekende informatie toe te voegen aan de literatuur van mentaal voorstellingsvermogen. In het bijzonder hebben we in plaats van ons te richten op één van de subsystemen van het voorstellingsvermogen, zoals object of ruimtelijke inbeelding, of het gebruik van het werkgeheugen, deze systemen onderzocht binnen één taak. Door deze taak systematisch te gebruiken in studies met verschillende meetinstrumenten zijn we in staat geweest om informatie over de topografie, tijdsvolgorde en relaties tussen gebieden in het voorstellingsvermogen netwerk met elkaar te vergelijken en valideren. Ieder hoofdstuk draagt bij tot een stukje van de puzzel, waarbij een ander kenmerk van het netwerk onderzocht wordt, samenvloeiend tot een neuro-anatomisch model van het voorstellingsvermogen in het laatste hoofdstuk.

In hoofdstuk 2 hebben we fMRI gebruikt om te onderzoeken welke hersengebieden wanneer actief zijn tijdens een taak die object en ruimtelijk voorstellingsvermogen vereist. We vonden een groot functioneel netwerk waarbij o.a. gebieden in de frontale cortex, in de "wat" en "waar" paden en in de vroege visuele cortex actief waren. Binnen dit netwerk bleek dat de frontale gebieden vroeg tijdens de taak actief zijn. Eén van deze frontale gebieden, welke tegen de middenlijn van het brein aanligt (de "mesial superior frontal gyrus"), is van aanzienlijk belang daar de activiteit in dit gebied samenhing met de prestatie op de taak. Dit gebied regelt vermoedelijk de integratie van informatie uit de pariëtale gebieden waar, zoals de resultaten aangaven, de ruimtelijke vormgeving van de locatie weergegeven wordt en uit de temporele gebieden waar de details van de visuele objecten ingevuld worden. Vervolgens hebben we in hoofdstuk 3 met behulp van dezelfde taak in een EEG studie onderzocht welke hersenoscillaties het voorstellen van objecten en hun lay-out onderliggen. We vonden dat het ophalen uit het geheugen van (ruimtelijke) informatie gesteund wordt door theta oscillaties in een rechter frontaal gebied, samen met de desynchronizatie van beta oscillaties in bilateraal pariëtaal gebieden. Theta en beta oscillaties in een midden frontaal gebied lijken ten grondslag te liggen aan de integratie van informatie (objecten en ruimte) tot één mentale locatie. Het gehele voorstellingsproces wordt ondersteund door alfa desynchronizatie in de occipito-pariëtale gebieden, welke aandacht of de inhibitie van gebieden die niet relevant voor de taak zijn reflecteren. Om de resultaten van deze twee hoofdstukken met elkaar te verbinden hebben we een gelijktijdige EEG- 
fMRI studie uitgevoerd, zoals beschreven in hoofdstuk 4. In deze studie maakten we gebruik van een nieuwe techniek, "Relevance Vector Machine" regressie, om EEG patronen te voorspellen vanuit fMRI data. De resultaten van de voorspelling van oscillaties in de alfa en beta band in een midden frontale EEG component tijdens de mentale voorstellingstaak duidden op een koppeling met de "mesial superior frontal gyrus" binnen een netwerk van gebieden zoals de "intraparietal sulcus" en de ventrale visuele gebieden. Hierdoor kunnen we concluderen dat theta en beta oscillaties in de midden frontale EEG component, zoals gevonden in hoofdstuk 3, zeer waarschijnlijk ten grondslag liggen aan de de integratieve rol van "mesial superior frontal gyrus" zoals gevonden in hoofdstuk 2. We hebben de communicatie tussen gebieden in het mentale voorstellingsnetwerk verder onderzocht met een connectiviteitsanalyse in hoofdstuk 5 . Met behulp van een door de data aangedreven analyse techniek, "Granger Causality Mapping", vonden we dat informatie vanuit de auditieve cortex naar de frontale gebieden en de vroege visuele cortex gestuurd wordt. De "mesial superior frontal gyrus" blijkt vele connecties te hebben naar andere frontale gebieden, de vroege visuele cortex en de "wat" en "waar" gebieden, wat de centrale en integratieve rol van dit gebied, zoals voorgesteld in vorige hoofdstukken, bevestigd. De pariëtale "waar" gebieden krijgen laat in de hiërarchie informatie binnen en, verrassend genoeg, is er geen directe communicatie met de "wat" gebieden in de temporale cortex gevonden. In plaats daarvan lijkt de mentale voorstelling via de frontale cortex gevormd te worden.

De opgedane kennis uit de diverse studies integrerend, stellen we een neuroanatomisch model van het visuele mentale voorstellingsvermogen voor: De cue wordt verwerkt in de (afhankelijk van de modaliteit) auditieve cortex. Vervolgens wordt de frontale cortex aangewend om het voorstellingsproces te starten. Door middel van theta en beta synchronisatie worden de opgeslagen beelden herinnerd en communicatie met de verder gelegen gebieden in de visuele cortex, de temporale cortex en de pariëtaal cortex gestart. In de visuele en temporale cortex worden de (details van de) objecten opgehaald en gerepresenteerd, terwijl in de pariëtaal cortex door middel van beta desynchronizatie de ruimtelijke layout van de locatie wordt verwerkt. Tegelijkertijd wordt met hulp van de "mesial superior frontal gyrus" de informatie bijgewerkt en de visuele locatie gevormd.

Concluderend kunnen we dus stellen dat de studies van dit proefschrift hebben bijgedragen tot het vormen van een nieuw model van de breinprocessen die ons voorstellingsvermogen onderliggen, waarbij topografische, temporele en interacteve kenmerken geïntegreerd zijn. 
122 


\section{Acknowledgements}

At the end of my $\mathrm{PhD}$ and this booklet I want to thank all the people that have supported, encouraged and helped me with their expertise and friendship. I've tried to be as concise as possible with my thesis, but I'm afraid I'm going a little overboard with the thank-you's. I've so much to be grateful for.

Let's start at the beginning, when I chose to do my internship with Elia - a lucky strike as it turned out. Elia, thank you for your supervision during the past years. You have taught me to do solid research and to combine my psychology background with loads of new methods. The years of my PhD have not been easy years for you and Milene, but I am really happy that everything looks better now with Luca. You are my great example of how to be a researcher, and I'm sure you'll keep inspiring everyone you supervise and help. Francesco, I want to thank you for giving me the opportunity to do this $\mathrm{PhD}$, starting it in beautiful Italy! Thank you for giving me the freedom and independence to execute my research as I wanted.

Lucia and Ilaria, thank you for adopting me into your homes in Pisa, I wouldn't have made it without you. Nicola, thank you for making me smile with your visits. Adriana and Fabrizio, also the both of you have cheered me up when I was far away from my loved ones. It was always a joy when you came to visit from Napoli and collaborating with me on work. Thank you for your help and support.

Another five people without whom all this work wouldn't have happened: dad, mom, Martin, Joanneke and Hein! Mom and dad, thank you for always supporting me in the idea that I could do anything and become anyone, and - equally important - that even if something didn't work out, it's ok as long as I tried. And of course I'm also very grateful for your financial support during my studies and PhD. Brother, Ama, Marinde, little sis and bro, I am really happy that you are my family! Hopefully we'll have many more nice weekends and holidays together!

I could have never imagined finding such a fantastic work place, with such great friends and colleagues! It has been a place to learn, but also a place to socialize and have fun. I have all of you to thank, both on work-wise advice and partying it up! 
Marin, my thesis buddy and friend :) I think we have spent more time together during the past 7 years than with anyone else! People even started seeing us as one person at some point! ;-) I really really appreciate all the chats over tea that we've had, both on work and all other issues. Thank you for being my friend. Mariekje, your down-to-earth outlook on life and your incredible motivation for work have both inspired me to work harder and distract you a little more ;-). Even when you were far away in the US, we kept close (we should do the same for Cambridge!). I'm so happy to have you around. Rico, we connected instantly, and through times of closeness and wanting to shoot each other ;-), I'm happy to still call you my good friend. Also on a working level I have to thank you for always being ready to help me out and for your collaboration on Chapter 3. I hope we'll always stay in contact bello. Gianca! I have really appreciated our friendship during our early years of working for Maastricht University - two slightly depressed dark souls. Happy to see life is looking brighter now :). I love how one simple question can make you produce half an hour of explanation and two hours of code. Thank you for all your help! Michael, what a wonderful combination between a gossip girl and big strong hunk you are :). You know I always tease you, so how could I stop now. My dear friend, thank you for the wonderful evenings of drinking whiskey, while chatting about sex, work, feelings, philosophy and everything in between.

Betty, Judith and Joel, I probably owe you an apology for all the chatting Marin and me have done in the data processing room, back when all of our computers were not able to handle motion correction :). Thank you for always being so kind. Valerie and Bernadette, you two have been (unknowingly probably ;-)) my examples and inspiration of how to have it all, to be a good researcher, to have a family and staying incredibly cool at the same time. Rob, thank you for giving MAA the opportunity to find our research spirit :). Lars R., thank you for your help on making auditory stimuli. Alex, thank you for helping with the design of the experiments and teaching me a thing or two on how to write slightly juicier papers. David, Lars, Amanda, Axel and all the others at the yearly imagery meetings, thank you for your feedback on my work. I'll never forget our one attempt to discover "Schmitten nightlife" in the local hiphop disco.

Nick, Martin and Francesco, thanks for coming up with an inexhaustible amount of lunch conversation topics, or wait.. maybe it was just a cycle of 10 topics that kept returning? :P Nici, thanks for reminding me that maybe sometimes we take science a little too seriously and that psychology is cool too (in our failure to always understand as much as the engineers, physicists and mathematicians around us). Dries, Gonny, Anniek, Teresa, Jeanette and Petra, thanks for always being willing to participate in a crazy night out or another movie project. Noelleke, thank you for your great sense of humour. Peter, what fun have we had in organizing the international dinner parties! Even cleaning up is wonderful with some nice port and cheese. You're such a great guy, 
I have really appreciated all your advice, chats and dances. Anke, Michelle, Lars, Job, Tom, Roberta, Britta and Els, thank you for the nice lunch chats and crazy conference experiences! My other roomy and next-door neighbours, Nina, Christianne, Julien, Joost, Pim, Hester and Sven, thanks for all your friendliness. Mehrdad, Kamil and Joao, I'm so happy to have you as the new additions to our department! You guys are great :). I'm happy to see some party spirit return to the department. Henk, Vince, Anton, thank you guys for (unknowingly) brightening up my day once every while with a sarcastic comment or joke. Alard, you are always willing to help out and explain, that's a wonderful characteristic. Thank you for your help and the nice parties (and horrible hangovers) we have thrown together.

Annemie, Riny and Christl, thank you for always helping me (and everyone else) out with all administrative business. Maurice, thanks for solving all my computer mysteries.

Finally, a big thank you to all my other colleagues, even those I have not gotten to know so well over the years - I believe we make a wonderful department all together!

Of course I have received a lot of support outside of work. My dear friends, thank you!!

First and foremost, Lauran. You are the one I have to thank most of all people in this note. Your everlasting support and sweetness have helped me through so much. Even when I was far away in Pisa, you never gave up on us. The years of my $\mathrm{PhD}$ also have been hard years for you, your illness was the scariest thing I've experienced. I'm so happy to still have you around as my friend, you're my light, thank you poeksk.

Annepan! Knowing each other for over 10 years, you understand me so well. Thank you for all the crazy stuff we've done together and that I am always welcome to call you or drop by at times the world becomes a bit too much. Thanks for all your support, you're wonderful. Flora! Ha, I wonder how many emails and messages we have exchanged over the years! Sometimes 10 times a day we would update each other on what was going on :). Also you I have to thank for all your support over the years and listening to my endless whining ;-). Marije, without you I maybe would have never done my $\mathrm{PhD}$ ! I'm so grateful for dragging me along to meet Rob, leading to our first experiences in research as student assistants. For spontaneously making sure we went to visit Bernardo in Mexico, a life-changing experience for me. And for picking cognitive neuroscience as a master :). So I think that in general your spontaneous nature, your decisiveness and great attitude have been a great help for me. Thank you! MAA forever ;-) Wendy, thanks for being such a nice neighbour! We are so similar in our love for everything foreign ;-). Thank you for all the long conversations we've had. Baukelien, I'm really happy that we are still friends after 13 years. I'm glad to still have one partner in crime who likes to 
choose her own path in life, rather than following "what we are supposed to do". Kortom, kipjes ftw!!!

Except for kipjes, there are also haantjes ofcourse! Timski, Chunniebun, Murx \& Japs thank you for your friendships over the years! Chun, I hope to have many more coffeelovers /bagels\&beans afternoons! Rica, you are the most wonderful "husband" I could have ever wished for. Thanks for your great sense of humour :-). Kenny, who would have thought that after that 5 minute conversation between Fontainebleau's boulders we would keep mailing each other so much for the past year and a half? I'm real glad to share my love for music, photography, climbing and silly humour with you. Guillaume, je t'adore. You are one of these rare special people that have magically turned up in my life. Thank you for your kindness, all our conversations, the wonderful conferences, and for being such a great example of an honourable and modest person and researcher. You make me endlessly smile.

Another blessing in my (PhD) life, is the crrrrazy Delft crew coming into my life! Adam, David, Marie-Eve, Ahmad, Kasra, Asia, Alex, Bogdan, Vladimir, Ouiam, Nadera, Mariana, Deborah, Zohar and everyone else, thank you so much for all the fun times, political and scientific discussions, parties, beers, holidays, climbs, dances and midnight conversations together. It means so much to me. You've taught me neuroscience is cool, dolphins are evil and that cultural differences don't matter at all!! Let's continue that! Dimaatje! "Wil je achterop zitten?" Haha, I'd never forget the brilliant Dutch you've learned. Thank you for feeding me cakes, food and tea at your kitchen table, while having more, or less, serious chats. You're great! And who would have thought all of that would have come from this one conversation on the use of little flying robots at a science party in Nemo? Filip, I'm so happy you have entered my life. You have taught me to chill more and plan less and to accept life as it comes. I admire your ability to be open to everyone, making friends everywhere you go. Thank you for pulling me out of my shell. I hope we'll remain in contact, wherever in the world we go, and have some more dances together.

Lalo, how I would have liked to email you now, proudly telling you I have finished, to hear you call me doctor Aline Wilhelmina de Borst. I miss you my dear crazy one. I'll try to remember your "don't worry". Thank you for giving me confidence. I imagine you somewhere, united with your little girl, smiling back at us.

The list of people who I'm grateful for is endless and more thank you's didn't fit onto these pages. The downfall of thanking everyone by name is that you are bound to forget someone, so just tell me how I can make it up to you ;-) 


\section{Curriculum Vitae}

Aline de Borst was born in Deventer, the Netherlands, on the 28th of June 1982. After completing high school (VWO) in 2000, she moved to Maastricht to study Psychology at Maastricht University. From the first year on, she started working as a research assistant next to her studies. In the five years of her studies she completed two masters (August 2005); one in cognitive ergonomics and one in cognitive neuroscience, and assisted in several studies on e.g. public health campaigns and placebo effects on memory. Her interest in cognitive neuroscience was stimulated by participating in (writing) projects on the influence of fMRI scanner noise and body position on cognitive tasks, object recognition and Brain Computer Interfaces. With her internship and master thesis work on "Exploring the imagery network with time-resolved functional MRI during the mental representation of scenes" supervised by dr. Elia Formisano, the fundaments were laid for a PhD on the same topic. She started her PhD with prof. Francesco di Salle in Italy, at the University of Pisa and completed the work in the Netherlands at Maastricht University.

\section{Publications, presentations \& grants}

- $\quad$ de Borst, A.W., Sack, A.T., Formisano, E., Roebroeck, A. (2011). Effective connectivity of the mental imagery network (in preparation).

- de Borst, A.W., Sack, A.T., Jansma, B.M., Esposito, F., De Martino, F., Valente, G., Roebroeck, A., Di Salle, F., Goebel, R., Formisano, E. (2011). Integration of "what" and "where" in frontal cortex during visual imagery of scenes (submitted).

- De Martino, F., de Borst, A.W., Valente, G., Goebel, R., Formisano, E. (2010) Predicting EEG single trial responses with simultaneous fMRI and Relevance Vector Machine regression. NeuroImage, Aug 4 (epub ahead of print).

- De Martino, F., Valente, G., de Borst, A.W., Esposito, F., Roebroeck, A., Goebel, R., Formisano, E. (2010). Multimodal imaging: an evaluation of univariate and multivariate methods for simultaneous EEG/fMRI. Magnetic Resonance Imaging, 28, 1104-12.

- de Borst, A.W., Sack, A.T., Jansma, B.M., Esposito, F., De Martino, F., Valente, G., Roebroeck, A., Di Salle, F., Goebel, R., Formisano, E. Spatial topography (fMRI) and spectro-temporal brain dynamics (EEG) during mental imagery of scenes. 15th Annual meeting of the organization for Human Brain Mapping, San Francisco, USA, June 2009. (poster)

- de Borst, A.W., Sack, A.T., Esposito, F., Goebel, R., Formisano, E. Timeresolved fMRI during imagery of complex scenes. 12th Annual meeting of the organization for Human Brain Mapping, Florence, Italy, June 2006. (poster)

- OHBM Travel Grant 2006. 12th Annual meeting of the organization for Human Brain Mapping, Florence, Italy, June, 2006. 